\title{
Role of WRB protein in cardiac function
}

\author{
PhD Thesis
}

Dissertation for the award of the degree

"Doctor of Philosophy"

Division of Mathematics and Natural Sciences

of the Georg-August-Universität Göttingen

within the Göttingen Graduate School for Neurosciences, Biophysics and

Molecular Biosciences (GGNB)

of the Georg-August University School of Science (GAUSS)

Submitted by

Jhon Erick Rivera Monroy

Cachipay, Colombia

Göttingen, March 2017 


\section{Members of the Thesis Committee and Examination Board:}

Supervisor, reviewer

Thesis committee, reviewer:

Thesis committee, reviewer:

Reviewer:

Reviewer:

Reviewer:

\author{
Prof. Dr. Blanche Schwappach \\ Department of Molecular Biology, \\ University Medical Center Göttingen \\ Max Planck Institute for Biophysical \\ Chemistry
}

Prof. Dr. Michael Meinecke

Department of Biochemistry II, University of Göttingen

European Neuroscience Institute

Dr. Hans Dieter Schmitt

Max Planck Institute for Biophysical

Chemistry

\section{Prof. Dr. Michael Thumm}

Department of Cellular biochemistry University Medical Center Göttingen

\section{Prof. Dr. Ralph Kehlenbach}

Department of Molecular Biology, University Medical Center Göttingen

Prof. Dr. Ivo Feussner

Department of Plant biochemistry University of Göttingen

Date of oral examination: May $18^{\text {th }}, 2017$ 


\section{Affidavit}

I hereby declare that I prepared the PhD thesis "Role of WRB protein in cardiac function" on my own and with no other sources and aids than quoted.

Göttingen, March 31 $1^{\text {st }}, 2017$

Jhon Erick Rivera Monroy 
Adam and Eve were black? The human adventure in the world began in Africa. From there, our ancestors set out to conquer the planet. Many paths led them to many destinies, and the sun took care of handing out colors from the palette.

Now the rainbow of the earth is more colorful than the rainbow of the sky. But we are all emigrants from Africa. Even the whitest of whites comes from Africa. Maybe we refuse to acknowledge our common origins because racism causes amnesia, or because we find it unbelievable that in those days long past the entire world was our kingdom, an immense map without borders, and our legs were the only passport required.

Eduardo Galeano. Mirrors: stories of almost everyone. 


\section{Acknowledgements}

First of all, I would like to thank Prof. Blanche Schwappach for giving me the opportunity to come to her lab to do what I love. I cannot express with words my gratitude for her tireless supervision, for the help, for the suggestions and passionate discussions, which have deeply contributed not only to my $\mathrm{PhD}$ but also to my professional and personal development. I had a lot of fun in my daily life as a PhD student and every moment I spent in this adventure was thanks to her.

Special thanks to Dr. Fabio Vilardi for his role as co-supervisor during my studies and for helping me particularly at the beginning of this journey. Thanks a lot for the ideas, discussions, suggestions that made my $\mathrm{PhD}$ studies possible.

I want to thank Prof. Michael Meinecke and Dr. Hans Dieter Schmitt, members of my Thesis Committee, for guiding me through my $\mathrm{PhD}$ and always having nice suggestions regarding my project.

I am grateful to Dr. Eric Arakel for the fantastic advice and pertinent comments on my work, that many times resulted in good results. I am very thankful to Eric for his friendship and for always being there not only for me but also all the other students of the lab.

I would like to thank Kirsten Unthan-Fechner who assisted me during the isolation of cardiomyocytes and was responsible for the animal breeding and genotyping. She always did a great job and made a very important contribution to the present work.

I want to thank all the present and past members of the Department of Molecular Biology for the great working environment. Many thanks to my friends Tobi, Javi, Markus, Jimena and Julia for the laughs and the coffee 
breaks, which many times catalysed excellent ideas and kept my motivation high and made me feel at home.

Also, I want to express my gratitude to my alma mater, the National University of Colombia for the excellent professors I had the fortune to meet. Particularly, I am very grateful to Prof. Marcela Camacho for the guidance, training and support I got in her biophysics lab during my bachelors and masters studies.

I cannot express with words how thankful I am to Laura, my wife, for her endless love, support, and for always being by my side in this adventure. She is the most precious piece of Colombia that came with me to Germany and I dedicate this thesis to her.

I want to thank my parents Gloria and Edgar and my siblings, Zuly and Alex. I owe them everything and I really hope that I have met all their expectations.

For its financial support, I am thankful to the ITN "TAMPting" network, funded by the People Programme (Marie Curie Actions) of the European Union's Seventh Framework Programme (FP7/2007-2013/) under the Research Executive Agency [grant number 607072]. I also thank all the professors, postdocs, $\mathrm{PhD}$ students and companies that were part of this network for their valuable comments concerning my project. 


\section{Table of Contents}

Abstract............................................................................................ 7

Authors contributions to the listed publications ................................8

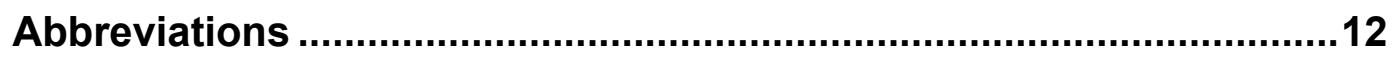

1. Introduction.............................................................................. 13

Biogenesis of integral membrane proteins of the secretory pathway....13

Pathways for the insertion of ER membrane proteins ......................14

The TRC40/Get3 pathway ................................................... 17

Alternative roles of the TRC40/Get3 ATPases ...............................21

Redundancy of the insertion pathways .......................................24

Animal models of the TRC40-pathway components .........................25

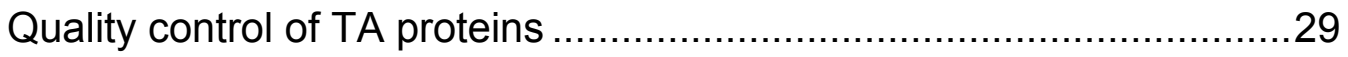

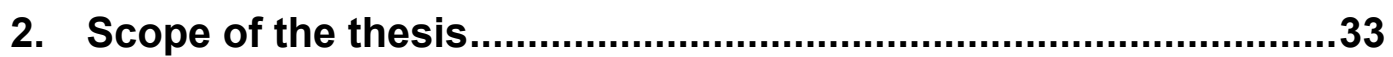

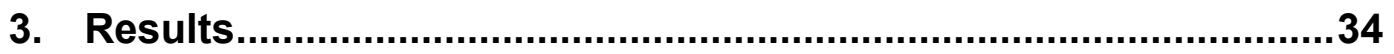

Manuscript 1: Emery-Dreifuss muscular dystrophy mutations impair TRC40-mediated targeting of emerin to the inner nuclear membrane. .34 Manuscript 2: Mice lacking WRB reveal differential biogenesis requirements of tail-anchored proteins in vivo................................50

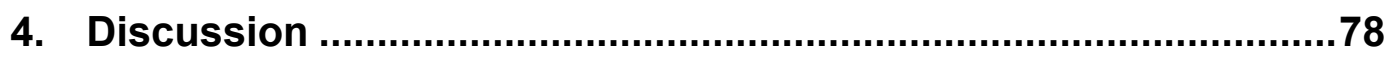

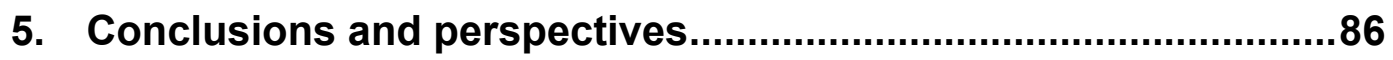

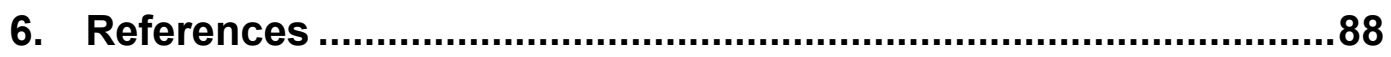




\section{Abstract}

The mammalian TRC40 system is a conserved pathway responsible for the post-translational targeting of tail-anchored proteins, which are characterized by a single transmembrane domain at the C-terminus and an N-terminus that faces the cytosol. The cytosolic ATPase TRC40, Get3 in yeast, binds newly synthesized TA proteins and targets them to the ER where they are inserted via a membrane receptor formed by WRB and CAML, functionally equivalent to yeast Get1 and Get2. This heteromultimeric membrane protein complex works as a docking site for TRC40 and "insertase" for TA proteins. Although several molecular aspects of the pathway have been elucidated in recent years, it is poorly understood how the pathway is integrated into the cellular proteostasis network in vivo. In order to gain insight into this question, the fate of several TA proteins (Sec61 $\beta$, syntaxins 5, 6 and 8 and emerin) was evaluated in a heart-specific, inducible knockout of WRB mouse model. Our analysis showed that endogenous syntaxin 5 and emerin are reduced upon WRB depletion. On the one hand, we observed that syntaxin 5 is degraded by autophagy upon disruption of the TRC40 pathway, constituting a novel mechanism for the clearance of TA proteins. On the other hand, our results confirm that emerin, a clinically relevant TA protein of the nuclear envelope, depends on the TRC40 pathway for its insertion into the ER and targeting to its final destination. Our results suggest the TRC40 pathway does not only contribute to insertion fidelity of its clients but also to their overall stability. 


\section{Authors contributions to the listed publications}

Pfaff, J.*, Rivera Monroy*, J., Jamieson, C., Rajanala, K., Vilardi, F., Schwappach, B. and Kehlenbach, R. H. (2015). Emery-Dreifuss muscular dystrophy mutations impair TRC40-mediated targeting of emerin to the inner nuclear membrane. J Cell Sci.

*These authors contributed equally to this work

\begin{tabular}{|c|c|c|}
\hline Figure & Method & $\begin{array}{l}\text { Experimental } \\
\text { contribution }\end{array}$ \\
\hline Fig. 1 & $\begin{array}{l}\text { Cloning of pQE80-MBP-TRC40/HZZ-emerin- } \\
\text { opsin. }\end{array}$ & $\begin{array}{l}\text { Ralph } \\
\text { Kehlenbach }\end{array}$ \\
\hline Fig. 1 & $\begin{array}{l}\text { Purification of MBP-TRC40/HZZ-emerin-opsin } \\
\text { complex. }\end{array}$ & Jhon Rivera \\
\hline Fig. 1 & $\begin{array}{l}\text { In-vitro insertion assays of emerin in ER } \\
\text { membranes. }\end{array}$ & Jhon Rivera \\
\hline Fig. 2 & Cloning of pGEM3Z-emerin-op. & Fabio Vilardi \\
\hline Fig. 2 & $\begin{array}{l}\text { In vitro translation and TRC40-dependent } \\
\text { membrane insertion assay of emerin. }\end{array}$ & Fabio Vilardi \\
\hline Fig. 3 & $\begin{array}{l}\text { Purification of MBP-TRC40/HZZ-emerin-opsin } \\
\text { complex, MBP-WRBcc, GST-CAML-N. }\end{array}$ & Jhon Rivera \\
\hline Fig. 3 & $\begin{array}{l}\text { Insertion of emerin into membranes of semi- } \\
\text { permeabilized cells. }\end{array}$ & Janine Pfaff \\
\hline Fig. 4 & $\begin{array}{l}\text { In situ PLA detection of TRC40-emerin } \\
\text { interactions. }\end{array}$ & Cara Jamieson \\
\hline Fig. 5 & $\begin{array}{l}\text { Optimization TRC40 siRNA-mediated depletion } \\
\text { in HeLa Cells. }\end{array}$ & $\begin{array}{l}\text { Jhon Rivera/ } \\
\text { Janine Pfaff }\end{array}$ \\
\hline Fig. 5 & $\begin{array}{l}\text { Immunofluorescence of emerin, LBR and } \\
\text { STT3B in siTRC40 HeLa cells. }\end{array}$ & $\begin{array}{l}\text { Cara Jamieson/ } \\
\text { Jhon Rivera }\end{array}$ \\
\hline
\end{tabular}




\begin{tabular}{|l|l|c|}
\hline Fig. 6 & $\begin{array}{l}\text { Cloning and protein purification of MBP- } \\
\text { TRC40/HZZ-emerin-opsin disease variants. }\end{array}$ & Jhon Rivera \\
\hline Fig. 6 & $\begin{array}{l}\text { In-vitro insertion assays in ER membranes of } \\
\text { emerin disease variants. }\end{array}$ & Jhon Rivera \\
\hline Fig. 7 & $\begin{array}{l}\text { Subcellular localization by IF of emerin disease } \\
\text { variants in differentially permeabilized HeLa. }\end{array}$ & Jhon Rivera \\
\hline Fig. 8 & $\begin{array}{l}\text { Cloning and protein purification of EGFP2-NLS- } \\
\text { FKBP12, WRB-FRB-HA and mCherry-FRB- } \\
\text { emerin disease variants. }\end{array}$ & $\begin{array}{c}\text { Janine Pfaff/ } \\
\text { Kalpana Rajanala }\end{array}$ \\
\hline Fig. 8 & Rapamycin assay. & $\begin{array}{c}\text { Janine Pfaff/ } \\
\text { Kalpana Rajanala }\end{array}$ \\
\hline
\end{tabular}

Rivera-Monroy, J. ${ }_{2}^{*}$ Musiol, L.*, Unthan-Fechner, K., Farkas, Á., Clancy, A., Coy-Vergara, J., Weill, U., Gockel, S., Lin, S.-Y., Corey, D. P., et al. (2016). Mice lacking WRB reveal differential biogenesis requirements of tail-anchored proteins in vivo. Sci Rep 6, 39464

*These authors contributed equally to this work

\begin{tabular}{|l|l|c|}
\hline Figure & \multicolumn{1}{|c|}{ Method } & $\begin{array}{c}\text { Experimental } \\
\text { contribution }\end{array}$ \\
\hline $\begin{array}{l}\text { All } \\
\text { Figs }\end{array}$ & $\begin{array}{l}\text { Breeding mouse colonies, genotyping and } \\
\text { knockout induction. }\end{array}$ & $\begin{array}{c}\text { Kirsten Unthan- } \\
\text { Fechner }\end{array}$ \\
\hline $\begin{array}{l}\text { All } \\
\text { Figs }\end{array}$ & $\begin{array}{l}\text { Antibody optimization for IF and WB of TRC40- } \\
\text { pathway components, Tail-anchored proteins } \\
\text { and autophagy-related proteins. }\end{array}$ & $\begin{array}{c}\text { Jhon Rivera } \\
\text { Sarah Gockel }\end{array}$ \\
\hline Fig. 1 & $\begin{array}{l}\text { mRNA analysis of TRC40-pathway components } \\
\text { in WRB KO cardiomyocytes. }\end{array}$ & $\begin{array}{c}\text { Jhon Rivera/ } \\
\text { Fabio Vilardi }\end{array}$ \\
\hline Fig. 1 & $\begin{array}{l}\text { Western blot analysis of TRC40-pathway } \\
\text { components in KO cardiomyocytes. }\end{array}$ & Jhon Rivera \\
\hline Fig. 1 & $\begin{array}{l}\text { Western blot analysis of Tail-anchored proteins } \\
\text { in WRB KO cardiomyocytes. }\end{array}$ & Jhon Rivera \\
\hline Fig. 2 & $\begin{array}{l}\text { mRNA analysis of TRC40-pathway components } \\
\text { in WRB-depleted hepatocytes. }\end{array}$ & Lena Musiol \\
\hline
\end{tabular}




\begin{tabular}{|c|c|c|}
\hline Fig. 2 & $\begin{array}{l}\text { Western blot analysis of TRC40-pathway } \\
\text { components in WRB-depleted hepatocytes. }\end{array}$ & Lena Musiol \\
\hline Fig. 2 & $\begin{array}{l}\text { Western blot analysis of Tail-anchored proteins } \\
\text { in WRB KO hepatocytes. }\end{array}$ & Lena Musiol \\
\hline Fig. 3 & $\begin{array}{l}\text { Immunofluorescence of Tail-anchored proteins } \\
\text { in KO cardiomyocytes. }\end{array}$ & Jhon Rivera \\
\hline Fig. 3 & $\begin{array}{l}\text { Immunofluorescence of Tail-anchored proteins } \\
\text { in KO hepatocytes. }\end{array}$ & Lena Musiol \\
\hline Fig. 3 & Live-cell microscopy in yeast. & $\begin{array}{l}\text { Akos Farkas/ } \\
\text { Anne Clancy }\end{array}$ \\
\hline Fig. 4 & $\begin{array}{l}\text { Cloning of pGEM3Z-Stx5-op, pGEM3Z-Stx8-op, } \\
\text { TET-induced GFP Stx5-Stx8 quimeras, pQE80- } \\
\text { MBPtevStx5cyt and pQE80-MBPtevStx8cyt. }\end{array}$ & $\begin{array}{l}\text { Fabio Vilardi/ } \\
\text { Jhon Rivera }\end{array}$ \\
\hline Fig. 4 & $\begin{array}{l}\text { In vitro translation and TRC40-dependent } \\
\text { membrane insertion assay of Stx5 and Stx8. }\end{array}$ & Fabio Vilardi \\
\hline Fig. 4 & $\begin{array}{l}\text { Levels of LC3B-II/LC3B-I in TET-induced Stx5- } \\
\text { Stx8 quimeras by western blot. }\end{array}$ & Fabio Vilardi \\
\hline Fig. 4 & $\begin{array}{l}\text { Aggregation assay and circular dichroism } \\
\text { spectroscopy of MBPtevStx5cyt and } \\
\text { MBPtevStx8cyt. }\end{array}$ & Fabio Vilardi \\
\hline Fig. 5 & $\begin{array}{l}\text { Co-transfection of FAM134B siRNA on WRB or } \\
\text { TRC40 knockdown cells. }\end{array}$ & Jhon Rivera \\
\hline Fig. 5 & $\begin{array}{l}\text { Co-transfection of p62 siRNA on WRB or } \\
\text { TRC40 knockdown cells. }\end{array}$ & Jhon Rivera \\
\hline Fig. 5 & $\begin{array}{l}\text { Western blot analysis of Stx } 5 \text { and Stx8 in } \\
\text { silenced cells. }\end{array}$ & Jhon Rivera \\
\hline $\begin{array}{l}\text { Supp. } \\
\text { Fig. } 1\end{array}$ & $\begin{array}{l}\text { mRNA levels of Tail-anchored proteins in } \\
\text { isolated cardiomyoctyes and hepatocytes by } \\
\text { qPCR. }\end{array}$ & $\begin{array}{l}\text { Lena Musiol/ } \\
\text { Fabio Vilardi/ } \\
\text { Jhon Rivera }\end{array}$ \\
\hline $\begin{array}{l}\text { Supp. } \\
\text { Fig. } 2\end{array}$ & $\begin{array}{l}\text { Western blot analysis of TRC40-pathway } \\
\text { components in cardiomyocytes from MerCreMer } \\
\text { control line. }\end{array}$ & Jhon Rivera \\
\hline $\begin{array}{l}\text { Supp. } \\
\text { Fig. } 2\end{array}$ & $\begin{array}{l}\text { Western blot analysis of Tail-anchored proteins } \\
\text { in cardiomyocytes from MerCreMer control line. }\end{array}$ & Jhon Rivera \\
\hline
\end{tabular}




\begin{tabular}{|c|c|c|}
\hline $\begin{array}{l}\text { Supp. } \\
\text { Fig. } 2\end{array}$ & $\begin{array}{l}\text { Immunofluorescence of Tail-anchored proteins } \\
\text { in MerCreMer control cardiomyocytes. }\end{array}$ & Jhon Rivera \\
\hline $\begin{array}{l}\text { Supp. } \\
\text { Fig. } 3\end{array}$ & $\begin{array}{l}\text { Colocalization of SNARE proteins with the Golgi } \\
\text { marker P115 in KO cardiomyocytes. }\end{array}$ & Jhon Rivera \\
\hline $\begin{array}{l}\text { Supp. } \\
\text { Fig. } 4\end{array}$ & $\begin{array}{l}\text { Histochemistry and Blood value measurement in } \\
\text { KO hepatocytes. }\end{array}$ & $\begin{array}{l}\text { Philipp Ströbel/ } \\
\text { Lena Musiol/ } \\
\text { Kirsten Unthan- } \\
\text { Fechner }\end{array}$ \\
\hline $\begin{array}{l}\text { Supp. } \\
\text { Fig. } 4\end{array}$ & $\begin{array}{l}\text { Protein analysis of TRC40-pathway } \\
\text { components, Stx5 and Stx8 in WRB-depleted } \\
\text { hepatocytes (Data contained partially in Fig. 2). }\end{array}$ & Lena Musiol \\
\hline $\begin{array}{l}\text { Supp. } \\
\text { Fig. } 5\end{array}$ & $\begin{array}{l}\text { Live-cell microscopy of a library genomically } \mathrm{N}- \\
\text { terminally GFP-Tagged in S. cerevisiae } \\
\text { get1/get } 2 \text {, get } 3 \text { and get1/get2/get } 3 \text {, knockout } \\
\text { strains. }\end{array}$ & $\begin{array}{l}\text { Akos Farkas/ } \\
\text { Anne Clancy/ } \\
\text { Uri Weill/ } \\
\text { Maya Schuldiner }\end{array}$ \\
\hline $\begin{array}{l}\text { Supp. } \\
\text { Fig. } 6\end{array}$ & In-vitro Aggregation assay. & Fabio Vilardi \\
\hline $\begin{array}{l}\text { Supp. } \\
\text { Fig. } 6\end{array}$ & $\begin{array}{l}\text { Lysosomal inhibition in siWRB and siTRC40 } \\
\text { HeLa and Stx5 and Stx8 western blot. }\end{array}$ & Fabio Vilardi \\
\hline
\end{tabular}




\section{Abbreviations}

Integral Membrane Protein IMP

Transmembrane Domain TMD

Tail-Anchored protein

TA protein

Endoplasmic Reticulum ER

Signal Recognition Particle SRP

Transmembrane Recognition

Complex subunit of $40 \mathrm{kDa} \quad$ TRC40

Guided Entry of Tail-anchored proteins 3 Get

Arsenical pump-driving ATPase ArsA

Tryptophan-rich basic protein WRB

congenital heart disease protein $5 \quad$ CHD5

Small glutamine-rich tetratricopeptide

repeat-containing protein alpha SGTA

Short Secretory Protein Precursor SSPP

Short Secretory Protein SSP

Glycosylphosphatidylinositols protein GPI protein

Guanine-nucleotide Exchange Factor GEF

G Protein-Couple Receptor GPCR

Mitogen-Activated Protein Kinase MAPK

Apoptosis Inducing Factor AIF

SRP-independent targeting pathway SND pathway

Ring Finger Protein $126 \quad$ RNF126

Cysteine-String Protein CSP

Mislocalized Protein MLP

Ubiquitin-Like domain Ubl domain

Emerin EMD

Emery-Dreifuss Muscular Dystrophy EDMD

Syntaxin $5 \quad$ Stx5

Syntaxin $6 \quad$ Stx6

$\begin{array}{ll}\text { Syntaxin } 8 & \text { Stx8 }\end{array}$ 


\section{Introduction}

\section{Biogenesis of integral membrane proteins of the secretory pathway}

All biological membranes contain integral membrane proteins (IMP) that are structurally diverse and collectively constitute $20-30 \%$ of the proteome of every organism. This subgroup of proteins perform an enormous range of cellular activities including communication across the membrane, transport of substances, membrane morphogenesis, among many others (Hegde and Keenan, 2011; Wallin and Heijne, 1998).

The common feature of IMPs is the presence of at least one hydrophobic transmembrane domain (TMD). TMDs allow protein anchoring thanks to its thermodynamic compatibility with the environment of the lipid bilayer (Fig. 1)

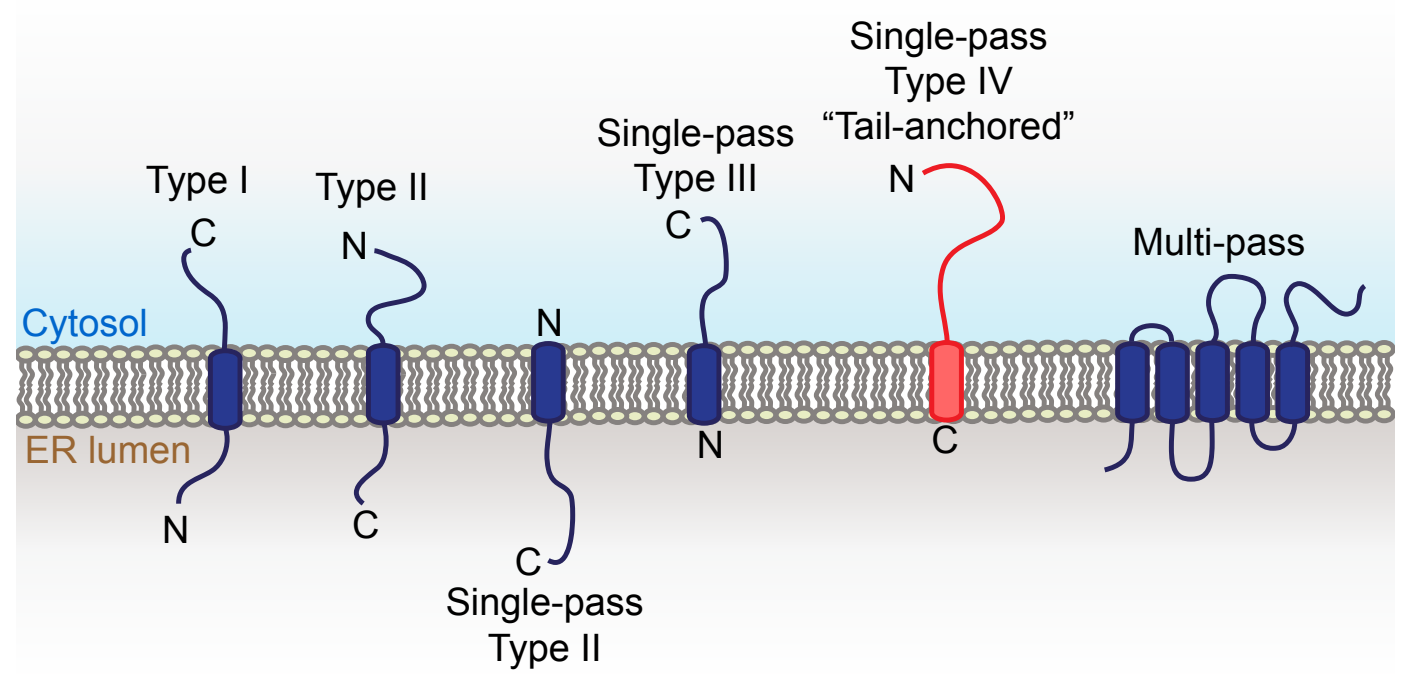

Figure 1. Classification of Integral membrane protein topology.

In contrast to soluble proteins that are synthetized in the hydrophilic cytosol, the exposure of hydrophobic regions presents a problem during 
IMP biogenesis. Several pathways for the biogenesis of membrane proteins have evolved to protect the precursor protein from aggregation, allowing at the same time the correct topological orientation of the protein into the membrane.

IMPs are targeted to the membranes of mitochondria, peroxisomes, chloroplasts and the endoplasmic reticulum (ER) (Chacinska et al., 2009; Garg and Gould, 2016; Giannopoulou et al., 2016; Hegde and Keenan, 2011). From the ER IMPs can reach all other cellular membranes connected to the secretory pathways, and also to the inner and outer nuclear membranes.

\section{Pathways for the insertion of ER membrane proteins}

Considering the central role of the ER membrane in protein biogenesis as the place of insertion of IMPs of secretory pathway compartments and the nuclear membrane, the pathways involved in membrane protein insertion at the ER have being extensively studied in the last 4 decades.

These pathways can be classified in co-translational and post-translational pathways (Fig. 2). In the co-translational pathway, the synthesis of the protein occurs in a nearly complete way on the ER membrane, synthesis and insertion occurring as a coupled process. In contrast, the membrane protein insertion via a post-translational pathway occurs after the protein is synthetized and released into the cytosol. To date, two different types of ER-targeting sequences have been characterized in eukaryotes: the cleavable $\mathrm{N}$-terminal signal sequence and the TMDs that act at the same time as membrane anchors (Ast et al., 2013).

The first and most studied co-translational pathway identified is the Signal Recognition Particle (SRP) pathway (Blobel and Dobberstein, 1975a; 
Blobel and Dobberstein, 1975b), a pathway that relies on a complex of the same name and that is conserved in all three domains of life (Pool, 2005). The SRP pathway is involved in ER membrane insertion of single- and multi-spanning proteins and also, in the translocation of soluble polypeptides from the cytosol to the ER lumen.

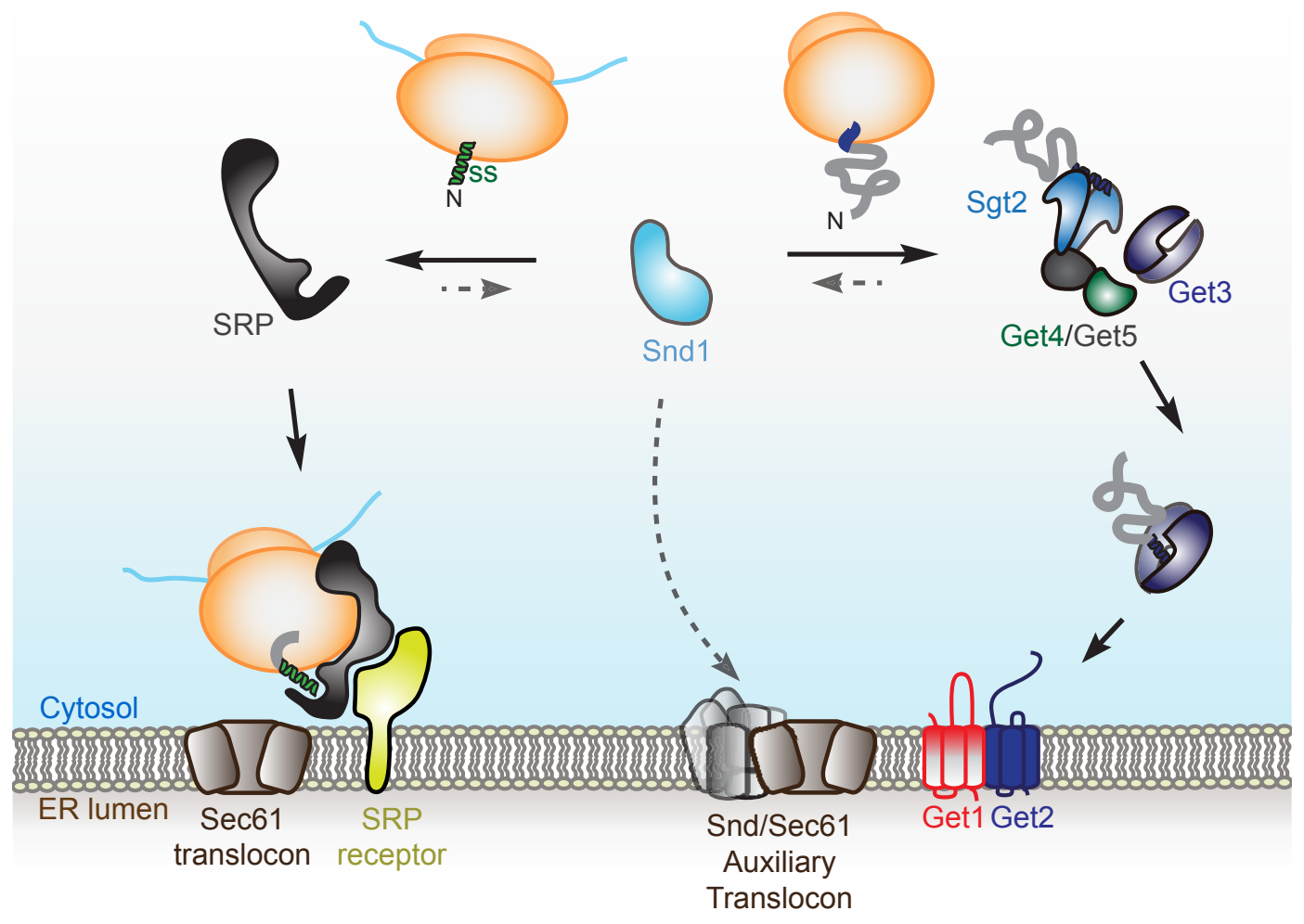

Figure 2. ER targeting pathways: Co-translational (SRP), posttranslational GET pathway and the recently discovered SND targeting components. See text below for the pertinent references.

SRP recognizes a hydrophobic $\mathrm{N}$-terminal signal sequence on the nascent polypeptide emerging from the exit tunnel of a translating ribosome. Once bound, SRP is also able to pause the translation until the SRP-ribosome macrocomplex is recruited to the Sec61 translocon at the ER membrane via the SRP receptor, where translation is resumed (Grudnik et al., 2009).

Although many ER IMPs are inserted co-translationally, several studies have shown that a subset of membrane proteins are inserted in a posttranslational manner (Kutay et al., 1993). This subset of proteins, known as single-pass type IV proteins (Fig. 1) or Tail-Anchored (TA) proteins, are 
characterized by the absence of an $\mathrm{N}$-terminal signal peptide and a single TMD towards the C-terminus (Kutay et al., 1993).

TA proteins are present in all three domains of life; in eukaryotes they represent 3 to $5 \%$ of all IMPs and are located in all cellular membranes. TA proteins exert functions related to membrane biogenesis, apoptosis (e.g., Bcl-2, Bcl- $X_{L}, B a x$ ), intracellular trafficking (e.g., most of the SNARE proteins involved in vesicular fusion are TA proteins), protein translocation (e.g. Sec61 $\beta$ and Sec61y), transcription regulation (emerin), protein maturation, degradation, organelle structure, and lipid homeostasis, among others (Hegde and Keenan, 2011; Jonikas et al., 2009; Kalbfleisch et al., 2007; Schuldiner et al., 2008; Shao and Hegde, 2011; Wereszczynski and McCammon, 2012).

The TMD of TA proteins provides a targeting signal for the delivery of the newly synthesized TA protein into the target membrane (i.e. ER, peroxisomes, outer mitochondrial membrane, and the plastid outer envelope) and acts as an anchor that retains the TA protein in the membrane once the integration has taken place (Borgese and Fasana, 2011; Rabu et al., 2009). Recent work has suggested that more than one post-translational pathway operates in ER targeting of TA proteins. In vitro studies have shown the existence of (i) an unassisted insertion pathway by which proteins with moderate hydrophobic TMDs spontaneously integrate into the membrane (Colombo et al., 2009) and (ii) assisted chaperone-mediated, energy-requiring pathways, by which most ERtargeted TA proteins reach their destination (Borgese and Fasana, 2011). So far four post-translational assisted pathways have been described: one mediated by SRP itself (Abell et al., 2004), one by the Hsc70/Hsp40 chaperone complex (Abell et al., 2007), one mediated by the recently described SND components (Aviram et al., 2016), and one by the Transmembrane Recognition Complex subunit of $40 \mathrm{kDa}$ (TRC40) in mammals or by Guided Entry of Tail-anchored proteins 3 (Get3) in yeast 
(Favaloro et al., 2008; Schuldiner et al., 2008; Stefanovic and Hegde, 2007). Among them, The TRC40/Get3 is currently thought to be the preferential insertion pathway for most of the TA proteins.

\section{The TRC40/Get3 pathway}

Mammalian TRC40 (also known as Asna1) and its yeast counterpart Get3 (Arr4) are cytosolic P-loop ATPase homodimers that bind the TMD of TA proteins and deliver them to the ER membrane (Favaloro et al., 2008; Schuldiner et al., 2008; Stefanovic and Hegde, 2007). The GET3/ASNA1 genes are homologous to the arsenical pump-driving ATPase (ArsA), thought to function as a regulatory component of the bacterial arsenic export pump (Boskovic et al., 1996; Karkaria et al., 1990; Kaur and Rosen, 1992; Rosen et al., 1988).

Genetic and biochemical approaches have shown that several proteins interact with Get3 during the biogenesis of TA proteins as part of the yeast GET pathway (Auld et al., 2006; Jonikas et al., 2009; Schuldiner et al., 2008), most of them are conserved from yeast to mammals (Table 1). Get1 and Get2 are membrane proteins that associate in a heterooligomeric complex and recruit Get3 to the ER membrane (Jonikas et al., 2009; Schuldiner et al., 2008), whereas Get4 and Get5 interact with the Sgt2 protein in the capture of newly synthesized TA proteins at the ribosome and their delivery to Get3 (Battle et al., 2010; Chang et al., 2010; Jonikas et al., 2009; Simpson et al., 2010).

Structural studies have shown that the interaction of Sgt2 and the Get4Get5 complex is mediated by the ubiquitin-like domain of Get5 (Chartron et al., 2012; Wang et al., 2010). The C-terminal region of Sgt2 is thought to bind to the TMD of the nascent TA protein right after it emerges from the ribosome, thus bringing the TA proteins into the vicinity of the Get4Get5 complex (Wang et al., 2010). The Sgt2-Get4-Get5 complex recruits ATP-bound Get3 via the interaction between Get4 and Get3, inhibiting the 
ATPase activity of Get3. This facilitates the transfer of the TA protein from Sgt2 to Get3, which also binds the TA protein TMD (Chang et al., 2012; Chartron et al., 2010). Once Get3 is loaded with the TA protein, the ATPase activity of Get3 is enhanced (Rome et al., 2013), leading to the formation of an ADP-bound Get3-TA protein complex, which is competent for ER membrane targeting.

This targeting-competent complex is initially recruited to the membrane through an interaction of Get3 with a negatively charged region in the $\mathrm{N}$ terminal domain of Get2 (Mariappan et al., 2011; Stefer et al., 2011). Subsequently, the cytosolic coiled-coil domain of Get1 interacts with Get3 to drive TA protein release concomitantly with ADP release.

The final membrane integration step has been a matter of extensive discussion since the receptor was described. Using a biochemical reconstitution approach, it was recently demonstrated that the Get1-Get2 receptor plays a direct role as an insertase during the insertion of TA proteins into the ER membrane (Wang et al., 2014). According to this report, the TMDs of Get1 and Get2 interact with the TMD of the TA protein close to the cytosolic surface of the ER bilayer and collaborate with the cytosolic domains of the Get1-Get2 receptor to release the TA substrate from the open conformation of Get3, thus allowing integration of the TA protein TMD into the membrane.

Like in yeast, the capture of TA proteins in mammals by ATP-bound TRC40 is facilitated by an upstream pretargeting complex (Fig. 3) thought to associate with the ribosome. This complex is formed by BAG6 (Bat3), TRC35, and UBL4A; where the latter two proteins are homologs of Get4 and Get5, respectively (Leznicki et al., 2010; Mariappan et al., 2010). Although mammalian BAG6 and yeast Sgt2 are not related, it is noteworthy that BAG6 interacts with SGTA, the mammalian homologue of Sgt2 (Winnefeld et al., 2006). 
Table 1. Components of the TRC40/Get3 pathways

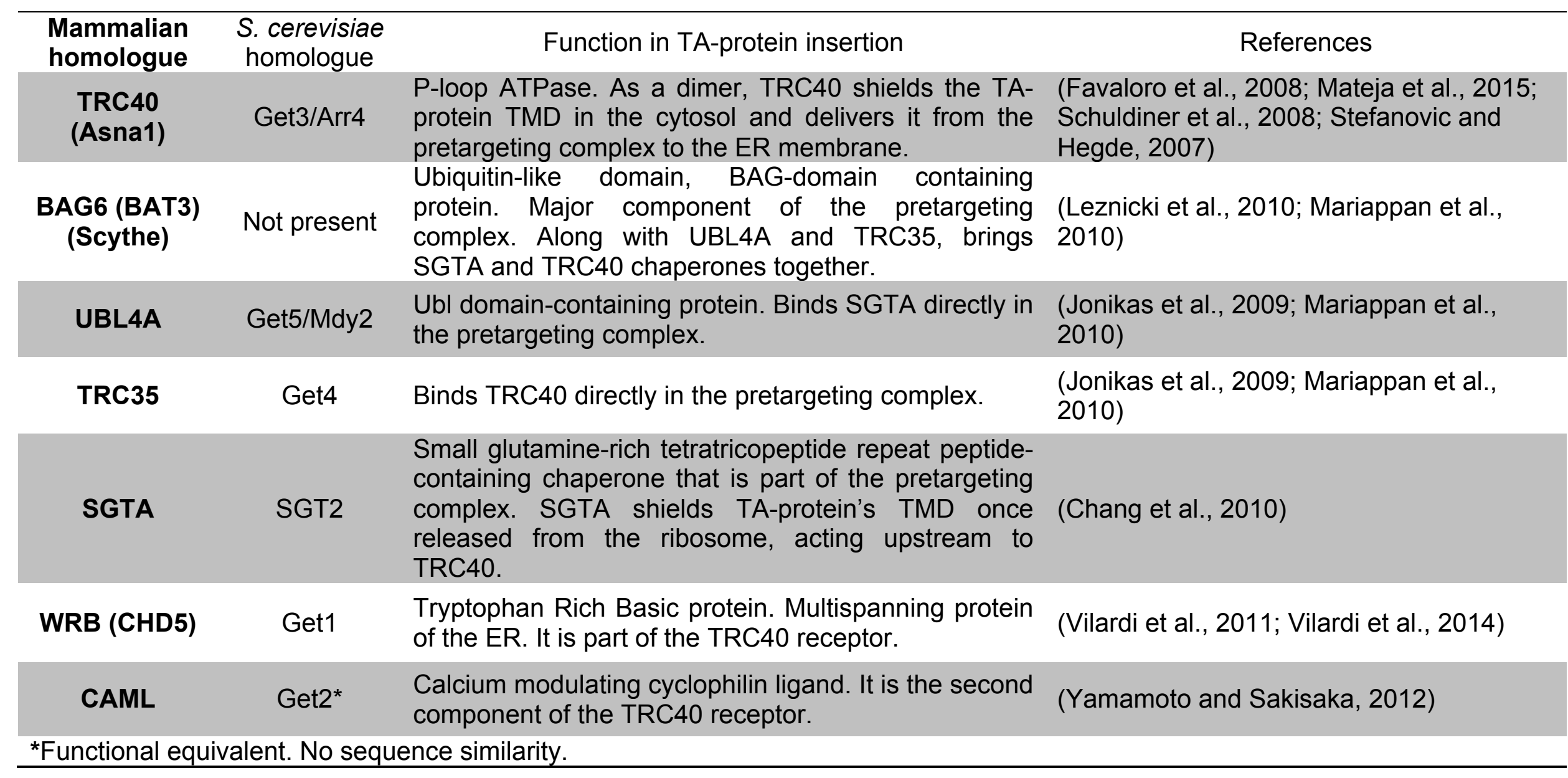




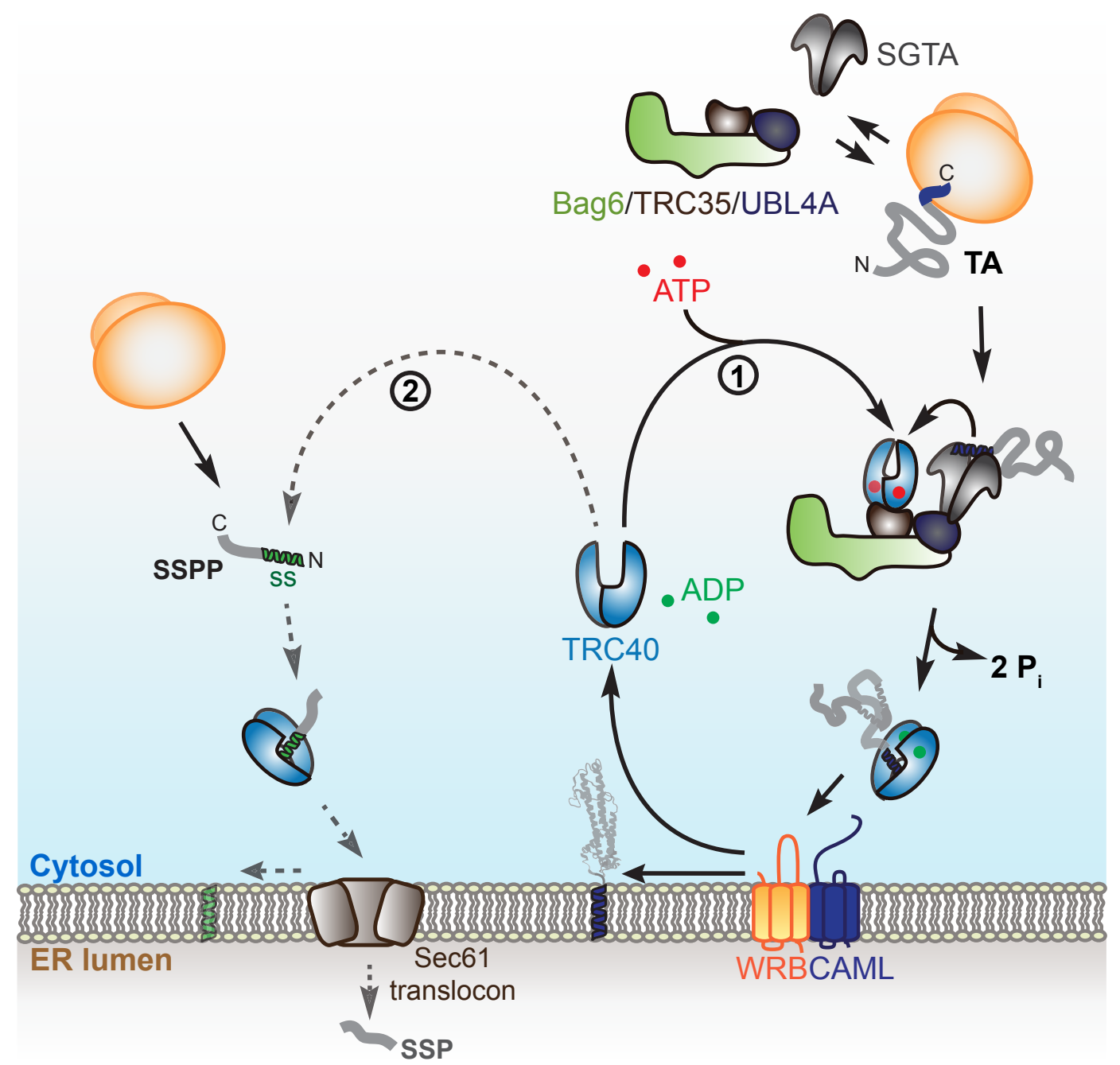

Figure 3. Roles of the mammalian TRC40 ATPase. TRC40 can target substrates of different nature to the ER membrane: 1. Post-translational insertion of TA proteins into the membrane. 2. Post-translational translocation of short secretory proteins SSP. SS: Signal Sequence, SSPP: Short secretory protein precursor. As indicated by the dashed line current insight into the mechanism of SSPP targeting via TRC40 is limited.

It was demonstrated that the ER proteins WRB (homologue of Get1) and CAML (functional equivalent of Get2), form the receptor complex for TA protein insertion in mammals (Vilardi et al., 2011; Vilardi et al., 2014; Yamamoto and Sakisaka, 2012). Taking into account recent studies that suggest that Get1/Get2 receptor work as an insertase (Wang et al., 2014) it seems plausible to hypothesize that WRB and CAML fulfill the same function in the mammalian ER (Fig. 4). 


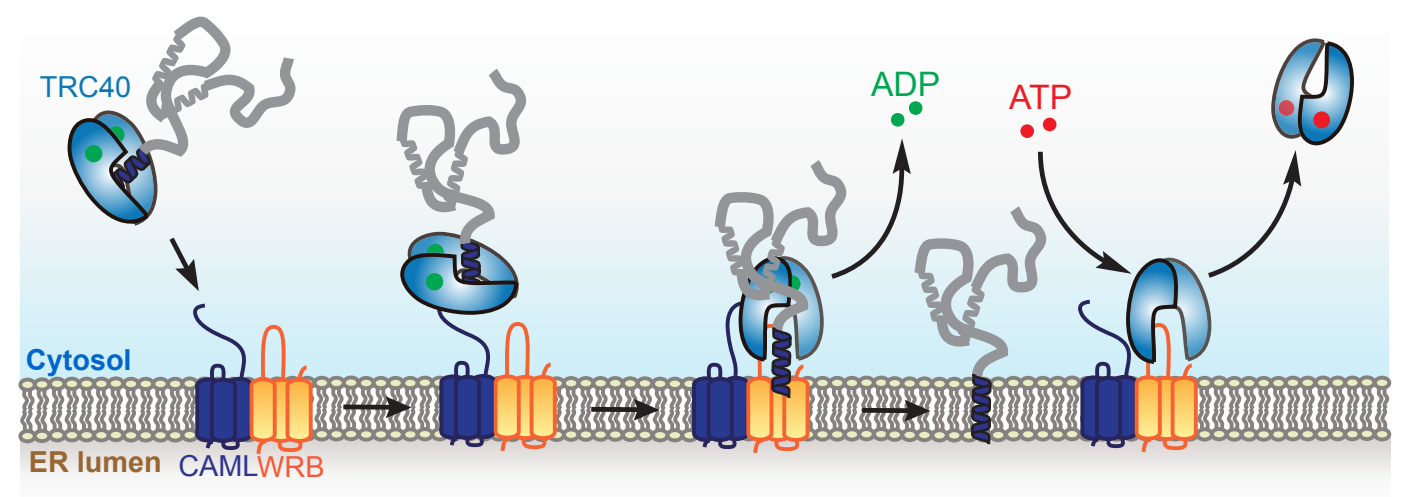

Figure 4. Proposed mechanism of the WRB/CAML receptor as an insertase.

\section{Alternative roles of the TRC40/Get3 ATPases}

Apart from the well-known ATP-dependent targeting of TA proteins to the ER membrane, TRC40 is also involved in the translocation of short secretory peptides across the ER bilayer (Johnson et al., 2012) and potentially in GPI-anchored proteins biogenesis in an SRP-independent manner (Ast et al., 2013).

In-vitro studies carried out by Johnson et al. identified two human short secretory protein precursors, apelin and statherin, as bona fide substrates of TRC40 (Johnson et al., 2012). Both proteins interact with TRC40 and use this protein for their delivery and post-translational translocation across the ER membrane in a mechanism that appeared to be Sec61 translocon-dependent. Moreover, the cytosolic coiled-coil fragment of WRB, inhibits such translocation in vitro, suggesting the TRC40 receptor might be also involved during substrate translocation.

Combining hydrophathy-based analysis and high-throughput microscopy Ast et al. (2013) evaluated the prevalence SRP-independent pathway(s) in the translocation of secretory proteins in yeast. This screen revealed that a subset of GPI-anchored proteins containing a hydrophobic C-terminal targeting element required for their eventual modification with a glycanlipid anchor, are targeted by the GET pathway. The GET dependence was 
direct as Get3 interacted with the GPI-anchored HA-Gas1; furthermore, this dependence correlated with a high hydrophobicity of the C-terminus in the GPI-anchored proteins. Such a correlation has also been observed in the targeting of TA proteins in vitro (Favaloro et al., 2010; Jonikas et al., 2009; Leznicki et al., 2010; Schuldiner et al., 2008; Stefanovic and Hegde, 2007).

Recently, our lab has described novel functions of Get3 as a holdase chaperone upon stress conditions, such as energy depletion or oxidative stress (Powis et al., 2013b; Voth et al., 2014). Powis et al. (2013) observed that Get3 rapidly relocalizes to foci following glucose starvation. These foci resulted to be protein-rich, membrane and ribosome-free deposition sites where aggregated TA proteins (i.e. the Sed5 SNARE protein) and other chaperones and disaggregases (i.e. Hsp42, Hsp104, Sis1 and Ssa2) were also found together with Get3. The localization of Get3 in foci was not observed in the knockout of the cytosolic components Get4 and Get5; in contrast, in a get1/get2 strain (receptor KO) foci were observed even without exposing the cells to stress.

In-vitro studies clearly showed that Get3 acts as a chaperone holdase when ATP is not available. Moreover, addition of adenosine nucleotides strongly inhibited this function (Voth et al., 2014). The D57E Get3 mutant, a mutant protein with an impaired ATP-hydrolysis activity but preserved TA protein binding, accumulates in foci irrespective of glucose availability which is consistent with the observed change of localization of the protein when cells are glucose-starved (Powis et al., 2013a).

Voth et al. (2014) demonstrated that Get3 turns into an ATP-independent redox-regulated chaperone in yeast. Get3 is a zinc-binding protein that presents four conserved cysteines arranged in a $\mathrm{C}-\mathrm{X}-\mathrm{C}-\mathrm{X}_{43}-\mathrm{C}-\mathrm{XX}-\mathrm{C}$ motif (corresponding to the region $\mathrm{C} 240$ to $\mathrm{C} 288$ ). This arrangement is very 
similar to the oxidation sensitive zinc-binding motif present in the unrelated protein Hsp33, a bacterial redox-regulated ATP-independent chaperone.

The incubation of Get3 with $\mathrm{H}_{2} \mathrm{O}_{2}$ and $\mathrm{Cu}^{2+}$, that induces hydroxyl radical production, promotes the activation of Get3 as a chaperone holdase. Under these conditions, Get3 undergoes massive conformational changes, triggered by disulphide bond formation and zinc release. Get3 rearrangement results in the formation of highly active tetramers and higher oligomers that can bind unfolding proteins, preventing their aggregation. Interestingly, $H / D$ exchange experiments on reduced and oxidized Get3 showed that the region that contains the hydrophobic binding site for TA proteins (aa184-201) is the less affected in terms of exposure, which in line with the function of Get3 as a redox regulate chaperone.

Synthetic genetic array (SGA) analysis in Saccharomyces cerevisiae identified genetic interactions under heat shock of GET3, GET1 and GET2 with NAS6, a chaperone involved in the assembly of the $19 S$ regulatory particle of the proteasome as well as genes encoding other proteasome assembly chaperones such as HSM3, RPN14 and UMP1 (Akahane et al., 2013).

Knockdown of TRC40 in HEK293T cells caused accumulation of ubiquitinated proteins, reduced peptidase activity of the $20 \mathrm{~S}$ proteasome (core particle) as well as increased levels of the immature 205 proteasome. Moreover, Bag6 depleted cells exhibited a defect in the core particle quite similar to what was observed in the TRC40 knockdown, suggesting that the role of TRC40/Get3 pathway in proteasome assembly in conserved from yeast to humans (Akahane et al., 2013).

An extra role of Get3 in signalling mediated by $G$ protein was also proposed in yeast (Lee and Dohlman, 2008). Like G protein-couple 
receptors (GPRCs), Get3 binds directly the G protein Gpa1 accelerating its guanine-nucleotide exchange. This promotes $G$ protein-dependent cellular responses such as mitogen-activated protein kinase (MAPK) phosphorylation. Nevertheless, this role has not been evaluated in the case of TRC40.

\section{Redundancy of the insertion pathways}

Despite the number of substrates handled by the cotranslational SRP and the post-translational TRC40/Get pathways, many proteins are known to be targeted independently of both routes (Aviram and Schuldiner, 2014). These alternative pathways have been extremely difficult to investigate, particularly in vivo. A recent study provided important insights in a novel pathway, known as the SRP-independent targeting (SND) pathway (Aviram et al., 2016). Using a systematic visual screen in Saccharomyces cerevisiae, Aviram et. al (2016) described three uncharacterized proteins that are involved in the ER targeting of Gas1, a SRP-independent and partially Get3-dependent substrate (Ast et al., 2013). The proteins identified in the screening, named Snd1, Snd2 and Snd3, act as a backup targeting system when the SRP and the Get pathways are impaired.

This study suggests that the SND components might target a very diverse range of proteins, particularly substrates with central TMDs. The localization of the first TMD within the proteins seems to play a major role also in determining the dependency on the SND pathway. Although the molecular mechanisms of the SND pathway have not been address yet, it is tempting to speculate the way the SND pathway operates, based on parallels with the SRP and Get pathways: Snd1, which is thought to be a peripherical ribosomal protein (Huh et al., 2003), might be involved in capturing substrates at the ribosome to funnel them to the ER membrane through a dedicated receptor (Fig. 2). The receptor might be composed by Snd2, Snd3, which form a complex with the Sec61 translocon at the ER 
membrane, based on the result of a blue-native PAGE analysis (Aviram et al., 2016).

Nevertheless, it is not clear whether the SND pathway is also present in higher eukaryotes as the only component of the pathway with a direct homologue in mammals is Snd2 (TMEM208).

\section{Animal models of the TRC40-pathway components}

Some of the TRC40 pathway components have been associated with human health and disease. For instance, some evidence suggests that the ER receptor component WRB, might be involved in congenital heart disease, an endemic disorder occurring in $1 \%$ of all live births (Benjamin et al., 2017). WRB was initially identified in a screen for genes associated to heart disease in Down's syndrome (DS) patients in a restricted region within the chromosome 21 (Egeo et al., 1998) and for that reason WRB is also known as congenital heart disease protein 5 (CHD5). Moreover, the number of DS patients with congenital heart disease is rather high (35$60 \%$ of individuals affected) (Chaoui et al., 2005; Goodship et al., 1998). Nevertheless, the precise role of the WRB/CHD5 during heart morphogenesis and physiology is still poorly understood.

In the attempt to examine the physiological impact of the TRC40 pathway in vivo, several knockout and knockdown models of different components have been generated in different model organisms over the years (Table 2). However, the identification of TA substrates of the pathway in vivo as well as how the pathway is integrated into the cellular proteostasis network is poorly understood. 
Table 2. Animal models of TRC40-pathway components.

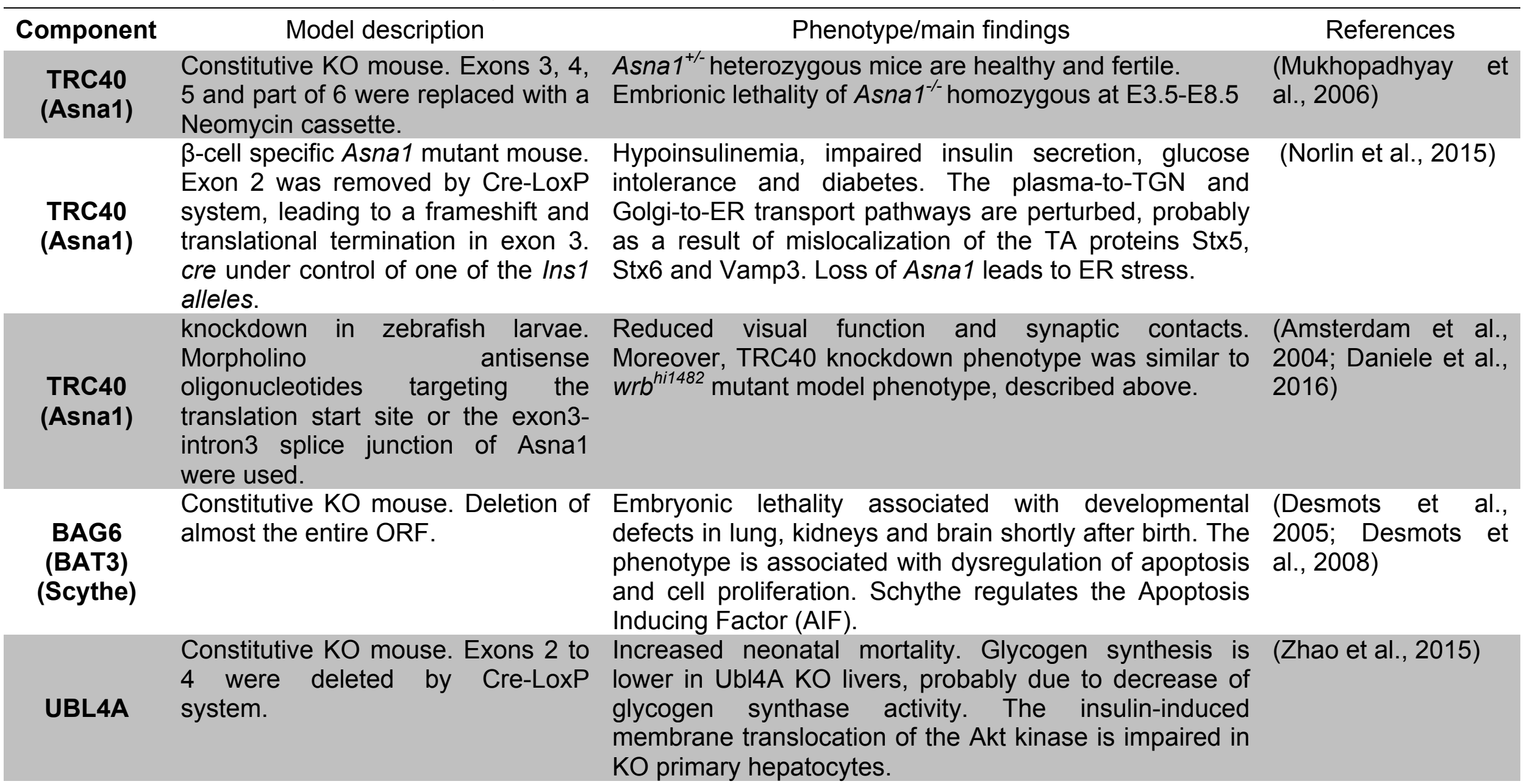




\begin{tabular}{|c|c|c|c|}
\hline SGTA & $\begin{array}{l}\text { Cre-loxP Sgta null mouse. Sgta } \\
\text { gene was floxed at exons } 4 \text { and } 5 \text {, } \\
\text { which removed the TPR1 motif and } \\
\text { part of the TPR2 motif. }\end{array}$ & $\begin{array}{l}\text { Sgta }^{+/-} \text {animals are fertile while Sgta } \\
\text { higher neonatal death. SGTA-null animals present a } \\
\text { mild phenotype of reduced body size and increase in } \\
\text { androgen-sensitive organs. }\end{array}$ & (Philp et al., 2016) \\
\hline $\begin{array}{c}\text { WRB } \\
\text { (CHD5) }\end{array}$ & $\begin{array}{l}\text { Knockdown in medaka fish (Oryzias } \\
\text { latipes) embryos. CHD5 initiation } \\
\text { translation was blocked with a } \\
\text { specific morpholino antisense } \\
\text { oligonucleotide. }\end{array}$ & $\begin{array}{l}\text { WRB mRNA and protein were found to be expressed } \\
\text { after stage } 28 \text { in developing heart (ventricle and atrium). } \\
\text { WRB depletion caused a severe cardiac disorder (i.e. } \\
\text { abnormal looping and chamber formation) and ocular } \\
\text { abnormalities (i.e. cyclopy) by an unknown mechanism. }\end{array}$ & (Murata et al., 2009) \\
\hline $\begin{array}{c}\text { WRB } \\
\text { (CHD5) }\end{array}$ & $\begin{array}{l}\text { Knockdown Xenopus laevis } \\
\text { embryos. CHD5 was targeted with a } \\
\text { specific morpholino antisense } \\
\text { oligonucleotide. }\end{array}$ & $\begin{array}{l}\text { Compromised myocardial integrity, improper deposition } \\
\text { of the basement membrane, myocydial tissue adhesion } \\
\text { defects and also abnormal cardiac looping and chamber } \\
\text { formation. CHD5 is essential for the proper function of } \\
\text { the transcription factor CASTOR (CASZ1) during cardiac } \\
\text { morphogenesis. }\end{array}$ & (Soj \\
\hline $\begin{array}{c}\text { WRB } \\
\text { (CHD5) }\end{array}$ & $\begin{array}{l}\text { Trangenic } \text { wrbhi }^{1482} \text { mutant } \\
\text { zebrafish embryos. wrb }{ }^{\text {hi1482 }} \text { mutant } \\
\text { is a null allele that presents an } \\
\text { insertion of } 65 \text { bp of retroviral DNA } \\
\text { within the first intron of the wrb } \\
\text { gene. }\end{array}$ & 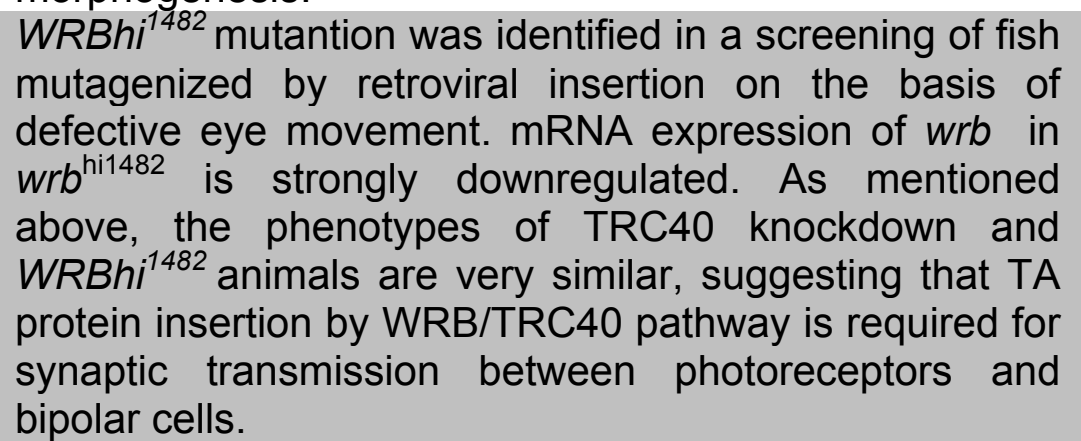 & $\begin{array}{l}\text { (Amsterdam et al., } \\
\text { 2004; Daniele et al., } \\
\text { 2016) }\end{array}$ \\
\hline
\end{tabular}


Trangenic $p w i^{\text {hi1482 }}$ mutant zebrafish embryos. pwi hi1482 is a null allele with proviral insertion at $65 \mathrm{bp}$ after the first exon of wrb gene.

\section{WRB}

(CHD5)

$\begin{array}{cl} & \text { Conditional inner hair cells } \\ & \text { knockout. Exons } 2 \text { to } 4 \text { of wrb locus } \\ \text { WRB } & \text { were removed by using Cre-LoxP } \\ \text { (CHD5) } & \text { system. cre is under control of the } \\ & \text { hair cell-specific vglut3 promoter. }\end{array}$

CAML Constitutive knockout

Conditional knockout by using Cre-

CAML LoxP system. cre is under control of the Ick promoter, active in $\mathrm{CD}^{-}$ CD8 thymocytes.

Conditional, tamoxifen-inducible

CAML knockout in inner hair cell. cre is under control of Atoh1
Deafness and blindness, with a clear phenotype on hair cells and photoreceptors. pwi ${ }^{\text {hi1482 }}$ mutation results in smaller postsynaptic responses in afferent neurons during startle auditory stimuli and impaired transmission at the photoreceptor ribbon synapse. These functional deficits were supported by a concomitant reduction of synaptic markers (Rab3 and CSP). Synaptobrevin 2 and syntaxin 3 , TA proteins involved in membrane fusion, are reduced in mutant retinas.

(Lin et al., 2016)

Hearing impairment. WRB depleted Inner hair cells display fewer ribbon-associated vesicles and impaired synaptic vesicle replenishment. Otoferlin, a TA protein involved in synaptic vesicle fusion and replenishment, is reduced in $\mathrm{KO}$ hair cells. Otofelin overexpression partially rescues the phenotype in the knockout.

CAML knockout is embryonically lethal at an early stage (between 7.5 to 12.5 days postcoitum).

(Tran et al., 2002)

CAML is essential for the development of peripherical T cell. Phosphorylation p56lck and ZAP-70 tyrosine kinases and production of IL2 is increased in CAML depleted cells. Thymocyte numbers are significantly reduced in the $\mathrm{KO}$.

Deafness as consequence of loss of cochlear hair cells.

(Bryda et al., 2012) 


\section{Quality control of TA proteins}

Due to their spontaneous propensity to aggregate, the biogenesis of TA proteins involves the protection of the TMD from the aqueous media by targeting factors such as TRC40/Get3. However, these targeting factors may fail to protect the precursor TA protein implying that quality control pathways for the degradation of mislocalized membrane proteins (MLPs) must also be integrated to the targeting process.

Recent in-vitro studies have shown that the pretargeting component Bag6 links the pathways of targeting and ubiquitination as it has not only a role during delivery of TA proteins to the ER but also a central function during clearing of MLPs, including TA proteins that failed to engage the TRC40 pathway (Hessa et al., 2011). Under normal conditions, TA proteins clients of Bag6 are transferred from SGTA to TRC40, whereas the remainder are efficiently ubiquitinated and degraded by the proteasome. Indeed, it is thought that Bag6 is able to distinguish MLPs from nascent cytosolic proteins, as MLPs present long hydrophobic stretches, while nascent cytosolic proteins contain shorter or moderately hydrophobic segments.

Based on in-vitro crosslinking, Hessa et al. (2011) identified the E3 ligase ring finger protein 126 (RNF126) and the E2 enzyme UbcH5, which are required for the ubiquitylation of MLPs, including the TA protein Sec61 $\beta$. However, the ubiquitylation of this TA substrate in the presence of TRC40 was slower compared to the ubiquitylation of the mammalian prion protein (PrP), a MLP from the class of GPI-anchored proteins. Remarkably, the depletion of TRC40 during the assay resulted in ubiquitylation of Sec61 $\beta$, suggesting that targeting to the ER membrane is favoured compared to degradation. 
Bag6 is a long protein of $\sim 1200$ amino acids with several functional domains (Shao and Hegde, 2016): the C-terminus of Bag6 mediates the transfer of the TA protein to TRC40 by forming a complex with the cofactors TRC35 and Ubl4A (Mock et al., 2015), the middle region binds the substrate (MLP) (Leznicki et al., 2013) and its N-terminus contains an ubiquitin-like domain (Ubl domain) that is responsible of the recruitment RNF126 and subsequently UbcH5 (Hessa et al., 2011).

Leznicki et. al. (2012) showed that the Bag6-dependent ubiquitylation of MLPs is completely reversible in a process that involves SGTA. Although the molecular mechanism behind this phenomenon is not well understood, it is clear that SGTA promotes the deubiquitylation by a deubiquitinase (probably UCH37), regulates the access of MLPs to the proteolytic core of the proteasome, and competes with RNF126 for Bag6-Ubl, providing a rescue pathway for potentially viable TA proteins that were prematurely ubiquitylated by Bag6 (Krysztofinska et al., 2016; Leznicki and High, 2012; Leznicki et al., 2015; Wunderley et al., 2014).

Nevertheless, it is important to highlight that Bag6 is not present in yeast, which opens the question how is the quality control of TA proteins and MLPs on this organism. Previous studies in Saccharomyces cerevisiae showed that the disruption of GET system components exacerbates the accumulation of mislocalized ER TA proteins to mitochondria (Jonikas et al., 2009; Schuldiner et al., 2008). Recently, an evolutionarily conserved system that clears mistargeted TA proteins on the outer mitochondrial membrane (OMM) was described in yeast and mammals by two groups in parallel (Chen et al., 2014; Okreglak and Walter, 2014). It was demonstrated that the AAA+ ATPase Msp1 not only interacts with the ER TA proteins Pex15 and Gos1, but also actively extracts them from OMM, limiting their mislocalization to mitochondria in yeast (Chen et al., 2014). Knockout of MSP1 resulted in growth defects and severe mitochondrial damage, particularly when combined with GET pathway knockouts. These 
growth defects were particularly severe in the double-deletion strains of

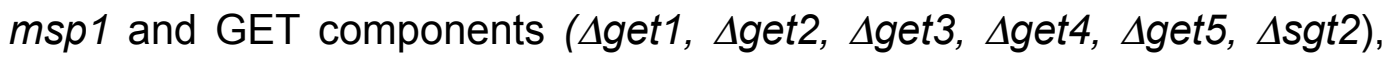
especially when they were grown on glycerol-containing medium (in which mitochondrial respiration is required) or under heat shock conditions.

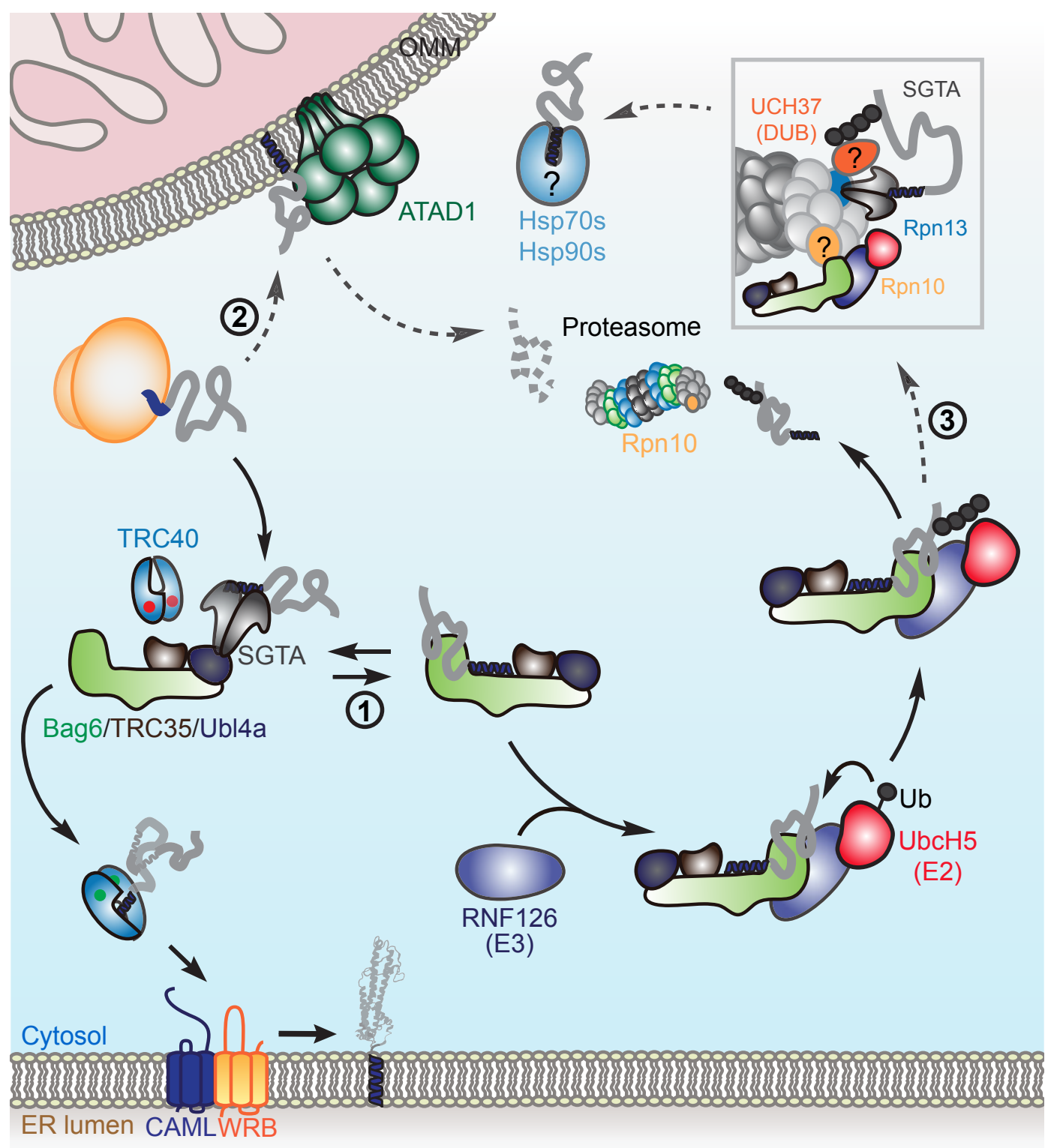

Figure 5. Clearance of mislocalized ER TA proteins from cytosol (1) and from the mitochondria (2). SGTA limits Bag6-mediated degradation probably at the proteasome (3). Ubiquitin receptors (Rpn10 and Rpn13) might recruit Bag6, SGTA and a deubiquitinase(s) to the 19S particle of the proteasome, where cycles of ubiquitylation/deubiquitylation could occur. 
The mitochondrial/respiration phenotype observed was more severe in the cases of double-deletion mutants of msp1 with $\Delta g e t 1, \Delta g e t 2$ or $\Delta g e t 3$. This phenotype was characterized by a strong reduction of mtDNA-encoded (i.e. Cox2, Cox3) and nuclear-encoded mitochondrial proteins (i.e. Atp2, Sdh1, Sdh2, Por1 and Om45), due, at least In part, to lack of functional mtDNA.

Msp1 is homolog of the mitochondrial-peroxisomal ATAD1 in mammals (Okreglak and Walter, 2014). Like Msp1, ATAD1 limits the mitochondrial mislocalization of the ER TA proteins PEX26 and GOS28, orthologs of yeast Pex15 and Gos1, respectively. Moreover, depletion of ATAD1 in an $A T A D 1^{-/}$mouse leads to decreased levels of mitochondrial proteins (i.e. TOMM20, COX1, COX4, HXK1, VDAC1) and mitochondrial fragmentation, which correlates the results obtained in yeast (Okreglak and Walter, 2014).

Nevertheless, the role of quality control pathways in the clearance of other ER-directed TA-proteins in different tissues needs to be evaluated. 


\section{Scope of the thesis}

Although the TRC40/Get pathway is considered as the main targeting system of TA proteins, a direct role in vivo has been confirmed only for a very small number of TA proteins. Moreover, considering the recent description of redundant targeting components (i.e. SNDs), it is timely to rigorously identify the TA-proteins that depend, on TRC40/Get3 at the steady-state level.

This work aims at gaining a deeper understanding of the physiological role of the TRC40 pathway during the biogenesis of candidate TA proteins in native terminally differentiated cells of the mouse. Along this line, the integration of the pathway with the cellular proteostasis network in vivo, where quality control pathways also play a major role, is considered. I focus on the mechanism behind the integration and trafficking of emerin, a TA protein that is targeted to the INM. Emerin was selected due to its unique role in muscle cells and its clinical relevance in the Emery-Dreifuss Muscular Dystrophy and because several mutations affecting emerin's TMD have been reported in patients.

As the fundamental model of the present study, I have generated and characterize here a heart-specific, tamoxifen-inducible knockout of WRB. Complementary in-vivo and in-vitro approaches are used to follow the fate of several TA proteins once the TRC40 pathway is impaired; moreover, the involvement of different degradation systems under this condition is evaluated. 


\section{Results}

Manuscript 1: Emery-Dreifuss muscular dystrophy mutations impair TRC40-mediated targeting of emerin to the inner nuclear membrane. 


\title{
Emery-Dreifuss muscular dystrophy mutations impair TRC40-mediated targeting of emerin to the inner nuclear membrane
}

\author{
Janine Pfaff ${ }^{1, *}$, Jhon Rivera Monroy ${ }^{1, *}$, Cara Jamieson ${ }^{1}$, Kalpana Rajanala ${ }^{1}$, Fabio Vilardi ${ }^{1}$, \\ Blanche Schwappach ${ }^{1,2, \pm}$ and Ralph H. Kehlenbach ${ }^{1, \pm}$
}

\begin{abstract}
Emerin is a tail-anchored protein that is found predominantly at the inner nuclear membrane (INM), where it associates with components of the nuclear lamina. Mutations in the emerin gene cause EmeryDreifuss muscular dystrophy (EDMD), an X-linked recessive disease. Here, we report that the TRC40/GET pathway for post-translational insertion of tail-anchored proteins into membranes is involved in emerin-trafficking. Using proximity ligation assays, we show that emerin interacts with TRC40 in situ. Emerin expressed in bacteria or in a cell-free lysate was inserted into microsomal membranes in an ATP- and TRC40-dependent manner. Dominant-negative fragments of the TRC40-receptor proteins WRB and CAML (also known as CAMLG) inhibited membrane insertion. A rapamycin-based dimerization assay revealed correct transport of wild-type emerin to the INM, whereas TRC40-binding, membrane integration and INMtargeting of emerin mutant proteins that occur in EDMD was disturbed. Our results suggest that the mode of membrane integration contributes to correct targeting of emerin to the INM.
\end{abstract}

KEY WORDS: CAML, TRC40, WRB, Emerin, Inner nuclear membrane, Tail-anchored protein

\section{INTRODUCTION}

The nuclear envelope, which separates the nuclear and the cytoplasmic compartments, comprises three functional domains. The outer nuclear membrane (ONM) is continuous with the endoplasmic reticulum (ER) and thus equipped with a very similar set of membrane proteins and is also studded with ribosomes. In contrast, the inner nuclear membrane (INM) contains a distinct set of membrane proteins, some of which interact with the underlying nuclear lamina and/or with chromatin. Both membranes are connected at the level of the nuclear pore complex (NPC), a large multi-protein structure that mediates transport of macromolecules between the nucleus and the cytoplasm. Hence, the ONM, the INM and the small membrane patches within the NPCs can be considered as three components of a single membrane system.

A large number of proteins that are specific for, or at least enriched at, the INM have been identified, mostly by proteomic approaches (Korfali et al., 2012; Schirmer et al., 2003). One of the best-characterized INM proteins is emerin, a member of the LEM-

${ }^{1}$ Department of Molecular Biology, Faculty of Medicine, Georg-August-University GZMB, Humboldtallee 23, Göttingen 37073, Germany. ${ }^{2}$ Max-Planck Institute for Biophysical Chemistry, Göttingen 37077, Germany.

*These authors contributed equally to this work

${ }^{\ddagger}$ Authors for correspondence (blanche.schwappach@med.uni-goettingen.de; rkehlen@gwdg.de)

Received 19 August 2015; Accepted 14 December 2015 domain family of proteins, which also contains the laminaassociated polypeptide 2 beta (LAP2 $\beta$; Foisner and Gerace, 1993; Furukawa et al., 1995) and MAN1 (also known as LEMD3) (Lin et al., 2000). The LEM-domain is a helix-loop-helix fold of $\sim 40$ amino acid residues that serves as a binding site for the chromatinassociated protein barrier to autoregulation factor (BAF, also known as BANF1). Emerin was originally identified as an X-linked gene that is mutated in patients with a certain form of Emery-Dreifuss muscular dystrophy (EDMD; Bione et al., 1994), a disease that leads to progressive skeletal muscle weakness and wasting as well as cardiomyopathies. Although a complete loss of emerin does not result in a particularly strong phenotype in knockout mice (Ozawa et al., 2006), several mutations that lead to single amino acid changes and/or frameshifts have been identified in patients affected by EDMD (see the EDMD database at http://www.dmd.nl/nmdb/ home.php?select_db=EMD). Most of these mutations lead to premature termination of translation of the emerin mRNA and to loss of protein or unstable proteins (Manilal et al., 1998b; Nagano et al., 1996), although other mutations can lead to changes in the subcellular localization of emerin and aberrant functions (Ellis et al., 1998).

Emerin is a tail-anchored membrane protein of 254 residues with a single predicted transmembrane domain close to its $\mathrm{C}$-terminal end and no signal peptide. Based on these topological features, the protein has been suggested to be post-translationally inserted into the cellular membrane system (Ellis et al., 1998). Such a mechanism, which is distinct from the classic pathway for signal recognition particle (SRP)-dependent insertion, was originally postulated for proteins whose C-terminal transmembrane domain cannot emerge from the ribosome before termination of translation (Kutay et al., 1993). A bioinformatics approach yielded $\sim 400$ potential human tail-anchored proteins (Kalbfleisch et al., 2007). Most tail-anchored proteins are thought to integrate into the ER membrane, although targeting to other organelles (e.g. peroxisomes or mitochondria) is possible (Hegde and Keenan, 2011). For insertion into the ER, conserved machinery has been identified in yeast and in mammalian cells. Important players are TRC40 [transmembrane domain recognition complex protein of $40 \mathrm{kDa}$, also known as ASNA1; guided entry of tail-anchored proteins 3 (Get3) in yeast], a protein that associates with the hydrophobic stretch of amino acids at the C-terminus of tail-anchored proteins (Favaloro et al., 2008; Stefanovic and Hegde, 2007), WRB (tryptophan-rich basic protein; Get1 in yeast) (Vilardi et al., 2011) and the mammalian-specific protein CAML $\left[\mathrm{Ca}^{2+}\right.$-modulating cyclophilin ligand, also known as CAMLG (Yamamoto and Sakisaka, 2012)]. Together, WRB and CAML function as the TRC40 receptor at the ER membrane (Vilardi et al., 2011, 2014; Yamamoto and Sakisaka, 2012). Furthermore, chaperone-like 
components, such as SGTA and BAG6, capture the C-terminal transmembrane domains as they emerge from the ribosome and then deliver tail-anchored proteins to TRC40 (Leznicki et al., 2010; Mariappan et al., 2010). However, a subset of tail-anchored proteins seems to be targeted to membranes independently of the TRC40 pathway (Rabu et al., 2008) and cytochrome $b 5$, for example, can be integrated into ER membranes in an unassisted manner (Colombo et al., 2009).

After membrane integration into the ER, emerin has to find its way to its final destination, the INM (Manilal et al., 1998a). Compared to nuclear import of soluble proteins, targeting of proteins to the INM is not well characterized (Burns and Wente, 2012; Ungricht and Kutay, 2015; Zuleger et al., 2012). Soullam and Worman (1995) identified specific INM-targeting signals within a nuclear region of the lamin B receptor (LBR), which comprises eight predicted transmembrane domains. Furthermore, the size of the nuclear region of the protein was suggested as a limiting factor for efficient transport to its final destination. Very recently, models that posit diffusion and retention as major determinants of INM localization of proteins have gained a lot of support (Boni et al., 2015; Ungricht et al., 2015). An energy requirement that had originally been observed for transport of proteins to the INM (Ohba et al., 2004) was attributed to ATP-dependent changes of ER structures that affect the diffusional mobility of proteins within the membrane system (Ungricht et al., 2015). In line with this interpretation, localization of emerin to the INM depends on its interaction with A-type lamins (Vaughan et al., 2001) and/or nesprins (Wheeler et al., 2007). As a result of retention at its binding site, the mobility of emerin at the INM is significantly reduced compared to that of overexpressed emerin localizing to the ER (Östlund et al., 1999). A subset of proteins, however, might also use active import pathways for transport to the INM (King et al., 2006; Kralt et al., 2015; Laba et al., 2015).

Besides the INM, emerin has been reported to localize to the peripheral ER and to the ONM, where it has been found to associate with the centrosome (Salpingidou et al., 2007), and to the plasma membrane, for example in adherens junctions of intercalated disks of cardiomyocytes (Cartegni et al., 1997). Despite this very diverse intracellular localization pattern of emerin, rather little is known about its mechanisms of membrane integration and subcellular trafficking. Early experiments with in-vitro-translated emerin suggested that the protein can indeed be post-translationally inserted into microsomal membranes (Ellis et al., 1998). In our study, we now provide evidence for a role of the TRC40 system in the post-translational ER membrane integration of emerin, and we analyze several emerin mutants associated with EDMD with respect to TRC40 binding, membrane integration and targeting to the INM.

\section{RESULTS}

\section{Post-translational membrane integration of emerin by the TRC40 pathway}

Emerin has been suggested to use the TRC40 pathway for membrane integration of tail-anchored proteins (Laba et al., 2014), but experimental evidence has not been available. Very recently, the crystal structure of the C-terminal transmembrane domain of the yeast tail-anchored protein Pep12 in a complex with its targeting factor Get3 has been solved (Mateja et al., 2015). The structure revealed a hydrophobic groove formed by a Get3 homodimer that shields the hydrophobic transmembrane domain of its substrate. We compared the properties of the transmembrane domains of Pep12 and emerin using a helical wheel projection (http://emboss.bioinformatics.nl/cgi-bin/emboss/ pepwheel; Fig. 1A). Strikingly, six out of 20 of the characteristic amino acid residues of the transmembrane domains are identical. In addition to the typical tail-anchored protein topology, this suggests that emerin might use the mammalian homologue of Get3, TRC40, to assist in post-translational membrane integration. We therefore used a system for the purification of tail-anchored proteins in a complex with TRC40, shielding the hydrophobic transmembrane domain and protecting the tail-anchored protein from aggregation. Emerin was expressed in bacteria as a fusion protein carrying an $\mathrm{N}$-terminal HZZ tag (i.e. a His tag linked to an IgG-binding ZZ-domain) and a C-terminal N-glycosylation site (an 'opsin' tag), together with maltose-binding protein (MBP)tagged TRC40, and purified using Ni-NTA- and amylose beads (Fig. 1B). Typically, this strategy yielded $\sim 0.3-1.2 \mathrm{mg}$ of wildtype protein per liter of bacterial culture, and the two proteins co-purified perfectly. This result demonstrates that emerin can indeed form stable complexes with TRC40.

Post-translational membrane integration of in-vitro-translated emerin has previously been addressed (Ellis et al., 1998). In that study, microsomal-membrane-associated emerin was sensitive to protease treatment, suggesting a type II orientation of the protein with its short C-terminal region behind the transmembrane domain facing the lumen of the microsomes. Protection of this short peptide from proteolysis, however, was not demonstrated (Ellis et al., 1998), presumably due to its small size. To unequivocally confirm post-translational membrane integration of emerin leading to a lumenal C-terminus of the protein, we took advantage of an assay where an $\mathrm{N}$-glycosylation site (opsin tag) fused to the $\mathrm{C}$ terminus of the protein of interest can be modified by lumenal glycosyl transferases (Pedrazzini et al., 2000). The purified MBPTRC40 and HZZ-emerin-opsin complex was incubated with rough microsomes from dog to assess post-translational membrane integration. A shift in molecular mass indicates N-glycosylation of the reporter protein, a reaction that is specific for the ER or, in this assay, the microsomal lumen. As shown in Fig. 1C, the addition of microsomes to the reaction resulted in a significant portion of a slower migrating form of emerin as compared to a reaction lacking microsomes, indicating post-translational modification(s). Furthermore, we observed a smear of high molecular mass forms of emerin. When the sample was treated with endoglycosidase $\mathrm{H}$ (EndoH) after the reaction, a slow-migrating form of emerin was partially lost and the faster migrating form became more prominent, demonstrating that the protein had originally been $\mathrm{N}-$ glycosylated. Consequently, the opsin tag with its N-glycosylation site must have reached the microsomal lumen, confirming membrane insertion of the tail-anchored protein. Addition of lambda-phosphatase largely abolished the smear of higher molecular mass forms, in agreement with earlier observations that emerin can be phosphorylated (Ellis et al., 1998; Leach et al., 2007; Roberts et al., 2006). TRC40-dependent membrane integration of tail-anchored proteins is an active, ATP-dependent process (Favaloro et al., 2008, 2010; Stefanovic and Hegde, 2007). Accordingly, the portion of the slower migrating form of emerin was clearly reduced when ATP was omitted from the reaction (Fig. 1D,E). We also performed reactions with microsomes that had been treated with trypsin to remove potential receptor proteins from the membranes. In these assays, the fraction that shifted in molecular mass was clearly reduced, suggesting that membrane insertion of emerin occurred in a receptor-dependent manner (data not shown). Finally, the addition of fragments of the TRC40receptor proteins WRB or CAML to the reaction almost completely prevented the shift in molecular mass (Fig. 1D,E), suggesting that 
the fragments function as dominant-negative inhibitors of TRC40mediated membrane integration, as shown previously for other tailanchored proteins (Vilardi et al., 2011; Yamamoto and Sakisaka, 2012).

The system described above uses emerin in a pre-assembled complex with TRC40. To analyze the TRC40-dependent membrane integration of emerin in a less biased manner, we took advantage of a coupled in vitro transcription-translation system. As shown in Fig. 2A, emerin-opsin was synthesized in vitro in a plasmiddependent manner. In the presence of rough microsomes, a shift in molecular mass was observed, which could be reversed by treating the reaction product with EndoH. Thus, the in vitro system
A

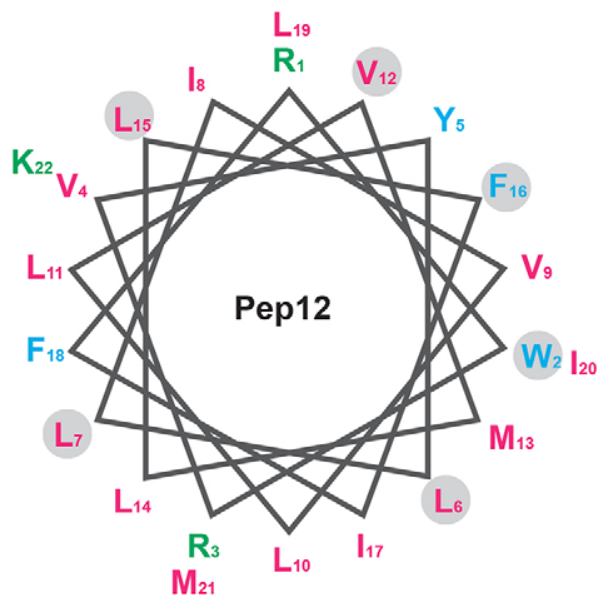

B

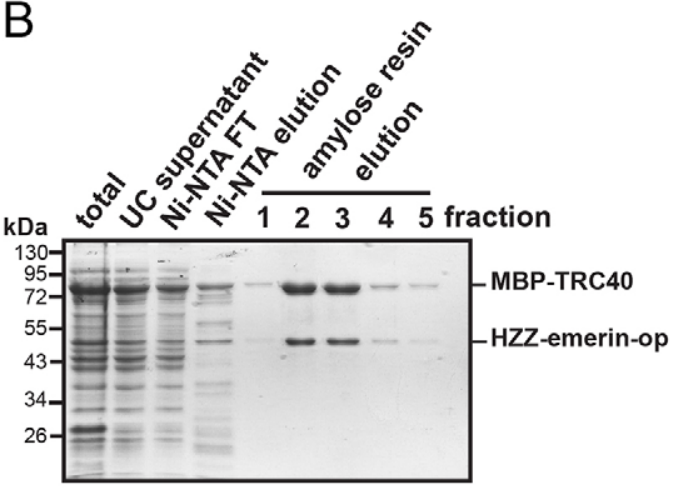

D

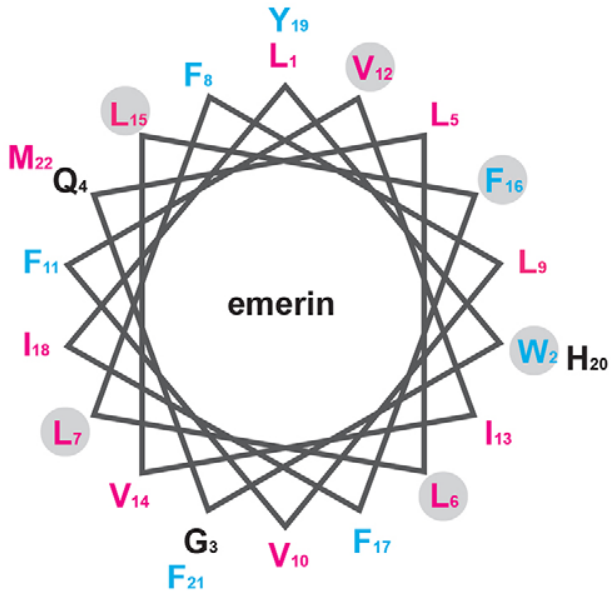

C

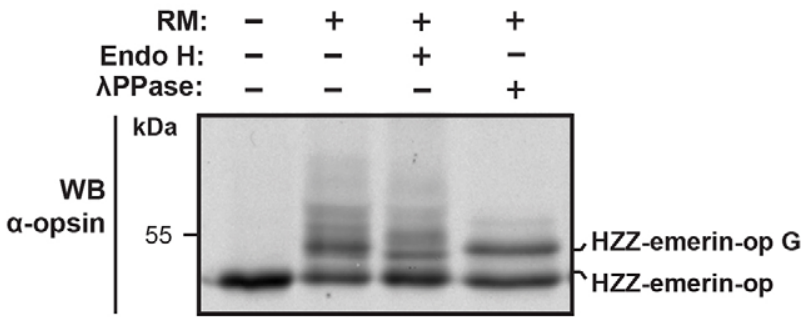

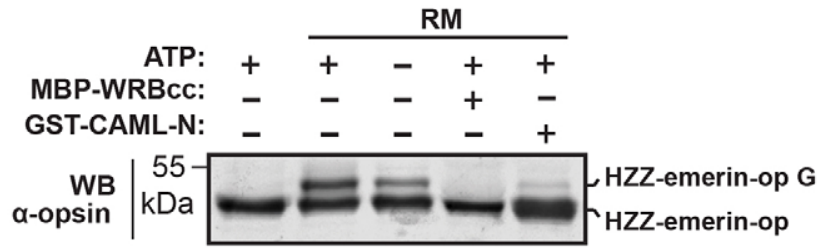

$\mathrm{E}$

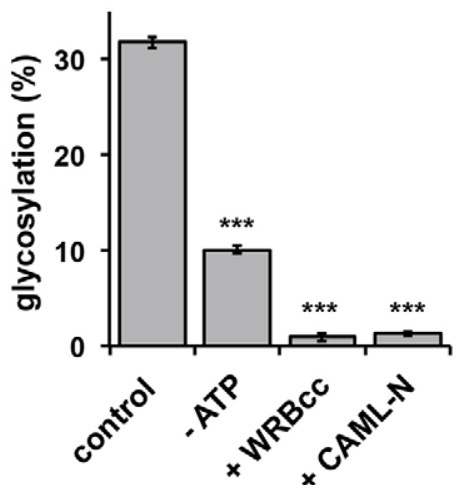

Fig. 1. The TRC40-pathway mediates the insertion of emerin into ER-enriched membranes. (A) Helical wheel projections of transmembrane domains of Pep12 and emerin. Hydrophobic residues are colored in magenta, aromatic residues in light blue and positively charged residues in green. The gray circles indicate identical residues between Pep12 and emerin. (B) Co-purification of the recombinant MBP-TRC40 and HZZ-emerin-opsin (op) complex from E. coli by Ni-NTA and amylose resins. UC, ultracentrifugation; FT, flow-through. (C) In vitro insertion of HZZ-emerin-op into rough microsomes (RM). The MBP-TRC40 and HZZ-emerin-opsin complexes were incubated with (+) or without $(-)$ RM. Where indicated, EndoH or lambda phosphatase ( $\lambda$ PPase) treatments were carried out after integration. WB, western blotting; $\mathrm{G}$ indicates the glycosylated form. (D) Effects of ATP depletion and WRBcc or CAML-N fragments (10 $\mu M$ each) on membrane integration of HZZ-emerin-opsin. (E) Quantification of relative amounts of glycosylated (i.e. membrane inserted) emerin-opsin under different conditions as in D. Error bars indicate the s.d. of four independent experiments. ${ }^{*} P<0.05 ;{ }^{* * *} P<0.001$ (Student's $t$-test). 
A

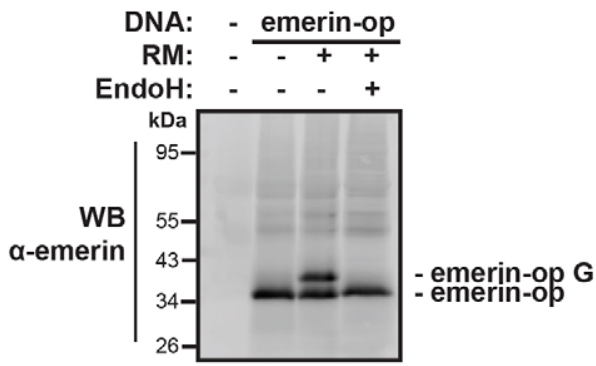

B

$\frac{\text { TRC40 }}{-\quad+} \frac{\text { HSC70 immunodepletion }}{-+}$

C

mock TRC40 $\underline{\mathrm{HSC} 70}$ immunodepletion

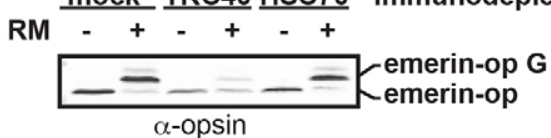

Fig. 2. In vitro translation and TRC40-dependent membrane insertion of emerin. (A) Opsin-tagged emerin (emerin-op) was produced in vitro by coupled transcription-translation in the absence $(-)$ or presence $(+)$ of rough microsomes (RM) and with (+) or without (-) subsequent treatment with EndoH. (B) Lysates used for coupled transcription-translation reactions were immunodepleted using antibodies against TRC40 or HSC70. (C) Depleted lysates (mock, anti-TRC40 and anti-HSC70 antibodies) were used for reactions as in A. In A-C, proteins were analyzed by SDS-PAGE, followed by immunoblotting (WB) using appropriate antibodies, as indicated. $G$ indicates the glycosylated form.

recapitulates microsome-dependent $\mathrm{N}$-glycosylation of the reporter protein and, hence, membrane integration, similar to the assay presented in Fig. 1. Next, we depleted the reticulocyte lysate that was used for in vitro translation using specific antibodies against TRC40 or, as a control, the chaperone HSC70 (also known as HSPA8) (Fig. 2B). Strikingly, the depletion of TRC40 from the lysate resulted in a strong reduction of $\mathrm{N}$-glycosylation (i.e. membrane integration) of in-vitro-translated emerin compared to mock-treated or HSC70-depleted lysates (Fig. 2C). Taken together, these results show that opsin-tagged emerin can be posttranslationally inserted into microsomal membranes in a TRC40-, WRB- and CAML-assisted manner.

Next, we set out to establish a membrane-integration assay for emerin in permeabilized cells. HeLa cells were treated with digitonin to preferentially permeabilize the plasma membrane and were then incubated with the purified MBP-TRC40 and HZZemerin-opsin complex. Similar to dog microsomes, the permeabilized cells were able to post-translationally insert emerin into ER membranes in an ATP- and temperature-dependent manner, as concluded from the observed shift in the molecular mass of the reporter protein. Again, insertion could be suppressed with WRB or CAML fragments, indicating a role for the TRC40 system (Fig. 3A). To confirm N-glycosylation as the basis for the observed shift in molecular mass, we treated the permeabilized cells with Peptide-Nglycosidase F (PNGaseF) after the first reaction. Similar to our observation with EndoH (Fig. 1C), this treatment resulted in a complete loss of the slowly migrating form of emerin with a concomitant increase in intensity of the faster migrating (i.e. deglycosylated) form (Fig. 3B).

Taken together, these results suggest post-translational membrane insertion of emerin through the TRC40-WRB-CAML system under conditions where emerin is presented to the permeabilized cells or microsomes as a preformed TRC40 complex (Figs 1 and 3) or expressed in an in vitro system (Fig. 2). Next, we asked whether interactions of emerin with the TRC40 system could be detected in situ, and we used a proximity ligation (Duolink) assay (PLA) (Söderberg et al., 2006) to address this question. This assay is based on the decoration of proteins present in a complex or in close vicinity in fixed cells with specific primaryand oligonucleotide-linked secondary antibodies. If the target proteins are in close proximity, subsequent ligation and amplification reactions lead to a fluorescent product that can easily be detected by microscopy (Söderberg et al., 2006; Fig. 4A). To demonstrate the suitability of our anti-emerin antibodies, we first set out to detect the well-established interaction of emerin with
A

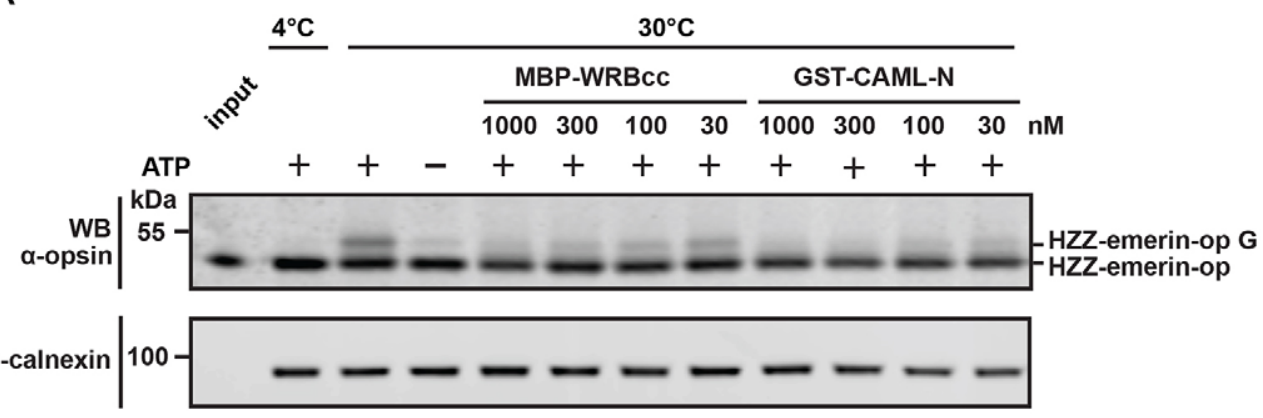

B

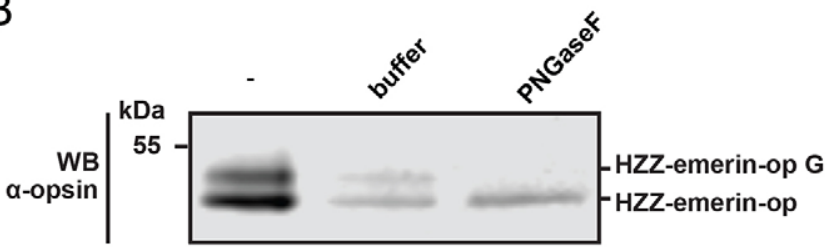

Fig. 3. Post-translational insertion of emerin into membranes of semipermeabilized cells. (A) HeLa cells were permeabilized with digitonin and incubated with purified $\mathrm{HZZ}$-emerinopsin (op) and MBP-TRC 40 at $4^{\circ} \mathrm{C}$ or $30^{\circ} \mathrm{C}$, with or without energy (+/- ATP) and increasing amounts of MBP-WRBcc or GST-CAML-N, as indicated. (B) After a reaction as in $A$ at $30^{\circ} \mathrm{C}$ in the presence of ATP, portions of the reaction were left untreated $(80 \%,-)$ or further incubated with (PNGaseF) or without (buffer; $10 \%$ each) the PNGaseF. In A and B, proteins were analyzed by SDS-PAGE, followed by immunoblotting (WB) using the indicated primary antibodies. Calnexin served as a loading control. $G$ indicates the glycosylated form of emerin. 
A

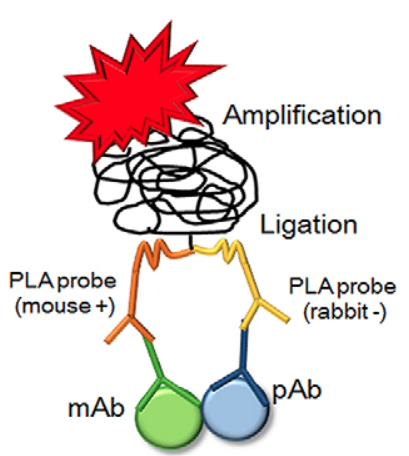

C

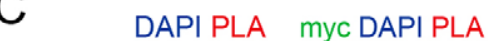

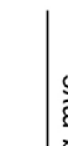
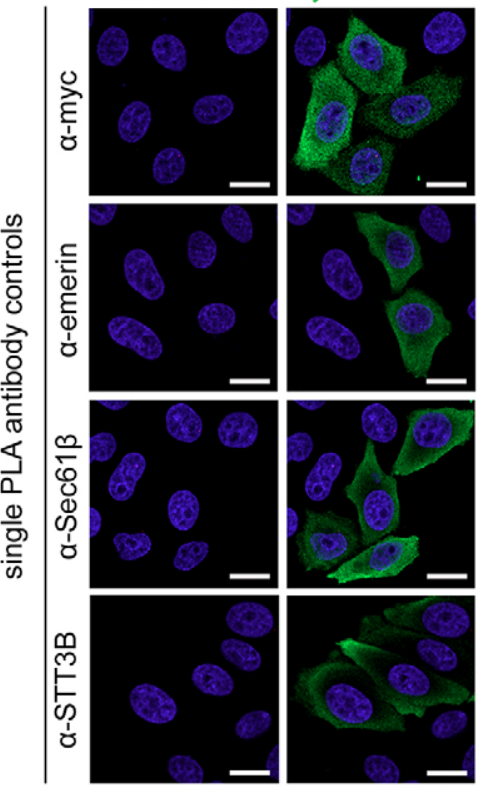

D

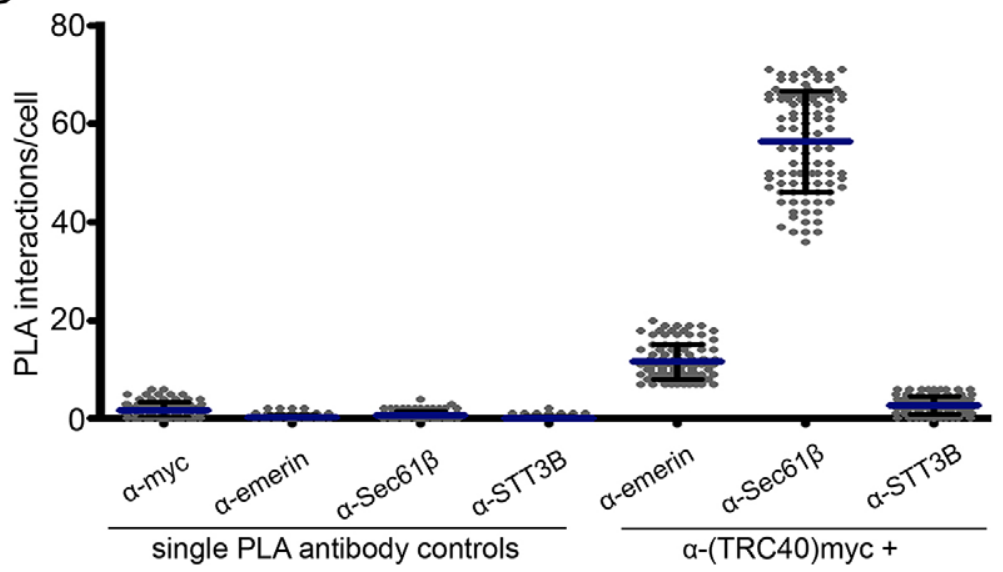

Fig. 4. In situ PLA detection of TRC40-emerin interactions. (A) Schematic diagram of the in situ PLA strategy showing primary antibodies and PLA probes binding to target proteins. Close proximity of the secondary PLA probes allows ligation followed by rolling circle amplification. (B) Intracellular localization of emerin and lamin $\mathrm{A} / \mathrm{C}$ complexes using the Duolink in situ PLA assay in HeLa cells. PLA signals of single proteins (emerin or lamin) or protein-protein complexes (emerin-lamin) are displayed. Each red dot represents a single protein-protein interaction. Nuclei were stained with DAPI (blue) and representative images are shown. (C) HeLa cells were transfected with a plasmid coding for TRC40-Myc prior to staining with anti-Myc, -emerin, -Sec61 $\beta$ (positive control) or -STT3B (negative control) antibodies to detect interactions using the PLA. Cells were then stained for TRC40-Myc (green) and DAPI (blue). Left panel, negative controls, with only one primary antibody for the targeted protein-protein interaction. Right panel, dual antibody PLA. Representative confocal images are shown. Scale bars: $10 \mu \mathrm{m}$. (D) The dot plot represents the number of PLA dots per cell for both the single antibody controls and proteinprotein interactions with mean values (blue bars) and s.d. (error bars) shown. 100 cells were scored over two independent experiments. lamins at the INM (Vaughan et al., 2001; Fig. 4B). After fixation and permeabilization, cells were treated with antibodies against endogenous emerin and/or lamin $\mathrm{A} / \mathrm{C}$ and subjected to the Duolink assay. After the reaction, red dots indicate sites where two proteins exist in proximity. When both antibodies were applied together, essentially all cells showed red fluorescent signals exclusively at the nuclear rim, indicating the interaction of emerin and lamin $\mathrm{A} / \mathrm{C}$ at the INM. No signals were detected when only one of the primary antibodies was used, demonstrating the specificity of the reaction
(Fig. 4B). Next, cells were transfected with Myc-tagged TRC40 and subjected to PLAs using antibodies against the Myc tag and different endogenous membrane proteins: (1) Sec61 $\beta$, an established tail-anchored protein and model protein used in studying the TRC40 system (Favaloro et al., 2008; Stefanovic and Hegde, 2007); (2) STT3B, an oligosaccharyltransferase that should integrate into ER membranes with the help of the SRP; and (3) emerin. Myc-TRC40 was found in cytoplasmic regions and largely excluded from the nucleus. As shown in Fig. 4C,D, no dots were 
detected in cells that had been treated with either one of the individual antibodies alone. A strong signal ( $~ 56$ dots per cell) was observed with antibodies against Sec61 $\beta$, our positive control, and the Myc-TRC40. The negative control, STT3B, yielded only very few dots per cell. Clearly, a specific interaction of emerin with MycTRC40 could be observed with an average of 12 red dots per transfected cell. For both Sec61 $\beta$ and emerin, the dots were mostly excluded from the nuclear region of the cells, further demonstrating the specificity of the detection.

In summary, these results show that transient interactions of emerin with TRC40 can be detected in situ and strongly suggest a physiological role for the TRC40 system in membrane integration of this tail-anchored protein. To further establish such a role in living cells, we established conditions for small interfering RNA (siRNA)mediated knockdown of TRC40 and analyzed targeting of endogenous emerin to the nuclear envelope in knockdown cells and in control cells. As a control, we detected the INM protein LBR (Soullam and Worman, 1995). LBR is co-translationally inserted into the ER membrane and, hence, does not require the TRC40 pathway. Treatment of cells with specific siRNAs resulted in a clear reduction of TRC40 levels, as detected by indirect immunofluorescence (Fig. 5A,B) or western blotting (Fig. 5C). Inspection of emerin levels at the nuclear envelope revealed a clear reduction of the protein in TRC40-knockdown cells compared to control cells (Fig. 5A,B), whereas the total levels remained unchanged (Fig. 5C). LBR and STT3B, by contrast, were not affected by the siRNAs (Fig. 5A,B). These results show that targeting of emerin to the cellular membrane system is reduced in TRC40depleted cells and they suggest that the non-targeted protein does not give rise to a strong signal in immunofluorescence microscopy.

\section{Membrane integration of EDMD mutants of emerin}

Having established the TRC40 pathway as a major route in posttranslational membrane integration of emerin, we next analyzed the role of the C-terminal region of emerin in detail. Several mutations within the transmembrane domain or in its close proximity have been associated with EDMD (Ellis et al., 1998; Manilal et al., 1998b; Mora et al., 1997; Nagano et al., 1996; Nigro et al., 1995; Ognibene et al., 1999; Vohanka et al., 2001; Yates et al., 1999). We selected several emerin mutants with increasing levels of expected severity (i.e. from point mutants with single amino acid changes to mutants lacking the entire transmembrane domain; Fig. 6A). The Gln228His mutant was originally identified in a patient with $\mathrm{X}$-linked mental retardation (Tarpey et al., 2009) and has not been linked to EDMD, and serves as a control for our analyses. A hydrophobicity plot of the Cterminal region of wild-type emerin and these mutants confirmed the expected changes (Fig. 6B): for the Pro183Thr, the Pro183His and the Gln228His mutant, the plot was almost identical to that of wild-type emerin. The stop codon mutation Trp226* (i.e. translation terminates after Leu225) and the frame shift mutation Leu225Arg-FS essentially abolished the transmembrane domain, whereas the Phe240His-FS mutation and the deletion $\Delta$ Val236Phe241 resulted in shorter hydrophobic domains of the corresponding proteins. For the latter two proteins and for wildtype emerin, we also predicted $\Delta \mathrm{G}$-values for insertion of putative transmembrane helices into membranes (Hessa et al., 2007). The $\Delta \mathrm{G}$-prediction server (http://dgpred.cbr.su.se/index.php? $\mathrm{p}=$ home) yielded values of $-4.288,-1.791$ and $0.642 \mathrm{kcal} / \mathrm{mol}$ for wildtype emerin, emerin-Phe240His-FS and emerin- $\Delta$ Val236-Phe241, respectively, confirming that these mutations result in less hydrophobic transmembrane domains.

Next, we co-expressed MBP-TRC40 and wild-type (compare Fig. 1B) or mutant forms of His- and opsin-tagged emerin in bacteria (Fig. 6C). The three point mutants Pro183Thr, Pro183His and Gln228His yielded similar levels of TRC40-HZZ-emerin complexes as the wild-type protein, whereas for emerin-Trp226*, Leu225Arg-FS and Phe240His-FS, as well as the deletion $\Delta$ Val236-Phe241, substantially less HZZ-emerin-opsin was recovered during the purification, indicating reduced interaction
A
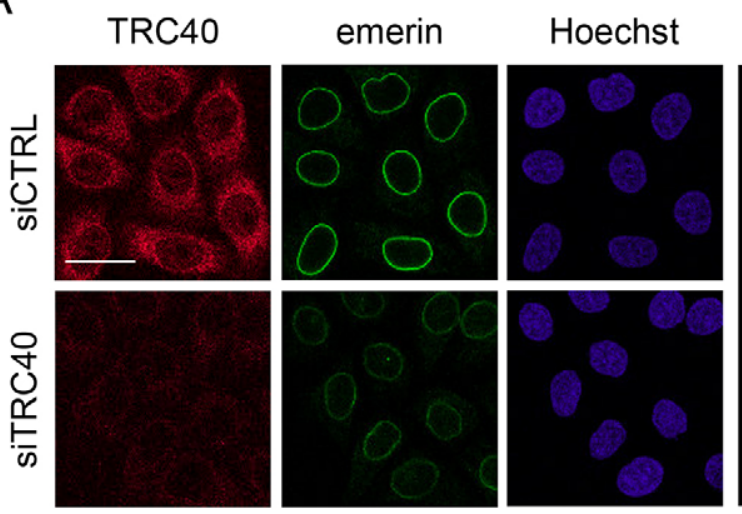

B

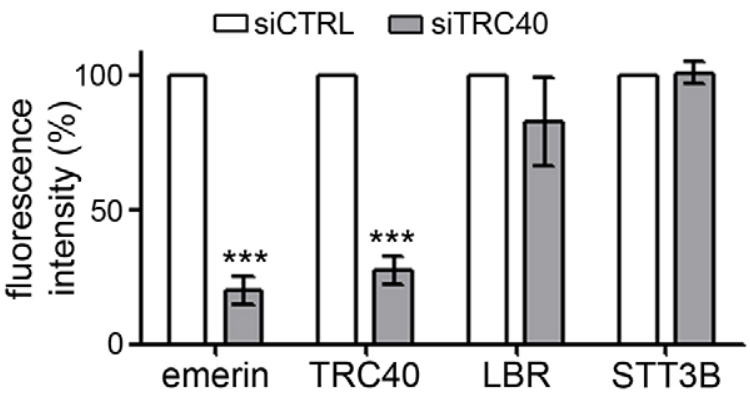

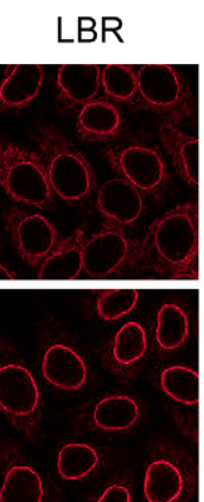

STT3B

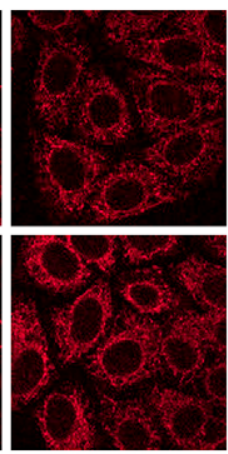

C

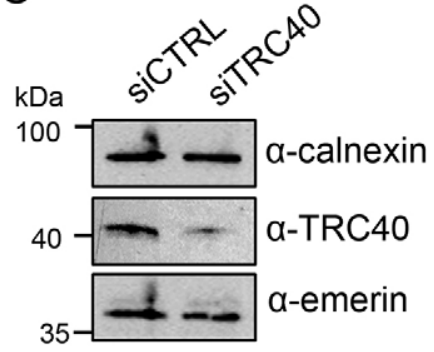

Fig. 5. Depletion of TRC40 results in reduced levels of emerin at the nuclear envelope. (A) HeLa cells were treated with a final concentration of $170 \mathrm{nM}$ of specific siRNAs to reduce the levels of endogenous TRC40 (siTRC40) or control siRNAs (siCTRL) and subjected to indirect immunofluorescence detecting TRC40 and emerin or, on separate slides, LBR and STT3B, as indicated. Scale bar: $20 \mu \mathrm{m}$. Very similar results were obtained with siRNA concentrations as low as $50 \mathrm{nM}$.

(B) Quantification of the results in A. Error bars represent the s.d. from the mean fluorescence values from 100 cells over two independent experiments. ${ }^{* * *} P<0.001$ (Student's $t$-test). (C) Western blot of cell lysates of control cells or knockdown cells. Calnexin was used as a loading control. 
A

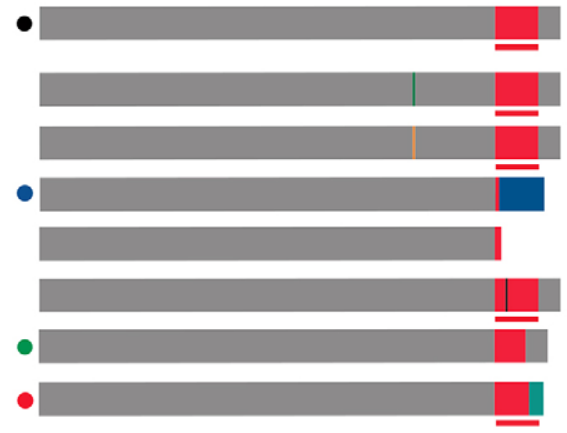

WT
VPLWGQLLLFLVFVIVLFF IYHFMQAEEGNPF
- VPRPAAAFPGLCDRPLLHLPLHAG
- VPLWGQLLLFLVF------IYHFMQAEEGNPF
- VPLWGQLLLFLVFVIVLHLPLHAG

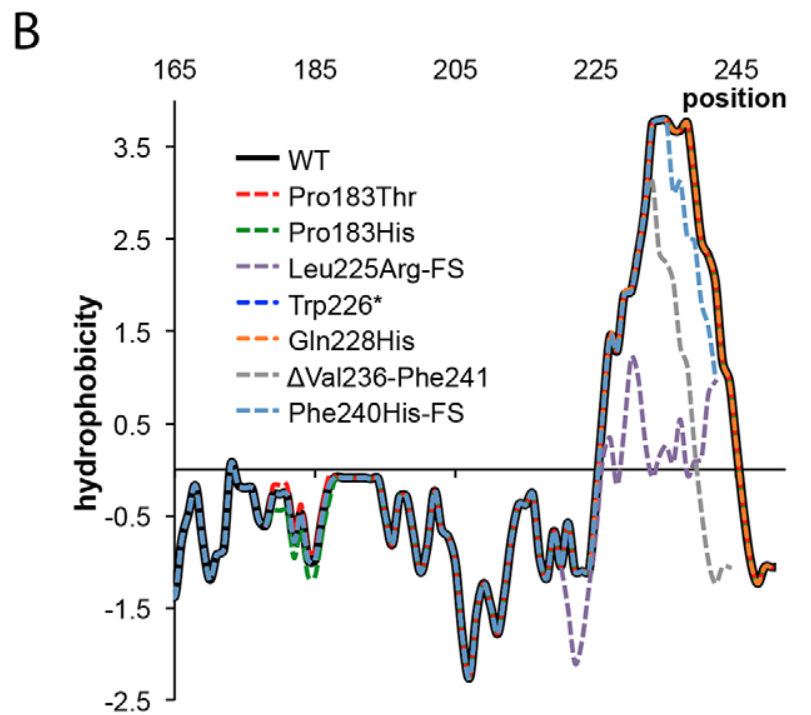

C

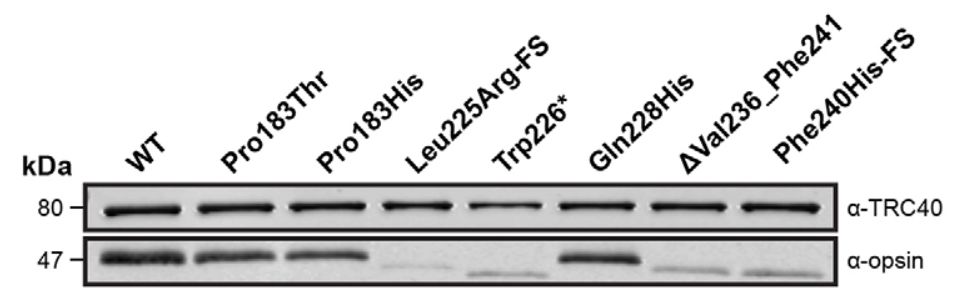

$\mathrm{D}$

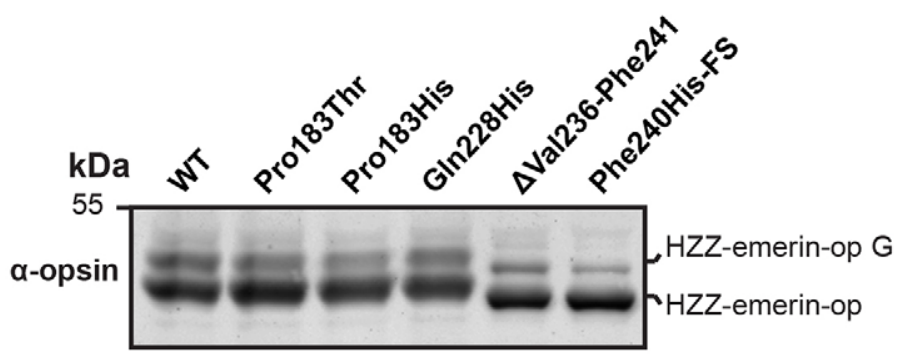

E

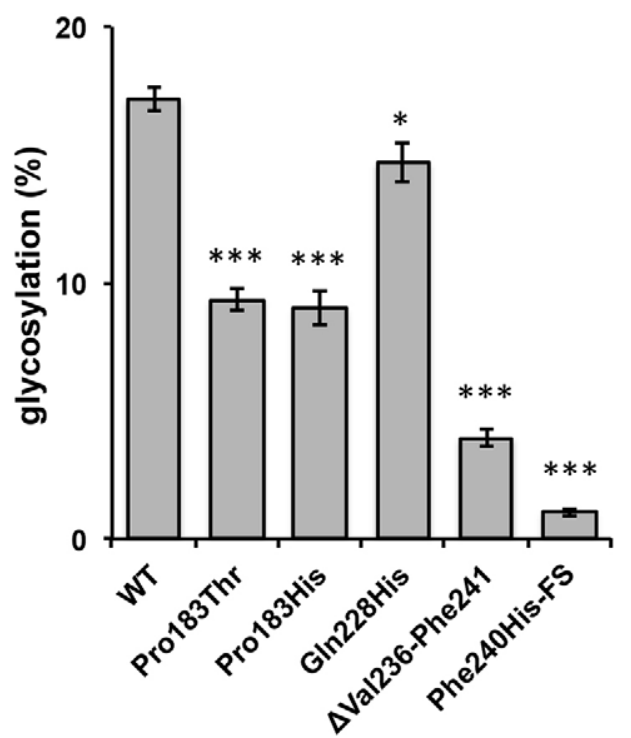

Fig. 6. Emerin disease mutants display impaired interaction with TRC40 and reduced membrane interaction. (A) Scheme of the emerin variants analyzed in this study. The transmembrane domain (TMD) is shown in red. Predicted TMDs in emerin variants are highlighted with a red line. TMDs of wild-type (WT) and Leu225Arg-FS, $\triangle$ Val236-Phe241, Phe240His-FS emerin variants are specified in detail below. (B) Kyte-Doolittle hydrophobicity plots for the C-terminal portion of the emerin mutants. (C) Co-purification of MBP-TRC40 and HZZ-emerin-opsin (op) complexes. Purified proteins were subjected to SDS-PAGE followed by western blotting detecting TRC40 and the opsin tag of the emerin variants. (D) In vitro insertion assay of HZZ-emerin-opsin variants into rough microsomes. Variants Trp226* and Leu225Arg-FS, which lack a transmembrane domain, were omitted from the analysis. G indicates the glycosylated form. (E) Quantification of the results in D. Relative amounts of glycosylated (i.e. membrane inserted) emerin mutants are depicted. Error bars indicate the s.d. of four independent experiments. ${ }^{*} P<0.05 ;{ }^{* \star *} P<0.001$ (Student's $t$-test).

with MBP-TRC40 (Fig. 6C). Equivalent amounts of purified emerin variants were then subjected to glycosylation assays using microsomal membranes. As shown in Fig. 6D,E, the Gln228His mutation hardly affected the degree of glycosylation (i.e. membrane integration), whereas for the Pro183Thr and Pro183His mutant, significantly less glycosylation was observed. The frame shift mutation Phe240His-FS and the deletion $\Delta$ Val236-Phe241 strongly reduced the efficiency of membrane integration of the respective proteins. These results show that TRC40-dependent membrane insertion of emerin can be affected by mutations within the transmembrane domain and, surprisingly, by single amino acid changes $\sim 40$ residues upstream of the transmembrane domain.

\section{Targeting of emerin and emerin mutants to the INM}

The most prominent localization of emerin is at the INM, although other localizations have been described. We therefore used transfection experiments and indirect immunofluorescence to compare the subcellular localization of our mutant forms of emerin with that of the wild-type protein. As expected, after fixation and Triton X-100 permeabilization of cells, HA-tagged wild-type emerin was predominantly found at the nuclear rim (Fig. 7). A similar pattern was observed for the three point mutants Pro183Thr, Pro183His and Gln228His, with some cell-to-cell variations regarding the level of extra-nuclear (i.e. presumably ERassociated) HA-emerin. The mutants Leu225Arg-FS and Trp226* 


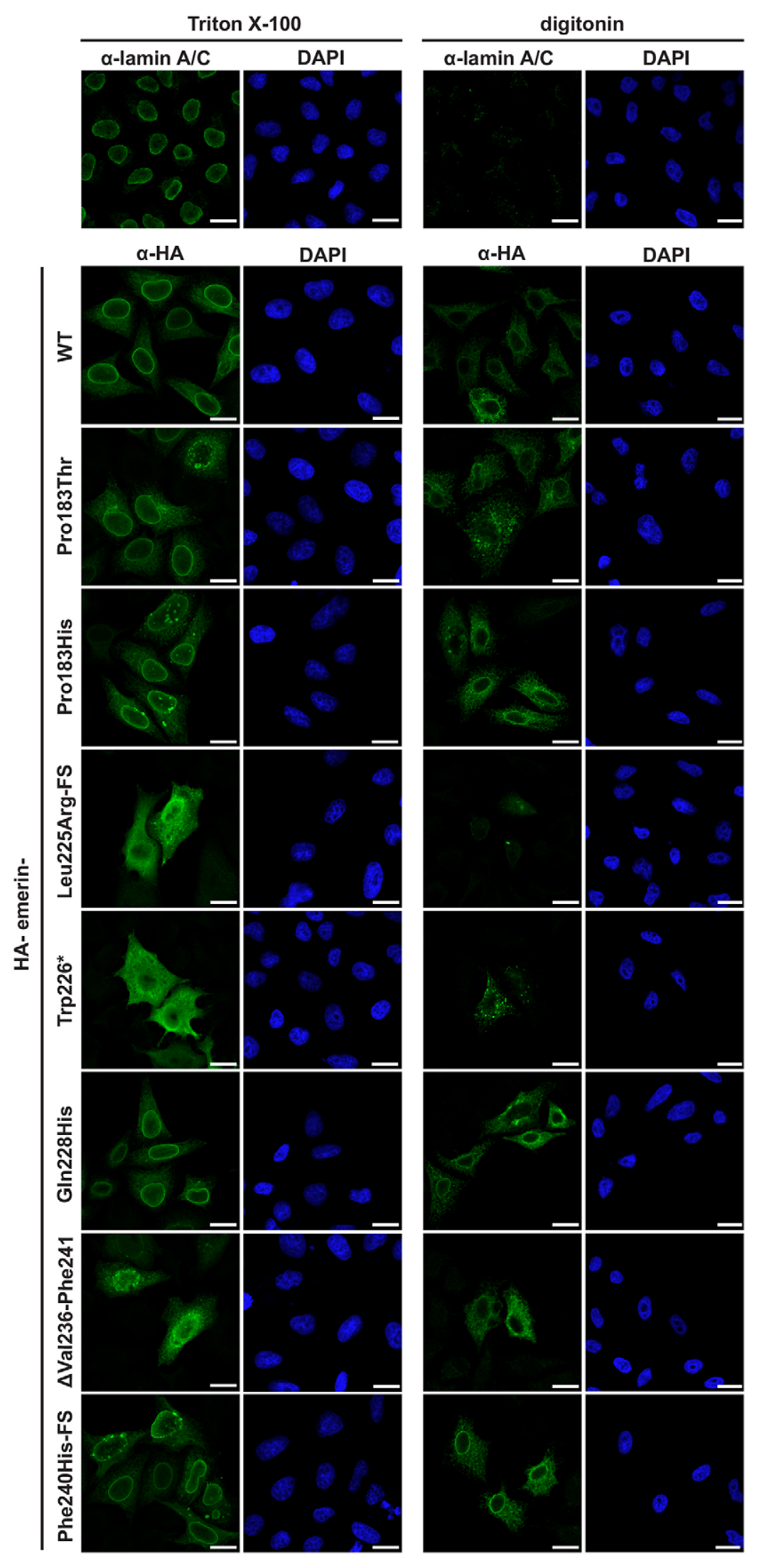

Fig. 7. Subcellular localization of emerin variants. HeLa cells were transfected with plasmids coding for HA-emerin variants, permeabilized either with Triton X-100 or digitonin and immunostained using antibodies against the $\mathrm{HA}$ tag.

Endogenous lamin A/C immunostaining (top row) was used as a control for differential permeabilization. Scale bars: $15 \mu \mathrm{m}$.

as well as the deletion $\Delta$ Val236-Phe241 showed almost no localization to the nuclear rim and were found all over the cells. Interestingly, the frame shift mutant Phe240His-FS, which hardly interacted with MBP-TRC40 (Fig. 6C) and was the least efficient in the membrane integration assay (Fig. 6D,E), was found at the nuclear rim, very similar to wild-type emerin. To discriminate 
between protein localization at the INM versus the ONM, we also performed immunostaining of cells subjected to differential permeabilization. Using the detergent digitonin, proteins of the INM should not be accessible to the primary antibody. Accordingly, our endogenous control protein of the INM, lamin $\mathrm{A} / \mathrm{C}$, was not detectable in digitonin-permeabilized cells, whereas a characteristic nuclear rim was observed after permeabilization with the detergent Triton X-100 (Fig. 7, top row). For HA-tagged wild-type emerin, a clear extra-nuclear signal was visible upon digitonin-treatment. Signal intensities at the nuclear rim, however, were reduced compared to Triton-X-100-treated cells, as expected. This result suggests that overexpressed HA-emerin partially reaches the INM and is partially sequestered at the ER and the ONM (as indicated by the digitonin experiment). For the Pro183 mutants and the Gln228His mutant, similar observations were made with clear, albeit weak signals, at the nuclear rim. The two emerin mutants lacking a transmembrane domain (Leu225Arg-FS and Trp226*) yielded only very weak signals in the digitonin-permeabilized cells, because the proteins are expected to be soluble and should be released from the cells upon treatment with the detergent. The deletion mutant $\Delta$ Val236-Phe241 exhibited similar signals upon Triton X-100 or digitonin treatment, without a pronounced staining at the nuclear rim. Strikingly, the emerin mutant Phe240His-FS appeared very similar in Triton-X-100- or digitonin-treated cells, with a clear nuclear rim under both conditions, suggesting that the protein localizes predominantly to the ONM. Taken together, our differential permeabilization experiments suggest that certain mutations in the emerin gene not only affect membrane integration of emerin but also its correct targeting to the INM.

To corroborate these results, we established an assay to specifically assess targeting of a reporter protein to the INM, similar to a previously published approach (Ohba et al., 2004). The system is based on induced dimerization of proteins carrying appropriate binding domains for rapamycin (Chen et al., 1995). Rapamycin binds to the $12-\mathrm{kDa}$ FK506 binding protein (FKBP12) as well as a 100-amino-acid domain of mammalian target of rapamycin (mTOR), known as the FKBP-rapamycin binding domain (FRB; Fig. 8A). Thus, rapamycin should recruit a soluble nuclear reporter protein containing an FKBP12-domain $\left(\mathrm{GFP}_{2}-\right.$ GST-NLS-FKBP12) to the INM if an FRB-containing protein (e.g. FRB-emerin) is present at this specific location. In our system, mCherry-FRB-emerin was expressed in cells together with $\mathrm{GFP}_{2}-$ GST-NLS-FKBP12, which showed a typical nuclear localization in the absence of rapamycin (Fig. 8B). Addition of rapamycin to the cells led to a very clear re-distribution of $\mathrm{GFP}_{2}-\mathrm{GST}-\mathrm{NLS}-$ FKBP12 to the nuclear periphery, resulting from drug-induced dimerization with the INM protein mCherry-FRB-emerin. As a control, we expressed WRB-FRB-HA together with the GFP reporter. At first inspection, WRB-FRB-HA showed a similar subcellular localization to mCherry-FRB-emerin, with staining in ER regions, and at the perinuclear ER and the nuclear rim. Addition of rapamycin, however, did not result in a redistribution of nuclear $\mathrm{GFP}_{2}-\mathrm{GST}-\mathrm{NLS}-\mathrm{FKBP} 12$, suggesting that WRB-FRB-HA is restricted to the ER and the ONM and absent from the INM (Fig. 8B).

Next, we used this assay to analyze INM-targeting of wild-type and mutant forms of emerin (Fig. 8C). For a better comparison of targeting efficiencies (Fig. 8D), the GFP $_{2}-$ GST-NLS-FKBP12 signal at the INM in rapamycin-treated cells was scored as ' +++ ', ' ++ ', '+' and '-' (see legend for details). The two mutants lacking a transmembrane domain, mCherry-FRB-emerin-Trp226* and -Leu225Arg-FS (Fig. 6A,B), localized to the nucleus, in agreement with previous studies, where similar constructs had been used (Östlund et al., 1999; Tsuchiya et al., 1999). Consequently, rapamycin did not induce a relocalization of the GFP reporter protein to the nuclear periphery - instead, this depended on the presence of an FRB-presenting protein at the INM (Fig. 8B). The deletion mutant mCherry-FRB-emerin- $\Delta$ Val236Phe241 was mostly found in aggregates throughout the cell and the co-expressed GFP reporter was not sequestered at the nuclear rim in the presence of rapamycin. The point mutants mCherry-FRBemerin-Pro183Thr, -Pro183His and -Gln228His, which all localized to the nuclear rim as HA fusion proteins in a very similar fashion to wild-type emerin (see Fig. 7), also showed a similar mCherry signal to the corresponding wild-type protein (Fig. 8C). Accordingly, rapamycin induced recruitment of the GFP reporter to the INM, albeit to a lower extent compared to cells that had been co-transfected with wild-type mCherry-FRB-emerin ('++' versus ' +++ '). Strikingly, reduced INM targeting was even more obvious for the frame shift mutant mCherry-FRB-emerinPhe240His-FS, which also showed a very prominent localization at the nuclear rim as an HA-tagged protein, irrespective of the permeabilization method (Fig. 7). Here, the effect of the rapamycin treatment was rather variable: in many cells $(56 \%, 100$ cells analyzed), rapamycin did not induce relocalization of $\mathrm{GFP}_{2}-\mathrm{GST}-$ NLS-FKBP12 to the nuclear rim at all. In others, the effect was rather subtle compared to cells expressing wild-type mCherryFRB-emerin, demonstrating that targeting to the INM was clearly impaired for this emerin mutant.

Thus, transport of emerin to or its retention at the INM depends on crucial features of the transmembrane domain or the luminal C-terminus and, potentially, on additional single amino acid residues in its close proximity. The assay shows that all disease mutants investigated reduce the fidelity with which emerin reaches its final destination, the INM. Localization at the nuclear rim, as observed for emerin mutants in previous studies (Fairley et al., 1999; Lee et al., 2001) is clearly not equivalent to correct targeting to the INM.

\section{DISCUSSION}

In this study, we analyzed membrane insertion and targeting of emerin to the INM and several clinically relevant emerin mutants linked to EDMD. The TRC40 pathway is the most prominent route for post-translational membrane integration of tail-anchored proteins, although other pathways that are promoted by HSC70 or HSP40 (Rabu et al., 2008) or the SRP (Abell et al., 2004, 2007) have been described. Furthermore, membrane insertion can occur through unassisted pathways that do not depend on additional soluble and/or membrane-bound factors (Brambillasca et al., 2006; Colombo et al., 2009). Upon co-expression in bacteria, emerin and TRC40 formed a stable complex (Fig. 1B), suggesting that emerin can also be captured by TRC40 as soon as it emerges from the translating ribosome in eukaryotic cells. Accordingly, depletion of TRC40 from reticulocyte lysates resulted in a clearly reduced membrane insertion of emerin (Fig. 2C). Given that the interaction of tail-anchored proteins with components of the TRC40 machinery is rather transient, it has been difficult to detect by classical coimmunoprecipitation approaches. However, PLAs corroborated our findings, showing that endogenous emerin interacts with overexpressed TRC40, albeit with lower signal numbers than endogenous Sec61 $\beta$. In this assay, the number of dots that are scored as single interaction sites depends on (among other factors) the abundance of the individual proteins and on the quality of the specific antibodies. Hence, a quantitative comparison of the 
A

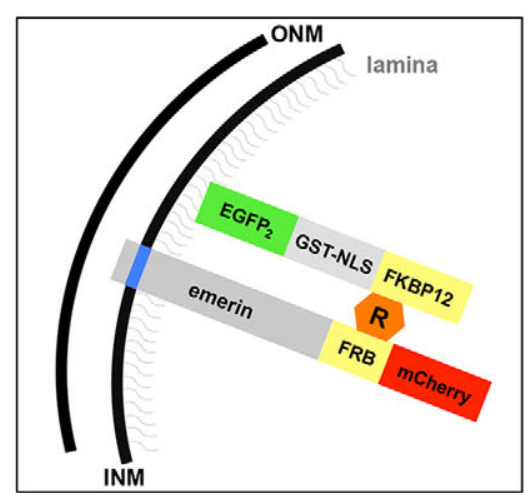

C
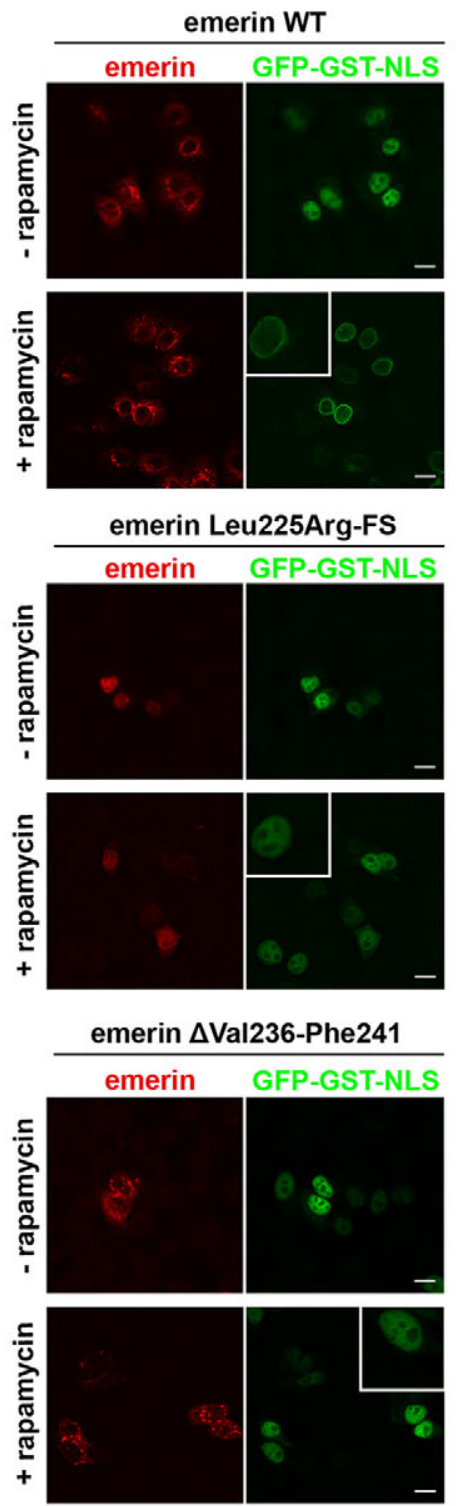

B
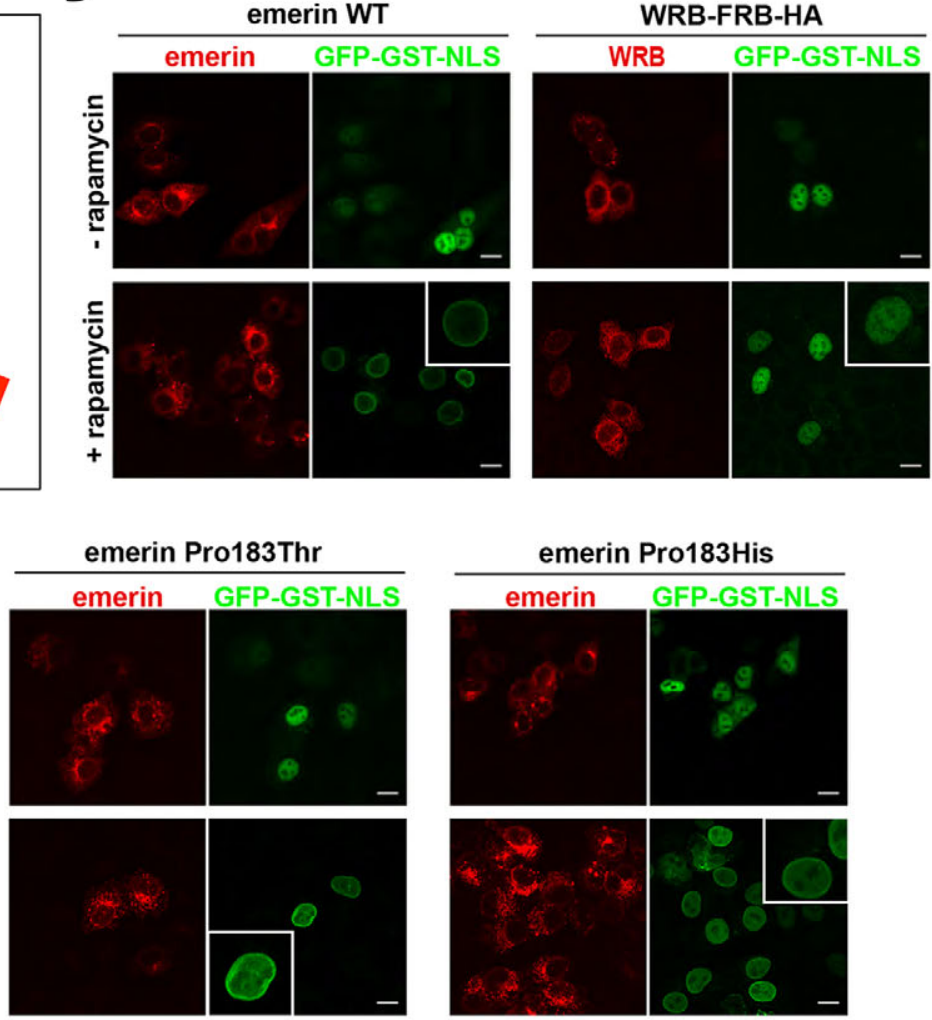

emerin Trp226*
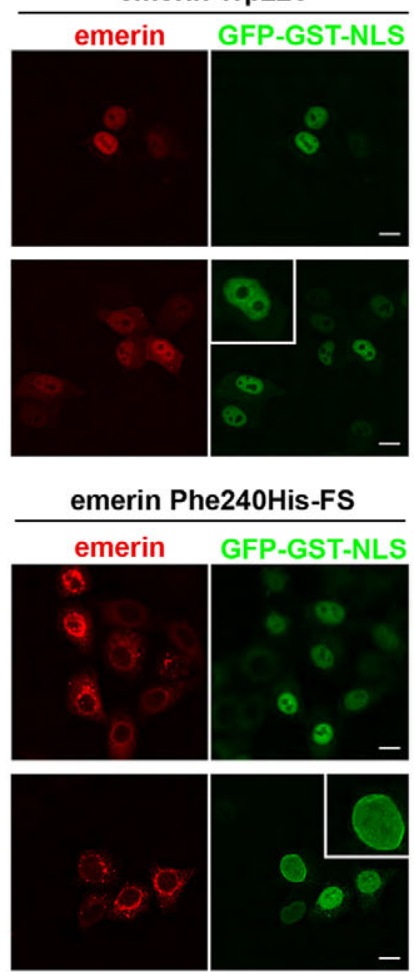
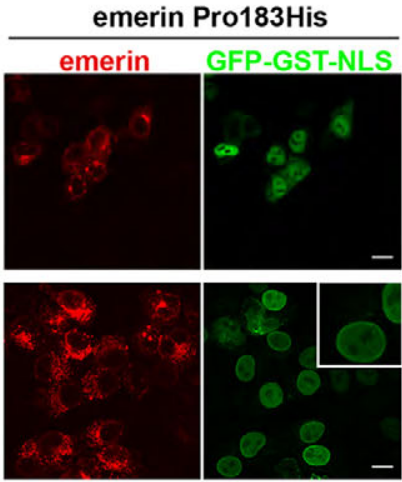

emerin GIn228His

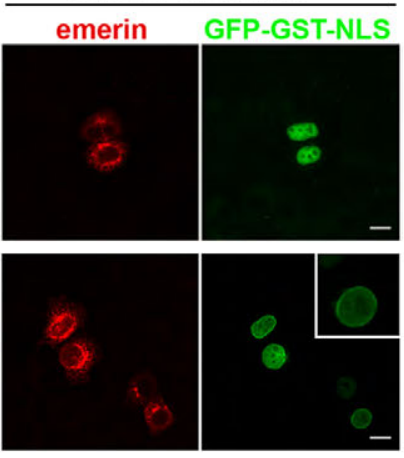

D $\begin{array}{cc}\begin{array}{c}\text { mCherry-FRB- } \\ \text { emerin }\end{array} & \text { GFP }_{2} \text {-GST-NLS-FKBP12, } \\ & \text { INM (+ rapamycin) }\end{array}$

\begin{tabular}{cc|}
\hline WT & ++ \\
\hline Pro183Thr & ++ \\
\hline Pro183His & ++ \\
\hline Leu225Arg-FS & - \\
\hline Trp226* & - \\
\hline Gin228His & ++ \\
\hline$\Delta$ Val236-Phe241 & - \\
\hline Phe240His-FS & + \\
\hline
\end{tabular}

Fig. 8. Mutations in the emerin gene affect targeting to the INM. (A) Rapamycin assay. Upon rapamycin (R) treatment, the soluble nuclear EGFP ${ }_{2}-G S T-N L S-$ FKBP12 reporter is recruited to mCherry-FRB-emerin at the INM. (B,C) HeLa cells were co-transfected with constructs coding for EGFP ${ }_{2}-G S T-N L S-F K B P 12$ (GFP-GST-NLS) and WRB-FRB-HA (WRB; B) or variants of mCherry-FRB-emerin (emerin; B,C), permeabilized with digitonin and treated with (+) or without (-) rapamycin. WT, wild-type. After fixation, cells were analyzed by confocal microscopy, either directly (emerin) or after immunostaining (WRB-FRB-HA). Scale bars, $15 \mu \mathrm{m}$. Typical cells at higher magnification (+ rapamycin) are shown in the inserts. Note that under our assay conditions (10 min on ice), the GFP reporter protein does not exit from the nucleus. (D) Semi-quantitative analysis of the rapamycin-effect on $\mathrm{EGFP}_{2}-\mathrm{GST}-\mathrm{NLS}-\mathrm{FKBP} 12$. +++, clear rim in essentially all cells; ++, visible rim in most cells but residual nuclear signal; +, visible rim in some cells, signal mostly nuclear; and -, no nuclear rim). Between 20 (Trp226*) and 100 cells (WT, Phe240His-FS) from separate experiments were scored. 
interaction of the two tail-anchored proteins with TRC40 is not possible. Nevertheless, for Sec61 $\beta$ as well as for emerin, the signals were specific and almost exclusively detected in the cytoplasmic region of the cells, consistent with interactions occurring at or close to ER membranes. PLAs have been used previously for the detection of TRC40 binding to tail-anchored proteins (Hradsky et al., 2011), but, to our knowledge, only upon overexpression of both interacting partners. Here, we used antibodies against endogenous emerin to monitor binding to lamin $\mathrm{A} / \mathrm{C}$ as well as Myc-tagged TRC40. Hence, this assay should be applicable for the analysis of interactions between other tail-anchored proteins and TRC40 or other components of the TRC40 machinery.

Our integration assays using microsomes (Figs 1, 2 and 6) or permeabilized cells (Fig. 3) clearly confirmed the possibility of post-translational membrane insertion of opsin-tagged emerin in a TRC40-, WRB- and CAML-assisted manner. The permeabilized cell system should be useful for future approaches, e.g. for analysis of membrane insertion upon knockdown of certain components of the TRC40 machinery. Our in vivo experiments with TRC40depleted cells (Fig. 5) further strengthen these findings. Of course, we cannot rule out the possibility that in cells lacking TRC40, membrane insertion of emerin is supported by alternative posttranslational mechanisms. In summary, we provide experimental evidence for emerin as the first tail-anchored protein of the INM that uses the TRC40 pathway for membrane insertion.

Several studies have analyzed the role of specific properties of individual transmembrane domains as well as of neighboring regions on post-translational membrane insertion of tail-anchored proteins (Beilharz et al., 2003; Borgese et al., 2001). As expected, emerin mutants with a complete loss of (Trp226*) or with drastic changes within the transmembrane domain (Leu225Arg-FS, $\Delta$ Val236-Phe241, Phe240His-FS) showed strongly reduced binding to TRC40 (Fig. 6C) and impaired membrane insertion in the microsome assay (Fig. 6C,D). Accordingly, HA-tagged emerin-Trp226*, -Leu225Arg-FS and - $\Delta$ Val236-Phe241 did not show a prominent localization at the nuclear envelope in transfection experiments (Fig. 7). Surprisingly, however, HA-emerinPhe240His-FS was found at the nuclear rim, very similar to the wild-type protein, although it showed strongly reduced insertion efficiency into ER-derived microsomes (Fig. 6D,E). Perhaps this mutant with its shortened transmembrane domain and a less negative $\Delta \mathrm{G}$-value for membrane insertion (-1.791 compared to $-4.288 \mathrm{kcal} /$ mol for wild-type emerin) is able to insert into the cellular membrane system in an unassisted manner or with the help of certain chaperones that are present in intact cells but not in the microsome assay. Similar results have been described for protein tyrosine phosphatase 1B, whose transmembrane domain is less hydrophobic than that of emerin (Brambillasca et al., 2006). Similarly, the emerin mutants Pro183Thr and Pro183His showed slightly reduced binding to TRC40 and significantly reduced membrane insertion (Fig. 6C,D), yet the localization of HA-tagged proteins appeared normal (Fig. 7). The transmembrane domains of these mutants are identical to that of wild-type emerin, suggesting that residues upstream of the hydrophobic stretch can affect the interaction with TRC40. For the corresponding GFP-tagged emerin mutants, reduced levels of fluorescence at the nuclear rim have been described previously (Fairley et al., 1999). Correct targeting to the INM, as opposed to the ONM was, however, not analyzed in that study. Our immunofluorescence results after differential permeabilization (Fig. 7) and the results of the rapamycin assay (Fig. 8C,D) now suggest that the emerin-Pro183 mutants and, in particular, the Phe240His-FS mutant have impaired targeting to the INM, although, at the level of light microscopy, their localization pattern at the nuclear envelope was similar to that of the wild-type protein.

The exact route of emerin (or other proteins of the INM) from the ER via the ONM and the NPC to the INM is not known. With respect to molecular mechanisms, two very recent publications (Boni et al., 2015; Ungricht et al., 2015) have shown that passive diffusion followed by subsequent retention at the target site are major determinants of INM localization. Nevertheless, energydependent changes in ER morphology have been suggested to affect the transport rates of proteins to the INM (Ungricht et al., 2015) and are also expected to affect proteins that are post-translationally inserted into the ER membrane. Selected proteins, by contrast, seem to require more active, transport-factor-dependent mechanisms (Laba et al., 2015). Transmembrane proteins that are cotranslationally inserted into the ER membrane must passage through the NPCs in a membrane-bound form, possibly through dedicated, peripheral channels (Maimon et al., 2012). For tailanchored proteins, it is principally possible that they are imported into the nucleus as soluble complexes with TRC40, followed by direct insertion into the INM from within the nuclear interior. In light of the low nuclear levels of TRC40 (Fig. 4C), the rather few PLA signals revealing emerin-TRC40 interactions inside the nucleus (Fig. 4C) and the apparent absence of the receptor protein WRB from the INM (Fig. 8B), this route seems rather unlikely. Instead, emerin is probably inserted into the ER system as a default membrane for tail-anchored proteins. From the ER, it is expected to traffic to the INM via the ONM and the NPC. Emerin as well as several other proteins of the INM contain putative nuclear localization signals (NLSs) and emerin fragments lacking the transmembrane domain were found to accumulate in the nucleus as a result of the NLS activity (Östlund et al., 1999; Tsuchiya et al., 1999). The significance of these NLSs and also of their cognate receptor proteins (e.g. importin $\alpha$ and $\beta$ ) for trafficking of membrane-bound proteins across the NPC, however, remains unclear.

In intact cells, the different mutant emerin variants that we tested reached the INM with efficiencies that cannot entirely be predicted by the efficiency of membrane insertion into microsomes. Thus, our results might suggest that membrane insertion of emerin through the TRC40 pathway and trafficking of the protein to the INM are sequential processes with distinct requirements. However, the major determinants for proper localization of emerin to the INM have been localized to regions outside of the transmembrane domain (Östlund et al., 1999; Tsuchiya et al., 1999). Our observations thus raise the interesting possibility that integration through the TRC40 pathway affects the correct targeting of emerin to its final destination, beyond initial membrane insertion. Perhaps the emerin mutant Phe240HisFS reaches the ONM by an alternative pathway that is not compatible with probing the INM and its constituent proteins. In summary, the molecular pathogenesis of EDMD as caused by the different mutations is likely to be complex. Damaging effects might result from reduced levels of functional emerin in the INM or from toxicity caused by mislocalized emerin variants. Given that interaction with nuclear proteins is key to emerin functions (Demmerle et al., 2012), mislocalization to the ONM or the ER is expected to contribute to the pathologic phenotypes of certain emerin mutants. Furthermore, mislocalization of tail-anchored proteins to the outer membrane of mitochondria has been recognized as a potential mechanism of toxicity in yeast and mammalian cells (Chen et al., 2014; Okreglak and Walter, 2014; Schuldiner et al., 2008). It will be necessary to consider these different possible causes of disease when further elucidating the 
targeting of wild-type emerin and its mutant variants in the pertinent differentiated cells, like skeletal muscle cells or cardiomyocytes.

\section{MATERIALS AND METHODS} Plasmids and constructs

All constructs were obtained using standard methods and verified by sequencing. The vector for coexpression of MBP-TRC40 and an HZZ-tagged tail-anchored protein was as described previously (Favaloro et al., 2010). Briefly, the coding sequence of human emerin was amplified by PCR using emerin-GFP (obtained from Eric Schirmer, Edinburgh, UK) as a template and forward (5'-TTTGGTACCACCATGGACAACTACGCAGATCTT- $3^{\prime}$ ) and reverse (5'-CAAGCTTTATCAGCCCGTCTTGTTGGAGAAAGGCACGTAGAAGTTTGGGCCGAAGGGGTTGCCTTCTTCAG-3') primers, the latter also coding for an opsin tag. The PCR-product was cloned into a modified pET-vector (pET328) through KpnI and HindIII, generating a fusion construct with an N-terminal HZZ tag (pET328-HZZ-Emerin-opsin). The modified emerin-sequence was then cut with AvrII and NheI and cloned into a pQE80-vector that also codes for MBP-TRC40, generating pQE80-MBP-TRC40/HZZ-emerin-opsin. For pEF-HA-emerin, the emerin coding sequence was amplified from emerin-GFP and cloned into pEF-HA (Gasteier et al., 2003) through SpeI and NcoI. To generate pGEM3Z-Emerin-opsin, a KpnI-HindIII fragment from pET328-HZZ-Emerin-opsin was cloned into pGEM3Z.

Emerin mutants Pro183His, Pro183Thr, Leu225Arg-FS, Trp226*, Gln228His, $\Delta$ Val236-Phe241 and Phe240His-FS were generated in pQE80-MBP-TRC40/HZZ-emerin-opsin and in pEF-HA-emerin by Quikchange site-directed mutagenesis (Braman et al., 1996) or SPRINP methods (Edelheit et al., 2009), using appropriate oligonucleotides (list available upon request).

The FKBP12 sequence for the plasmid coding for $\mathrm{EGFP}_{2}-\mathrm{GST}-\mathrm{NLS}-$ FKBP12 was obtained by PCR amplification from pcDNA3-FKBP12 (Belshaw et al., 1996) and inserted into a pEGFP-C1-derivative coding for $\mathrm{EGFP}_{2}-\mathrm{GST}-\mathrm{NLS}$ (kindly provided by Detlef Doenecke, Göttingen, Germany) through BamHI and XbaI.

Human FRB (the minimal FKBP12-rapamycin-binding domain from FRAP, 2025-2114 fragment; Chen et al., 1995) was PCR amplified from CD4-FRAP-pcDNA3 and cloned into pmCherry-C1 (Clontech) through $\mathrm{BglII}$ and XhoI, generating pmCherry-FRB. The emerin sequence was then introduced through XhoI and BamH1, generating pmCherry-FRB-emerin.

Emerin mutants as above were amplified from pEF-HA-emerin mutant vectors and cloned into pmCherry-FRB through XhoI and BamHI. The point mutation pmCherry-FRB-emerin Gln228His was generated by Quikchange mutagenesis in pmCherry-FRB-emerin-WT. To obtain pcDNA-WRB-FRB-HA, the sequences for human WRB and FRB were PCR-amplified from pRK5-WRB-HA (Vilardi et al., 2011) and pmCherryFRB and cloned through NheI-HindIII or HindIII-BamHI, respectively, into a pcDNA3.1(+) derivative containing an HA tag.

\section{Expression and purification of proteins}

Recombinant MBP-TRC40/HZZ-emerin variant complexes were obtained as described previously (Favaloro et al., 2010) with some modifications. E. coli BL21AI cells were transformed with pQE80-MBP-TRC40/HZZemerin-opsin variants. Expression of MBP-TRC40 was induced at an optical density at $600 \mathrm{~nm}\left(\mathrm{OD}_{600}\right)$ of 0.5 with $50 \mu \mathrm{M}$ IPTG for $1 \mathrm{~h}$ at $30^{\circ} \mathrm{C}$, followed by induction of HZZ-emerin expression with $0.25 \%$ arabinose for $4 \mathrm{~h}$. Cells were harvested and lysed by sonification in cold LS buffer (50 $\mathrm{mM}$ Hepes, $150 \mathrm{mM}$ potassium acetate, $5 \mathrm{mM} \mathrm{Mg}(\mathrm{OAc})_{2}, 10 \%$ glycerol, $1 \mathrm{mM}$ PMSF, $1 \mathrm{mM}$ DTT, pH 7.0) supplemented with $20 \mathrm{mM}$ imidazole and $10 \mu \mathrm{g} / \mathrm{ml}$ DNase I. The lysate was cleared at $100,000 \boldsymbol{g}$ for $30 \mathrm{~min}$ and incubated with a Ni-NTA resin. After $1 \mathrm{~h}$, the resin was sequentially washed with LS buffer supplemented with $5 \mathrm{mM}$ ATP, HS buffer (50 mM Hepes, $500 \mathrm{mM}$ KOAc, $5 \mathrm{mM} \mathrm{Mg}(\mathrm{OAc})_{2}, 10 \%$ glycerol, $1 \mathrm{mM}$ PMSF, $1 \mathrm{mM}$ DTT, pH 7.0) and LS buffer. All washing buffers were supplemented with $20 \mathrm{mM}$ imidazole. The proteins were eluted with LS buffer containing $300 \mathrm{mM}$ imidazole and incubated with an amylose resin for $1 \mathrm{~h}$. The resin was washed with LS buffer supplemented with $5 \mathrm{mM}$ ATP, with HS buffer and again with LS buffer, and the recombinant complex was eluted with LS buffer containing $20 \mathrm{mM}$ maltose.
Expression of the terminal cytosolic domain of CAML (GST-CAML-N) (Yamamoto and Sakisaka, 2012) and of the coiled-coil domain of WRB (MBP-WRBcc) (Vilardi et al., 2011) was as described previously.

Cell culture, transfections and immunofluorescence microscopy

HeLa P4 cells (Charneau et al., 1994), were obtained from the NIH AIDS Reagent Program and were grown in DMEM (Gibco) supplemented with $10 \%(\mathrm{v} / \mathrm{v})$ FCS (Gibco), $100 \mathrm{U} / \mathrm{ml}$ penicillin, $100 \mu \mathrm{g} / \mathrm{ml}$ streptomycin, and $2 \mathrm{mM}$ L-glutamine (Gibco) under $5 \% \mathrm{CO}_{2}$ at $37^{\circ} \mathrm{C}$. They were tested for contamination by mycoplasma on a regular basis. For immunofluorescence, cells were grown on poly-L-lysine-coated coverslips and transfected with the calcium phosphate method (Chen and Okayama, 1987) unless specified otherwise. For siRNA-mediated knockdown, cells were transfected with Lipofectamine (Invitrogen) for $48 \mathrm{~h}$ according to manufacturer's instructions using siRNAs against TRC40 (s1675, Ambion) or a nontargeting control siRNA (AM4635, Ambion).

Cells were fixed with $3.7 \%(\mathrm{v} / \mathrm{v})$ formaldehyde in PBS and permeabilized with $0.5 \%$ Triton X-100 in PBS for 5 min on ice, except for the differential permeabilization experiments. Samples were blocked with either 2\% BSA or $10 \%$ FCS in PBS for 30 min and incubated with primary antibodies diluted in blocking buffer for $1.5 \mathrm{~h}$ at room temperature. Incubation with secondary antibodies was performed for $1 \mathrm{~h}$ in blocking buffer at room temperature. In siRNA experiments, cells were fixed in $100 \%$ methanol at $-20^{\circ} \mathrm{C}$ for $5 \mathrm{~min}$

Cells were mounted with Mowiol-DAPI and analyzed using an Axiovert $200 \mathrm{M}$ fluorescence microscope with a $63 \times$ Plan-Neofluar $1.3 \mathrm{NA}$ watercorrected objective and appropriate filter settings. Images were taken using an LSM 510-META confocal laser scanning microscope (Zeiss) and processed using ImageJ and Adobe Photoshop 6.0.

For quantification of the knockdown effects on the fluorescent signal, a small circle was drawn at the nuclear envelope or in the cytoplasm and fluorescence intensities were determined after background subtraction. Averages were calculated for each treatment group and plotted relative to the highest value (control siRNA), which was set to $100 \%$

\section{Antibodies and western blotting}

For immunodepletions, rat monoclonal anti-HSC70 (1:1000, Stressgen) and rabbit anti-TRC40 (Favaloro et al., 2010) antibodies were used. For indirect immunofluorescence, mouse anti-HA antibodies (1:1000, Convance or Sigma), mouse-anti-TRC40 (1:200, Sigma), rabbit anti-emerin (1:300, Proteintech), rabbit-anti-LBR (1:300, Proteintech), rabbit-anti-STT3B (kind gift from Stephen High, Manchester) and anti-lamin A/C (1:200, Abcam) were used as primary antibodies and donkey-anti-mouse-IgG conjugated to Alexa Fluor 488 or 594 (1:1000; Molecular Probes) as secondary antibodies. Mouse anti-opsin (Adamus et al., 1991), rabbit anti-calnexin (1:1000, Enzo), rabbit-anti-TRC40 (1:1000, Proteintech) and rabbit-anti emerin (1:1000, Santa Cruz Biotechnology) antibodies were used for immunoblotting. For the PLA, mouse anti-myc (1:1000; Santa Cruz Biotechnology), rabbit antiemerin (1:2000, Proteintech), rabbit anti-Sec61 $\beta$ (kind gift from Bernhard Dobberstein, Heidelberg), rabbit anti-STT3B or mouse anti-lamin A/C (1:1000, Abcam) antibodies were used. Western blotting was performed according to standard methods using the Odyssey system (Licor) for secondary antibody detection. For siRNA experiments, chemiluminescence (Millipore) was used. Statistical significance of the data was analyzed by a two-tailed Student's $t$-test. $P<0.05$ was considered as biologically significant.

\section{Microsome integration assay}

Membrane integration of emerin variants was evaluated as previously described (Favaloro et al., 2010; Vilardi et al., 2011). Briefly, two equivalents of rough microsomes (Walter and Blobel, 1983) were incubated for $1 \mathrm{~h}$ at $30^{\circ} \mathrm{C}$ with $200 \mathrm{ng}$ of HZZ-emerin-opsin and MBP-TRC40 complexes in a final volume of $20 \mu \mathrm{l}$ in the absence or presence of $1 \mathrm{mM}$ ATP.

Where indicated, MBP-WRB coiled-coil domain (MBP-WRBcc) or the GST-CAML-N terminal cytosolic domain (GST-CAML-N) were added to a final concentration of $10 \mu \mathrm{M}$. In some experiments, glycosylation or phosphorylation of opsin-tagged emerin was confirmed by treatment of the samples with either 1000 units of EndoH (New England Biolabs), or 400 units of lambda phosphatase (New England Biolabs), in a final volume of 
$40 \mu \mathrm{l}$, according to the instructions of the manufacturer. The efficiency of membrane integration was monitored by SDS-PAGE, followed by western blotting and signal detection using the Odyssey system (Licor). In order to avoid the interference of phosphorylated emerin in the quantification of glycosylation, lambda phosphatase treatment was carried out after the insertion. Signals were quantified using Image Studio Lite (Licor) and expressed as the percentage of N-glycosylated emerin compared with total emerin. Statistical significance of the data was analyzed as above.

\section{Membrane integration in permeabilized cells}

HeLa P4 cells grown on plastic dishes were trypsinized and washed with culture medium and PBS. $4 \times 10^{6}$ cells were resuspended in $400 \mu$ transport buffer [TPB; $20 \mathrm{mM}$ HEPES, $110 \mathrm{mM} \mathrm{KOAc,} 2 \mathrm{mM} \mathrm{Mg}(\mathrm{OAc})_{2}, 1 \mathrm{mM}$ EGTA, pH 7.3, $2 \mathrm{mM}$ DTT, $0.1 \mathrm{mM}$ PMSF and $1 \mu \mathrm{g} / \mathrm{ml}$ each of leupeptin, pepstatin and aprotinin] and permeabilized with a final concentration of $0.01 \%$ digitonin. Cells were washed three times with TPB and resuspended in TPB at 10,000 cells $/ \mu 1$. In a reaction volume of $100 \mu 1,200,000$ cells were incubated with $200 \mathrm{ng}$ of the MBP-TRC40 and HZZ-emerin-WT complex and $1 \mathrm{mM}$ lithium-ATP (Roche) for $1 \mathrm{~h}$ at $30^{\circ} \mathrm{C}$, followed by centrifugation at $16,000 \mathrm{~g}$ for $20 \mathrm{~min}$. After one washing step with TPB, the cells were dissolved in SDS sample buffer and subjected to SDS-PAGE and immunoblotting. For deglycosylation, cells were treated with 500 units PNGaseF (NEB) in a $20 \mu 1$ reaction after membrane integration.

\section{Proximity ligation assay}

HeLa cells were transfected with a plasmid coding for TRC40-myc using Lipofectamine 2000 (Invitrogen). At $48 \mathrm{~h}$ post transfection, cells were fixed and permeabilized as described above. Cells were then subjected to the proximity ligation assay (PLA) using the Duolink red kit (O-link Bioscience) in a humidity chamber according to the manufacturer's instructions. Briefly, cells were blocked, incubated with appropriate primary antibodies and thereafter incubated with PLA probes, which are secondary antibodies (anti-mouse- $\operatorname{IgG}$ and anti-rabbit- $\operatorname{IgG}$ ) conjugated to unique oligonucleotides. Samples were then treated with a ligation solution (allowing oligonucleotide pairs in close proximity to form a closed circle) followed by an amplification solution containing polymerase and fluorescently labeled oligonucleotides, allowing rolling-circle amplification and detection of discrete fluorescent dots. After the PLA protocol, cells were counterstained with anti-Myc monoclonal antibody (Santa Cruz Biotechnology) and Alexa-Fluor-488-conjugated (Molecular Probes) secondary antibody to visualize TRC40-Myc transfected cells. Cells were mounted using Duolink mounting medium with DAPI (O-link Bioscience) and analyzed by confocal laser scanning microscopy with a $63 \times$ water objective. 100 cells, over two independent experiments, were imaged for analysis and each red dot was scored as a single interaction site by eye or using the Duolink Image Tool software.

\section{Differential permeabilization assay}

HeLa cells were transfected with plasmids coding for HA-tagged emerin variants and permeabilized either with $0.007 \%$ digitonin in TPB for $5 \mathrm{~min}$ on ice prior to fixation with $3.7 \%$ formaldehyde in PBS or first fixed and then permeabilized with $0.3 \%$ Triton $\mathrm{X}-100$ and $0.05 \%$ SDS in PBS for $10 \mathrm{~min}$ at room temperature. Cells were then subjected to immunofluorescence microscopy.

\section{Rapamycin assay}

HeLa cells were grown on coverslips and transfected with plasmids coding for $\mathrm{EGFP}_{2}-\mathrm{GST}-\mathrm{NLS}-\mathrm{FKBP} 12$ and an FRB-containing protein of interest. After $24 \mathrm{~h}$, they were permeabilized with $0.007 \%$ digitonin in TPB containing protease inhibitors for $5 \mathrm{~min}$ on ice, washed in TPB and treated with $200 \mathrm{nM}$ rapamycin (Sigma) in TPB for $10 \mathrm{~min}$ on ice. Cells were washed again with PBS, fixed with $3.7 \%$ formaldehyde in PBS and subjected to immunofluorescence microscopy.

\section{Coupled in vitro transcription and translation}

Reactions were performed in the TnT Quick Coupled Transcription/ Translation System (Promega). Briefly, 200 ng of plasmid DNA (pGEM3Z-
Emerin-opsin) was used and the reaction was carried out for $90 \mathrm{~min}$ at $30^{\circ} \mathrm{C}$. $25 \%$ of the reaction was separated by SDS-PAGE for immunodetection. Immunodepletion of TRC40 or HSC70 was performed as described previously (Johnson et al., 2012).

\section{Acknowledgements}

We are grateful to Stephen High (Manchester) and Bernhard Dobberstein (Heidelberg) for reagents and Stephen High for very helpful discussions. We also wish to thank Lena Musiol for critical reading of the manuscript.

\section{Competing interests}

The authors declare no competing or financial interests.

\section{Author contributions}

J.P., J.R.M., C.J., F.V., B.S and R.H.K. designed research; J.P., J.R.M., C.J., K.R., F.V. and R.H.K. performed research; J.P., J.R.M., C.J., K.R., F.V., B.S and R.H.K. analyzed data; J.P., J.R.M., R.H.K. and B.S. wrote the paper.

\section{Funding}

This study was supported by grants of the Deutsche Forschungsgemeinschaft [grant numbers KE 660/12-1 to R.H.K. and SFB1002, TP B01, to B.S.]; and by the People Progamme (Marie Curie Actions) of the European Union's Seventh

Framework Programme (FP7/2007-2013/) under the Research Executive Agency [grant number 607072 to B.S. and J.R.M.]

\section{References}

Abell, B. M., Pool, M. R., Schlenker, O., Sinning, I. and High, S. (2004). Signal recognition particle mediates post-translational targeting in eukaryotes. EMBO J. 23, 2755-2764.

Abell, B. M., Rabu, C., Leznicki, P., Young, J. C. and High, S. (2007). Posttranslational integration of tail-anchored proteins is facilitated by defined molecular chaperones. J. Cell Sci. 120, 1743-1751.

Adamus, G., Zam, Z. S., Arendt, A., Palczewski, K., McDowell, J. H. and Hargrave, P. A. (1991). Anti-rhodopsin monoclonal antibodies of defined specificity: characterization and application. Vision Res. 31, 17-31.

Beilharz, T., Egan, B., Silver, P. A., Hofmann, K. and Lithgow, T. (2003). Bipartite signals mediate subcellular targeting of tail-anchored membrane proteins in Saccharomyces cerevisiae. J. Biol. Chem. 278, 8219-8223.

Belshaw, P. J., Ho, S. N., Crabtree, G. R. and Schreiber, S. L. (1996). Controlling protein association and subcellular localization with a synthetic ligand that induces heterodimerization of proteins. Proc. Natl. Acad. Sci. USA 93, 4604-4607.

Bione, S., Maestrini, E., Rivella, S., Mancini, M., Regis, S., Romeo, G. and Toniolo, D. (1994). Identification of a novel X-linked gene responsible for EmeryDreifuss muscular dystrophy. Nat. Genet. 8, 323-327.

Boni, A., Politi, A. Z., Strnad, P., Xiang, W., Hossain, M. J. and Ellenberg, J. (2015). Live imaging and modeling of inner nuclear membrane targeting reveals its molecular requirements in mammalian cells. J. Cell Biol. 209, 705-720.

Borgese, N., Gazzoni, I., Barberi, M., Colombo, S. and Pedrazzini, E. (2001). Targeting of a tail-anchored protein to endoplasmic reticulum and mitochondrial outer membrane by independent but competing pathways. Mol. Biol. Cell 12 2482-2496.

Braman, J., Papworth, C. and Greener, A. (1996). Site-directed mutagenesis using double-stranded plasmid DNA templates. Methods Mol. Biol. 57, 31-44.

Brambillasca, S., Yabal, M., Makarow, M. and Borgese, N. (2006). Unassisted translocation of large polypeptide domains across phospholipid bilayers. J. Cell Biol. 175, 767-777.

Burns, L. T. and Wente, S. R. (2012). Trafficking to uncharted territory of the nuclear envelope. Curr. Opin. Cell Biol. 24, 341-349.

Cartegni, L., di Barletta, M. R., Barresi, R., Squarzoni, S., Sabatelli, P., Maraldi, N., Mora, M., Di Blasi, C., Cornelio, F., Merlini, L. et al. (1997). Heart-specific localization of emerin: new insights into Emery-Dreifuss muscular dystrophy. Hum. Mol. Genet. 6, 2257-2264.

Charneau, P., Mirambeau, G., Roux, P., Paulous, S., Buc, H. and Clavel, F. (1994). HIV-1 reverse transcription. A termination step at the center of the genome. J. Mol. Biol. 241, 651-662.

Chen, C. and Okayama, H. (1987). High-efficiency transformation of mammalian cells by plasmid DNA. Mol. Cell. Biol. 7, 2745-2752

Chen, J., Zheng, X. F., Brown, E. J. and Schreiber, S. L. (1995). Identification of an 11-KDa FKBP12-rapamycin-binding domain within the 289-kDa FKBP12rapamycin-associated protein and characterization of a critical serine residue. Proc. Natl. Acad. Sci. USA 92, 4947-4951.

Chen, Y.-C., Umanah, G. K. E., Dephoure, N., Andrabi, S. A., Gygi, S. P., Dawson, T. M., Dawson, V. L. and Rutter, J. (2014). Msp1/ATAD1 maintains mitochondrial function by facilitating the degradation of mislocalized tail-anchored proteins. EMBO J. 33, 1548-1564. 
Colombo, S. F., Longhi, R. and Borgese, N. (2009). The role of cytosolic proteins in the insertion of tail-anchored proteins into phospholipid bilayers. J. Cell Sci. 122 2383-2392.

Demmerle, J., Koch, A. J. and Holaska, J. M. (2012). The nuclear envelope protein emerin binds directly to histone deacetylase 3 (HDAC3) and activates HDAC3 activity. J. Biol. Chem. 287, 22080-22088.

Edelheit, O., Hanukoglu, A. and Hanukoglu, I. (2009). Simple and efficient sitedirected mutagenesis using two single-primer reactions in parallel to generate mutants for protein structure-function studies. BMC Biotechnol. 9, 61.

Ellis, J. A., Craxton, M., Yates, J. R. and Kendrick-Jones, J. (1998). Aberrant intracellular targeting and cell cycle-dependent phosphorylation of emerin contribute to the Emery-Dreifuss muscular dystrophy phenotype. J. Cell Sci. 111, 781-792.

Fairley, E. A., Kendrick-Jones, J. and Ellis, J. A. (1999). The Emery-Dreifuss muscular dystrophy phenotype arises from aberrant targeting and binding of emerin at the inner nuclear membrane. J. Cell Sci. 112, 2571-2582.

Favaloro, V., Spasic, M., Schwappach, B. and Dobberstein, B. (2008). Distinct targeting pathways for the membrane insertion of tail-anchored (TA) proteins. J. Cell Sci. 121, 1832-1840.

Favaloro, V., Vilardi, F., Schlecht, R., Mayer, M. P. and Dobberstein, B. (2010) Asna1/TRC40-mediated membrane insertion of tail-anchored proteins. J. Cell Sci. 123, 1522-1530.

Foisner, R. and Gerace, L. (1993). Integral membrane proteins of the nuclear envelope interact with lamins and chromosomes, and binding is modulated by mitotic phosphorylation. Cell 73, 1267-1279.

Furukawa, K., Pante, N., Aebi, U. and Gerace, L. (1995). Cloning of a cDNA for lamina-associated polypeptide 2 (LAP2) and identification of regions that specify targeting to the nuclear envelope. EMBO J. 14, 1626-1636.

Gasteier, J. E., Madrid, R., Krautkrämer, E., Schröder, S., Muranyi, W., Benichou, S. and Fackler, O. T. (2003). Activation of the Rac-binding partner FHOD1 induces actin stress fibers via a ROCK-dependent mechanism. J. Biol. Chem. 278, 38902-38912.

Hegde, R. S. and Keenan, R. J. (2011). Tail-anchored membrane protein insertion into the endoplasmic reticulum. Nat. Rev. Mol. Cell Biol. 12, 787-798.

Hessa, T., Meindl-Beinker, N. M., Bernsel, A., Kim, H., Sato, Y., Lerch-Bader, M. Nilsson, I., White, S. H. and von Heijne, G. (2007). Molecular code fo transmembrane-helix recognition by the Sec61 translocon. Nature 450 1026-1030.

Hradsky, J., Raghuram, V., Reddy, P. P., Navarro, G., Hupe, M., Casado, V., McCormick, P. J., Sharma, Y., Kreutz, M. R. and Mikhaylova, M. (2011). Posttranslational membrane insertion of tail-anchored transmembrane EF-hand Ca2+ sensor calneurons requires the TRC40/Asna1 protein chaperone. J. Biol. Chem 286, 36762-36776.

Johnson, N., Vilardi, F., Lang, S., Leznicki, P., Zimmermann, R. and High, S. (2012). TRC40 can deliver short secretory proteins to the Sec61 translocon. J. Cell Sci. 125, 3612-3620.

Kalbfleisch, T., Cambon, A. and Wattenberg, B. W. (2007). A bioinformatics approach to identifying tail-anchored proteins in the human genome. Traffic 8 1687-1694

King, M. C., Patrick Lusk, C. and Blobel, G. (2006). Karyopherin-mediated import of integral inner nuclear membrane proteins. Nature 442, 1003-1007.

Korfali, N., Wilkie, G. S., Swanson, S. K., Srsen, V., de las Heras, J., Batrakou, D. G., Malik, P., Zuleger, N., Kerr, A. R. W., Florens, L. et al. (2012). The nuclear envelope proteome differs notably between tissues. Nucleus 3, 552-564.

Kralt, A., Jagalur, N. B., van den Boom, V., Lokareddy, R. K., Steen, A. Cingolani, G., Fornerod, M. and Veenhoff, L. M. (2015). Conservation of inner nuclear membrane targeting sequences in mammalian Pom121 and yeast Heh2 membrane proteins. Mol. Biol. Cell 26, 3301-3312.

Kutay, U., Hartmann, E. and Rapoport, T. A. (1993). A class of membrane proteins with a C-terminal anchor. Trends Cell Biol. 3, 72-75.

Laba, J. K., Steen, A. and Veenhoff, L. M. (2014). Traffic to the inner membrane of the nuclear envelope. Curr. Opin. Cell Biol. 28, 36-45

Laba, J. K., Steen, A., Popken, P., Chernova, A., Poolman, B. and Veenhoff, L. M. (2015). Active nuclear import of membrane proteins revisited. Cells 4 653-673

Leach, N., Bjerke, S. L., Christensen, D. K., Bouchard, J. M., Mou, F., Park, R. Baines, J., Haraguchi, T. and Roller, R. J. (2007). Emerin is hyperphosphorylated and redistributed in herpes simplex virus type 1-infected cells in a manner dependent on both UL34 and US3. J. Virol. 81, 10792-10803.

Lee, K. K., Haraguchi, T., Lee, R. S., Koujin, T., Hiraoka, Y. and Wilson, K. L. (2001). Distinct functional domains in emerin bind lamin A and DNA-bridging protein BAF. J. Cell Sci. 114, 4567-4573.

Leznicki, P., Clancy, A., Schwappach, B. and High, S. (2010). Bat3 promotes the membrane integration of tail-anchored proteins. J. Cell Sci. 123, 2170-2178.

Lin, F., Blake, D. L., Callebaut, I., Skerjanc, I. S., Holmer, L., McBurney, M. W., Paulin-Levasseur, M. and Worman, H. J. (2000). MAN1, an inner nuclear membrane protein that shares the LEM domain with lamina-associated polypeptide 2 and emerin. J. Biol. Chem. 275, 4840-4847.

Maimon, T., Elad, N., Dahan, I. and Medalia, O. (2012). The human nuclear pore complex as revealed by cryo-electron tomography. Structure 20, 998-1006.
Manilal, S., Man, N. t. and Morris, G. E. (1998a). Colocalization of emerin and lamins in interphase nuclei and changes during mitosis. Biochem. Biophys. Res. Commun 249, 643-647.

Manilal, S., Recan, D., Sewry, C. A., Hoeltzenbein, M., Llense, S., Leturcq, F., Deburgrave, N., Barbot, J.-C., Man, N., Muntoni, F. et al. (1998b). Mutations in Emery-Dreifuss muscular dystrophy and their effects on emerin protein expression. Hum. Mol. Genet. 7, 855-864.

Mariappan, M., Li, X., Stefanovic, S., Sharma, A., Mateja, A., Keenan, R. J. and Hegde, R. S. (2010). A ribosome-associating factor chaperones tail-anchored membrane proteins. Nature 466, 1120-1124

Mateja, A., Paduch, M., Chang, H.-Y., Szydlowska, A., Kossiakoff, A. A., Hegde, R. S. and Keenan, R. J. (2015). Protein targeting. Structure of the Get3 targeting factor in complex with its membrane protein cargo. Science 347, 1152-1155.

Mora, M., Cartegni, L., Di Blasi, C., Barresi, R., Bione, S., di Barletta, M. R., Morandi, L., Merlini, L., Nigro, V., Politano, L. et al. (1997). X-linked EmeryDreifuss muscular dystrophy can be diagnosed from skin biopsy or blood sample. Ann. Neurol. 42, 249-253.

Nagano, A., Koga, R., Ogawa, M., Kurano, Y., Kawada, J., Okada, R., Hayashi, Y. K., Tsukahara, T. and Arahata, K. (1996). Emerin deficiency at the nuclear membrane in patients with Emery-Dreifuss muscular dystrophy. Nat. Genet. 12 254-259.

Nigro, V., Bruni, P., Ciccodicola, A., Polltano, L., Nigro, G., Piluso, G., Cappa, V., Covone, A. E., Romeo, G. and D'Urso, M. (1995). SSCP detection of nove mutations in patients with Emery-Dreifuss muscular dystrophy: definition of a small C-terminal region required for emerin function. Hum. Mol. Genet. 4 2003-2004.

Ognibene, A., Sabatelli, P., Petrini, S., Squarzoni, S., Riccio, M., Santi, S., Villanova, M., Palmeri, S., Merlini, L. and Maraldi, N. M. (1999). Nuclea changes in a case of X-linked Emery-Dreifuss muscular dystrophy. Muscle Nerve 22, 864-869.

Ohba, T., Schirmer, E. C., Nishimoto, T. and Gerace, L. (2004). Energy- and temperature-dependent transport of integral proteins to the inner nuclea membrane via the nuclear pore. J. Cell Biol. 167, 1051-1062.

Okreglak, V. and Walter, P. (2014). The conserved AAA-ATPase Msp1 confers organelle specificity to tail-anchored proteins. Proc. Natl. Acad. Sci. USA 111 8019-8024.

Östlund, C., Ellenberg, J., Hallberg, E., Lippincott-Schwartz, J. and Worman, H. J. (1999). Intracellular trafficking of emerin, the Emery-Dreifuss muscular dystrophy protein. J. Cell Sci. 112, 1709-1719.

Ozawa, R., Hayashi, Y. K., Ogawa, M., Kurokawa, R., Matsumoto, H., Noguchi, S., Nonaka, I. and Nishino, I. (2006). Emerin-lacking mice show minimal moto and cardiac dysfunctions with nuclear-associated vacuoles. Am. J. Pathol. 168 907-917.

Pedrazzini, E., Villa, A., Longhi, R., Bulbarelli, A. and Borgese, N. (2000) Mechanism of residence of cytochrome $b(5)$, a tail-anchored protein, in the endoplasmic reticulum. J. Cell Biol. 148, 899-914

Rabu, C., Wipf, P., Brodsky, J. L. and High, S. (2008). A precursor-specific role for Hsp40/Hsc70 during tail-anchored protein integration at the endoplasmic reticulum. J. Biol. Chem. 283, 27504-27513.

Roberts, R. C., Sutherland-Smith, A. J., Wheeler, M. A., Norregaard Jensen, O. Emerson, L. J., Spiliotis, II, Tate, C. G., Kendrick-Jones, J. and Ellis, J. A (2006). The Emery-Dreifuss muscular dystrophy associated-protein emerin is phosphorylated on serine 49 by protein kinase A. FEBS J. 273, 4562-4575.

Salpingidou, G., Smertenko, A., Hausmanowa-Petrucewicz, I., Hussey, P. J. and Hutchison, C. J. (2007). A novel role for the nuclear membrane protein emerin in association of the centrosome to the outer nuclear membrane. J. Cell Biol. 178, 897-904

Schirmer, E. C., Florens, L., Guan, T., Yates, J. R., III and Gerace, L. (2003). Nuclear membrane proteins with potential disease links found by subtractive proteomics. Science 301, 1380-1382.

Schuldiner, M., Metz, J., Schmid, V., Denic, V., Rakwalska, M., Schmitt, H. D., Schwappach, B. and Weissman, J. S. (2008). The GET complex mediates insertion of tail-anchored proteins into the ER membrane. Cell 134, 634-645.

Söderberg, O., Gullberg, M., Jarvius, M., Ridderstråle, K., Leuchowius, K.-J., Jarvius, J., Wester, K., Hydbring, P., Bahram, F., Larsson, L.-G. et al. (2006) Direct observation of individual endogenous protein complexes in situ by proximity ligation. Nat. Methods 3, 995-1000

Soullam, B. and Worman, H. J. (1995). Signals and structural features involved in integral membrane protein targeting to the inner nuclear membrane. J. Cell Biol. 130, 15-27.

Stefanovic, S. and Hegde, R. S. (2007). Identification of a targeting factor for posttranslational membrane protein insertion into the ER. Cell 128, 1147-1159.

Tarpey, P. S., Smith, R., Pleasance, E., Whibley, A., Edkins, S., Hardy, C. O'Meara, S., Latimer, C., Dicks, E., Menzies, A. et al. (2009). A systematic, large-scale resequencing screen of X-chromosome coding exons in mental retardation. Nat. Genet. 41, 535-543.

Tsuchiya, Y., Hase, A., Ogawa, M., Yorifuji, H. and Arahata, K. (1999). Distinct regions specify the nuclear membrane targeting of emerin, the responsible protein for Emery-Dreifuss muscular dystrophy. Eur. J. Biochem. 259, 859-865. 
Ungricht, R. and Kutay, U. (2015). Establishment of NE asymmetry-targeting of membrane proteins to the inner nuclear membrane. Curr. Opin. Cell Biol. 34, 135-141.

Ungricht, R., Klann, M., Horvath, P. and Kutay, U. (2015). Diffusion and retention are major determinants of protein targeting to the inner nuclear membrane. J. Cell Biol. 209, 687-704.

Vaughan, A., Alvarez-Reyes, M., Bridger, J. M., Broers, J. L., Ramaekers, F. C., Wehnert, M., Morris, G. E., Whitfield, W. G. F. and Hutchison, C. J. (2001). Both emerin and lamin $\mathrm{C}$ depend on lamin $\mathrm{A}$ for localization at the nuclear envelope. J. Cell Sci. 114, 2577-2590.

Vilardi, F., Lorenz, H. and Dobberstein, B. (2011). WRB is the receptor for TRC40/ Asna1-mediated insertion of tail-anchored proteins into the ER membrane. J. Cell Sci. 124, 1301-1307.

Vilardi, F., Stephan, M., Clancy, A., Janshoff, A. and Schwappach, B. (2014). WRB and CAML are necessary and sufficient to mediate tail-anchored protein targeting to the ER membrane. PLOS ONE 9, e85033.

Vohanka, S., Vytopil, M., Bednarik, J., Lukas, Z., Kadanka, Z., Schildberger, J., Ricotti, R., Bione, S. and Toniolo, D. (2001). A mutation in the X-linked Emery-
Dreifuss muscular dystrophy gene in a patient affected with conduction cardiomyopathy. Neuromuscul. Disord. 11, 411-413.

Walter, P. and Blobel, G. (1983). Preparation of microsomal membranes for cotranslational protein translocation. Methods Enzymol. 96, 84-93.

Wheeler, M. A., Davies, J. D., Zhang, Q., Emerson, L. J., Hunt, J., Shanahan, C. M. and Ellis, J. A. (2007). Distinct functional domains in nesprin-1alpha and nesprin-2beta bind directly to emerin and both interactions are disrupted in X-linked Emery-Dreifuss muscular dystrophy. Exp. Cell Res. 313, 2845-2857.

Yamamoto, Y. and Sakisaka, T. (2012). Molecular machinery for insertion of tailanchored membrane proteins into the endoplasmic reticulum membrane in mammalian cells. Mol. Cell 48, 387-397.

Yates, J. R. W., Bagshaw, J., Aksmanovic, V. M. A., Coomber, E., McMahon, R. Whittaker, J. L., Morrison, P. J., Kendrick-Jones, J. and Ellis, J. A. (1999). Genotype-phenotype analysis in X-linked Emery-Dreifuss muscular dystrophy and identification of a missense mutation associated with a milder phenotype. Neuromuscul. Disord. 9, 159-165.

Zuleger, N., Kerr, A. R. W. and Schirmer, E. C. (2012). Many mechanisms, one entrance: membrane protein translocation into the nucleus. Cell. Mol. Life Sci. 69, 2205-2216. 
Manuscript 2: Mice lacking WRB reveal differential biogenesis requirements of tail-anchored proteins in vivo 


\section{SCIENTIFIC REP}
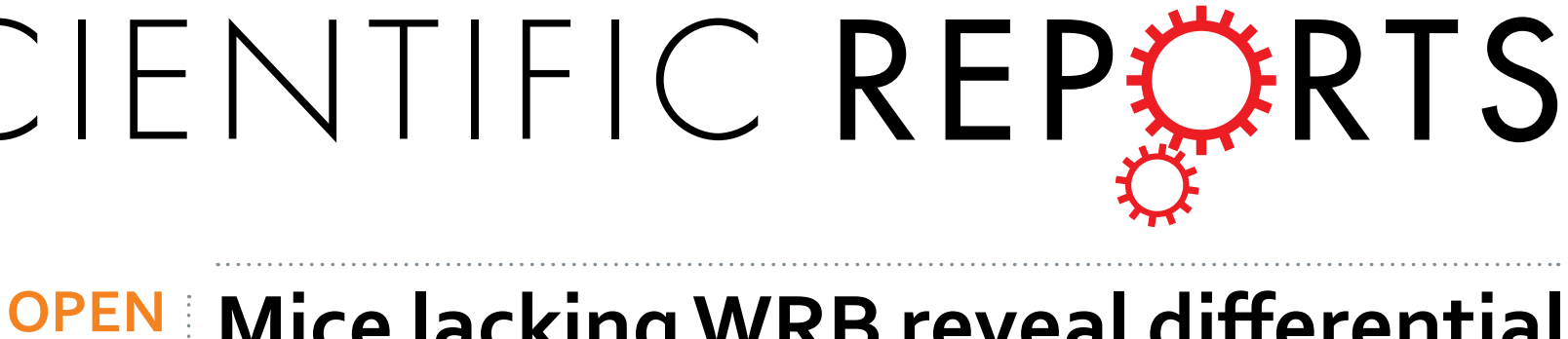

\section{Mice lacking WRB reveal differential biogenesis requirements of tail- anchored proteins in vivo}

Received: 31 August 2016

Accepted: 22 November 2016

Published: 21 December 2016
Jhon Rivera-Monroy ${ }^{1, *}{ }^{*}$, Lena Musiol ${ }^{1, *}{ }^{,}$, Kirsten Unthan-Fechner ${ }^{1}$, Ákos Farkas ${ }^{1}$, Anne Clancy ${ }^{1}$, Javier Coy-Vergara ${ }^{1}$, Uri Weill ${ }^{2}$, Sarah Gockel ${ }^{3}$, Shuh-Yow Lin $^{4,+}{ }^{4}$, David P. Corey ${ }^{4}$, Tobias Kohl ${ }^{3}$, Philipp Ströbel ${ }^{5}$, Maya Schuldiner ${ }^{2}$, Blanche Schwappach ${ }^{1,6}$ \& Fabio Vilardi $^{1}$

Tail-anchored (TA) proteins are post-translationally inserted into membranes. The TRC40 pathway targets TA proteins to the endoplasmic reticulum via a receptor comprised of WRB and CAML. TRC40 pathway clients have been identified using in vitro assays, however, the relevance of the TRC40 pathway in vivo remains unknown. We followed the fate of TA proteins in two tissue-specific WRB knockout mouse models and found that their dependence on the TRC40 pathway in vitro did not predict their reaction to receptor depletion in vivo. The SNARE syntaxin 5 (Stx5) was extremely sensitive to disruption of the TRC40 pathway. Screening yeast TA proteins with mammalian homologues, we show that the particular sensitivity of Stx 5 is conserved, possibly due to aggregation propensity of its cytoplasmic domain. We establish that Stx5 is an autophagy target that is inefficiently membranetargeted by alternative pathways. Our results highlight an intimate relationship between the TRC40 pathway and cellular proteostasis.

The need to target proteins to membranes is a consequence of cellular compartmentalization and thus a fundamental process in all cells. Malleable model organisms such as yeast and reductionist in vitro approaches have enabled the elucidation of the basic mechanisms of membrane targeting. Tail-anchored (TA) membrane proteins were recognized ${ }^{1}$ as a type of precursor protein with specific post-translational targeting requirements due to their topology. They represent a subclass of type-II oriented integral membrane proteins with a single transmembrane domain (TMD) at the extreme C-terminus ${ }^{2}$. In-vitro studies identified the cytosolic ATPase TRC40 (Transmembrane domain Recognition Complex $40 \mathrm{kDa}$ ) as the targeting factor for TA proteins inserted into the membrane of the endoplasmic reticulum (ER) of higher eukaryotes ${ }^{3,4}$. A pre-targeting complex including the proteins BAG6, TRC35, and UBL4A receives newly synthesized TA proteins from the ribosome and transfers them to the cytosolic ATPase TRC40 5 . BAG6 also actively promotes degradation of mislocalized proteins by targeting them to the ubiquitin-proteasome system ${ }^{6,7}$. TRC40 delivers TA proteins to a receptor formed by WRB (Tryptophan Rich Basic protein $)^{8}$ and CAML (Calcium signal-modulating cyclophilin ligand) ${ }^{9}$, two integral membrane proteins localized at the ER.

A conserved pathway in yeast features Get3, the orthologue of TRC40, and a membrane receptor complex comprised of the proteins Get1 and Get 2 as key components ${ }^{10}$. This receptor is thought to work as an insertase for TA proteins at the ER membrane ${ }^{11}$. Many aspects of the molecular mechanism of TRC40/Get3-mediated insertion of TA proteins have been dissected. This dissection gave rise to the current model of TRC40/Get3-dependent targeting that is supported by numerous structural studies of the main components of the pathway in different functional states ${ }^{12-16}$.

${ }^{1}$ Department of Molecular Biology, Universitätsmedizin Göttingen, D-37073 Göttingen, Germany. ${ }^{2}$ Department of Molecular Genetics, Weizmann Institute of Science, Rehovot 7610001, Israel. ${ }^{3}$ Department of Cardiology \& Pulmonology, Universitätsmedizin Göttingen, D-37075 Göttingen, Germany. ${ }^{4}$ Howard Hughes Medical Institute and Department of Neurobiology, Harvard Medical School, Boston, MA, USA. ${ }^{5}$ Institute of Pathology, Universitätsmedizin Göttingen, D-37073 Göttingen, Germany. ${ }^{6}$ Max-Planck Institute for Biophysical Chemistry, D37077, Göttingen, Germany. 'Present address: Department of Surgery, Division of Otolaryngology, UCSD School of Medicine, 9500 Gilman Drive \#0666, La Jolla, CA 92093, USA. *These authors contributed equally to this work. Correspondence and requests for materials should be addressed to B.S. (email: blanche.schwappach@med.unigoettingen.de) or F.V. (email: fabio.vilardi@med.uni-goettingen.de) 
However, the physiological client spectrum of the TRC40 pathway remains to be identified. In fact, a direct role of the pathway was tested for only a few of the several hundreds theoretically predicted TA proteins ${ }^{17}$ based on in vitro membrane integration assays ${ }^{3,4,18}$. Moreover, yeast Get3 is thought to contribute to the targeting of GPI-anchored proteins ${ }^{19}$ and can work as an ATP-independent chaperone under oxidative stress ${ }^{20}$. The latter observation positions the TRC40/Get pathway at the interface of protein biogenesis and quality control. While both processes make use of fundamental mechanisms that require biochemical and biophysical dissection, their physiological bearing and potential contribution to disease development can only be elucidated in the context of differentiated cell types. Insight into the role of the TRC40 pathway in the context of tissue physiology is currently limited.

Although the GET genes are not essential in yeast ${ }^{10}$, loss of the $\mathrm{TRC} 40^{21}$ or CAML-encoding ${ }^{22}$ genes result in early embryonic lethality in a murine model. Generation of conditional knockouts restricted to specific cell types allows the identification of relevant functions of components of the TRC40 pathway in tissue development and physiology. A recent study revealed that pancreatic beta cells lacking TRC40 show impaired insulin secretion ${ }^{23}$, whereas CAML is required for thymocyte development ${ }^{24}$, and loss of either receptor subunit in inner ear cells causes deafness ${ }^{25,26}$.

The locus of the WRB gene (alternatively named CHD5) was mapped to the congenital heart disease region of human chromosome $21^{27}$. A recent study showed increased expression of WRB in Down Syndrome (DS) fetal fibroblasts, in accordance with the presence of an extra copy of the WRB allele ${ }^{28}$. However, a potential correlation between overexpression of WRB and the higher incidence of congenital heart disease in DS patients ${ }^{27}$ remains unclear. Knockdown of WRB in medaka fish leads to defects in cardiac development and ocular abnormalities ${ }^{29}$. Similarly, loss of WRB in Xenopus laevis has a strong impact on cardiac morphology and secretion of basement membrane matrix ${ }^{30}$. Other studies have identified an essential function of WRB in the formation of synaptic structures in photoreceptors and in inner ear cells $s^{26,31,32}$. Due to the physiological focus on the analysis of the phenotypes, i.e. seeing and hearing deficits, and due to the difficulties in analysing inner hair cells and photoreceptor cells biochemically, these studies provide limited information on the general impact of WRB loss on TA protein biogenesis.

Here, we follow the fate of a subset of TA proteins in two terminally differentiated cell types, cardiomyocytes and hepatocytes, lacking WRB. Our results indicate that the TRC40 pathway is not only active in the targeting and membrane insertion of TA proteins but also part of the proteostatic network with effects on the maintenance and quality control of the cellular proteome.

\section{Results}

Experimental Strategy. To investigate the role of the TRC40 pathway in tissue physiology, we employed a previously established mouse line with loxP recombination sites introduced into the WRB allele ${ }^{26}$ allowing tissue-specific knockouts upon crossing with mouse lines expressing the Cre recombinase under the control of a promoter of interest. We created tissue-specific knockout models using Cre lines leading to WRB knockout in adult cardiomyocytes or hepatocytes. These target cells were chosen for the potential relevance of WRB function to their physiology and to disease development. The gene encoding WRB was mapped to human chromosome 21 and is associated with congenital heart disease in Down syndrome patients ${ }^{27}$ and severe cardiac developmental disorders in medaka fish ${ }^{29}$ and Xenopus laevis ${ }^{30}$. Hepatocytes are active secretory cells and therefore require extensive protein targeting.

Knockout of WRB in adult cardiomyocytes results in a TRC40 pathway ko. We crossed the mouse line carrying loxP recombination sites flanking exons 2 and 4 of the WRB gene ${ }^{26}$ with a line expressing a Cre recombinase fused to parts of the estrogen receptor (MerCreMer), under control of the cardiomyocyte-specific myosin 6 (Mhy6) promoter ${ }^{33}$. Tamoxifen injection induces nuclear translocation of the recombinase and therefore allowed WRB depletion in a tissue-specific and temporally controlled manner ${ }^{34}$ (Fig. 1a). Two weeks after tamoxifen administration, we isolated cardiomyocytes using the Langendorff perfusion method ${ }^{35}$. Quantitative PCR showed a strong decrease of WRB mRNA in knockout cells whereas transcript levels of TRC40 and CAML were unchanged (Fig. 1b). Western blot analysis confirmed a strong reduction of WRB protein level but also of CAML and TRC40 (Fig. 1c,d). Interdependence of receptor subunit stability was previously reported for the yeast GET receptor (Get1 and Get2) ${ }^{10}$ as well as for WRB and CAML ${ }^{36}$. A recent study has shown that down-regulation of CAML destabilizes WRB mRNA rather than the protein itself ${ }^{28}$. However, an effect of WRB knockout on CAML mRNA was not observed in our system suggesting that when WRB is missing the major effect is on turnover of the CAML protein.

The analysis of populations of isolated cardiomyocytes revealed residual WRB signals at both the mRNA (Fig. 1b) and the protein level (Fig. 1c). This is unlikely to be due to incomplete recombination ${ }^{34}$ and may well be explained by the presence of contaminating fibroblasts or endothelial cells in the primary cell isolation. As the stability of the WRB protein in isolated adult cardiomyocytes is currently unknown we cannot fully exclude the possibility that some cells retained some WRB protein at the tested time point after knockout induction. Importantly, we observed that the steady-state levels of not only the CAML but also the TRC40 protein were strongly reduced upon cardiomyocyte-specific knockout of WRB.

The steady-state levels of some cardiomyocyte TA proteins are reduced. Next, we analyzed the steady-state levels of a subset of TA proteins by western blot and found that syntaxin 5 (Stx5), an essential SNARE involved in Golgi-to-ER trafficking, and emerin (EMD), a TA protein of the inner nuclear membrane ${ }^{37}$, were significantly less abundant compared to littermates not expressing the recombinase (Fig. 1e,f). In contrast, steady-state protein levels of Sec61 $\beta$, an auxiliary translocon subunit, and syntaxin 6 (Stx6) were not significantly changed and those of syntaxin 8 (Stx8), an endosomal SNARE, not at all affected. Likewise, there was no effect on the steady-state 
a
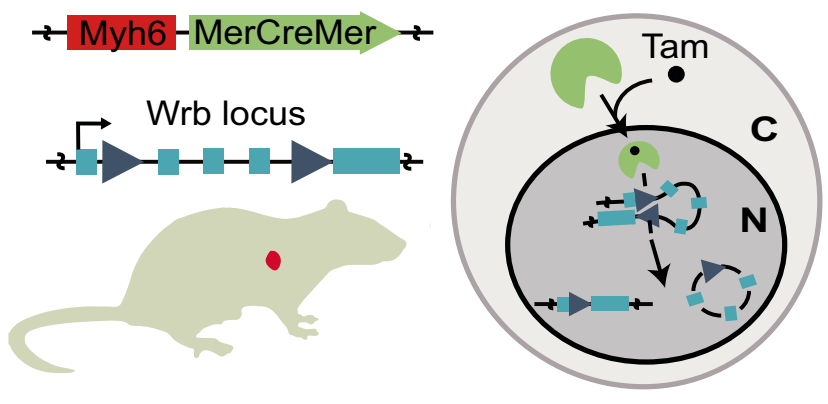

C

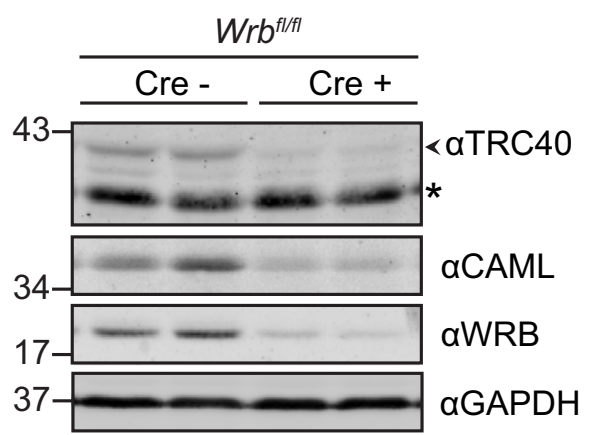

b

Cre-,Wrb ${ }^{f / f f} \square \mathrm{Cre}+, W r b^{f / f l}$

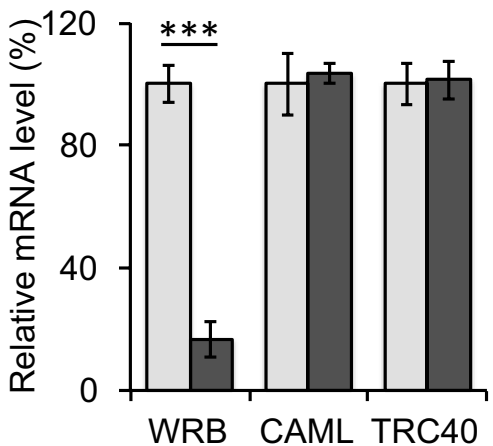

d

$\square$ Cre-,Wrb ${ }^{f / f f l} \square \mathrm{Cre}+, W r b^{f / f f l}$

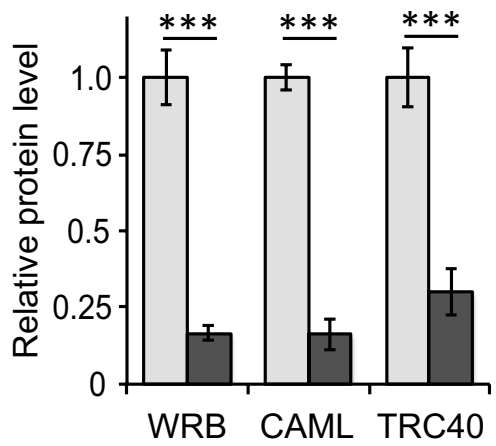

e

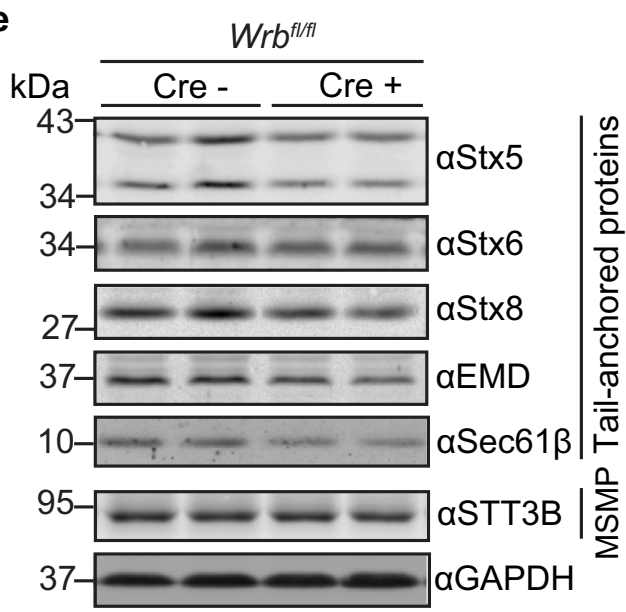

f

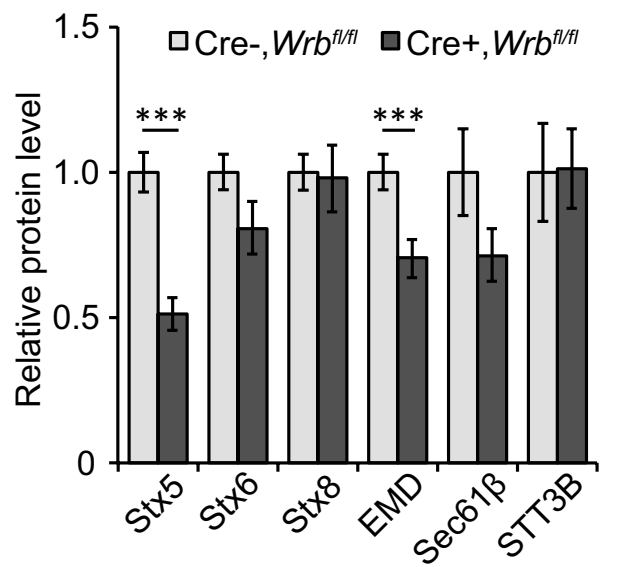

Figure 1. Knockout of WRB in cardiomyocytes results in reduction at steady-state level of TRC40 and CAML as well as Stx5 and emerin (EMD). (a) Schematic of the cardiomyocyte-specific tamoxifen inducible knockout of WRB. Administration of tamoxifen triggers nuclear import of MerCreMer recombinase expressed under the control of Myh6 promoter. This leads to recombination of the WRB locus in the WRB $\mathrm{W}^{\mathrm{f} / \mathrm{fl}}$ mouse line. (b) mRNA levels of the main TRC40-pathway components relative to GAPDH in eight-week old Myh6MerCreMer negative and positive littermates two weeks after tamoxifen induction, measured by qPCR. Bars represent average $+/-$ s.e.m. $\left(\mathrm{n}=4,{ }^{* * *} \mathrm{p}\right.$-value $\left.<0.001\right)$. (c) Cellular lysates from isolated cardiomyocytes were separated by SDS-PAGE and the main TRC40-pathway components were evaluated by western blot using GAPDH as loading control. An asterisk marks a cross-reactive protein. (d) Quantification of the blots as in (c). Bars represent average $+/-$ s.e.m. $\left(n=7-16,{ }^{* * *}\right.$-value $\left.<0.001\right)$. (e) Lysates from isolated cardiomyocytes were separated by SDS-PAGE and expression level of different TA proteins and of the multi spanning membrane protein (MSMP) STT3B was analysed by immunoblot using GAPDH as loading control. (f) Quantification of the blots as in e; bars represent average $+/-$ s.e.m. $\left(n=6-15,{ }^{* * *}\right.$-value $\left.<0.001\right)$.

protein levels of a polytopic control membrane protein, STT3B, a subunit of the protein glycosyltransferase. Quantitative analysis of the transcripts of the tested TA proteins (Supplementary Fig. 1) excluded that the variations observed at the protein level were caused by corresponding changes at the mRNA level. Tamoxifen-injected 
animals of the same age from a control mouse line carrying the MerCreMer recombinase gene and wild type WRB alleles did not show major alterations in the protein levels (Supplementary Fig. 2a-d), excluding side effects of tamoxifen injection, MerCreMer expression, or their combination as the cause of our observations.

CAML and TA protein levels are reduced in WRB ko hepatocytes. To generate a liver-specific WRB knockout, we crossed the same WRB floxed line, with a line expressing Cre recombinase under the control of the constitutively active albumin promoter $^{38}$ (Fig. 2a). In our qPCR analysis, isolated hepatocytes showed a heterogeneous response to recombination. Reduction of WRB mRNA ranged from $5 \%$ to $90 \%$ (Fig. $2 \mathrm{~b}$ ), potentially due to the regeneration capacity of adult hepatocytes ${ }^{39}$ and clonal expansion of cells without the recombined transgene. Independently of the reduction level, all Cre-positive animals showed severe liver damage that was macroscopically visible upon dissection of the animals, and was corroborated by histological sections revealing fibrous areas and by elevated plasma levels of liver enzymes ALT and AST (Supplementary Fig. 4a,b). These strong general effects on the integrity of the liver support previous observations of the essential functions of TRC40 pathway in development and tissue homeostasis ${ }^{21-23,25,29,30}$. In contrast to enzymatic activities indicating liver damage, the serological levels of cholesterol and triglycerides in Cre-positive animals did not change significantly in comparison to control littermates (Supplementary Fig. 4c), indicating sufficient residual liver function.

As in cardiomyocytes, no major differences were observed in the mRNA levels of CAML and TRC40 in hepatocytes (Fig. 2b). We observed that only the few animals that displayed a reduction in WRB mRNA of 70-90\%, showed a significantly reduced protein level of both WRB and CAML, whereas TRC40 levels were not changed significantly (Fig. 2c,d). In addition, these animals confirmed the altered steady-state levels of a subset of TA proteins including Stx5 and Stx6 (Fig. 2e,f) that was not caused by changes at the mRNA level (Supplementary Fig. 1), in accordance with the results obtained in cardiomyocytes (Fig. 1e,f). Animals with a weak reduction of WRB mRNA showed insignificant variations of WRB, CAML and TA protein levels (Supplementary Fig. 4d,e), which may suggest a threshold dependence of the knockout effects.

Sensitive TA proteins mislocalize in cardiomyocytes and hepatocytes. In order to investigate the subcellular localization of client TA proteins as a proxy for successful membrane targeting, we performed indirect immunostaining of isolated cardiomyocytes and hepatocytes. Not all TA proteins studied were severely affected by WRB knockout with respect to their localization. In cardiomyocytes we observed a strong reduction of Stx 5 and Stx6 in vesicular structures resembling the Golgi apparatus (Fig. 3a,b) as confirmed by co-staining with p115 (Supplementary Fig. 3). In about 50\% of the cells, emerin was less clearly localized to the striated cardiomyocyte ER and the nuclear envelope. No effect was observed for the well studied TRC40 substrate Sec61 $\beta^{3,4}$ and Stx 8 (Fig. 3a,b). Evaluation of the localization patterns described above was conducted blindly by three independent investigators using a computational pipeline that shuffles and presents the images without revealing the genotype and an automated decoder that provides the scoring results. Generally, the effects on subcellular localization were correlated with the steady-state protein levels of the tested TA proteins (Fig. 1e,f). TA protein localization was unaltered in Cre-negative or -positive littermate cardiomyocytes from non-floxed animals injected with tamoxifen and analyzed at the same time point after injection (Supplementary Fig. 2e). In knockout hepatocytes, Stx 5 was also an example of a very strongly affected substrate as its Golgi localization was almost completely lost. For Stx8, a TA protein representing the other end of the spectrum based on the protein steady-state levels, we did not observe comparable alterations in targeting to membrane structures although the endomembrane system of Cre-positive hepatocytes appeared slightly perturbed in its overall morphology (Fig. 3c). We conclude that the effect of knocking out the TRC40 receptor leads to a spectrum of biogenesis defects in vivo depending on the TA protein client investigated. For some TA proteins such as the well-established TRC40 client Sec61 $\beta$, the effects were mild whereas some but not all of the syntaxins investigated were severely affected. Indeed, our results highlight Stx 5 and Stx 8 as examples of the respective extremes of TRC40 receptor dependence in vivo and implicate them as useful model proteins for the dissection of the protein features underlying differential TRC40-pathway dependence of TA protein clients.

A systematic yeast screen reveals only 2 strictly GET-dependent clients. In our analysis of endogenous TA proteins in differentiated mammalian cells, the TA proteins that were identified as strictly dependent on the TRC40 pathway were not dedicated to specific cellular functions (such as vesicle fusion) and did not belong to a certain protein family (such as the SNAREs). On the contrary, strong sensitivity of selected clients (e.g. Stx 5 or emerin) may depend on unique features specific to each TA protein. In order to extend our analysis to a greater number of TA proteins we turned to yeast, and performed a systematic screen of yeast TA proteins to determine which substrates are affected in cells lacking the GET pathway ${ }^{10}$.

From a recently generated SWAp-Tag yeast librar ${ }^{40}$, we assembled a subset of yeast strains expressing $\mathrm{N}$-terminally GFP-tagged TA proteins under the control of an intermediate strength, constitutive promoter (NOP1pr). The selection was conducted according to a list of predicted human TA proteins ${ }^{17}$, from which we extracted the 46 with known yeast homologues. We generated deletions of GET pathway genes in all 46 strains, either the double deletion get1/get2 (no GET receptor), single get3 deletion (no cytosolic targeting ATPase), or triple get 1/get2/get3 deletion (no GET pathway) and analyzed them by fluorescence microscopy. This analysis revealed that only two out of the 46 TA proteins were severely affected by deletion of the GET pathway (Fig. 3d and Supplementary Fig. 5a-c). One of the two mislocalized TA proteins was the yeast homolog of Stx5, the well studied SNARE protein Sed5 $5^{10,36,41}$ and the other Lam5, a member of a recently characterized family of lipid transfer proteins ${ }^{42}$. Absolute expression levels, as quantified by flow cytometry measuring the fluorescence intensity of the tested GFP fusion proteins, revealed minor changes for Sed5, Sbh1 and Lam5 in GET mutants and absence of variation for Syn8 (Supplementary Fig. 5b). Western blot assessment of the steady-state levels of endogenous Sed5 implied that the mistargeted protein remained stable (Supplementary Fig. 5d,e), possibly due 
a

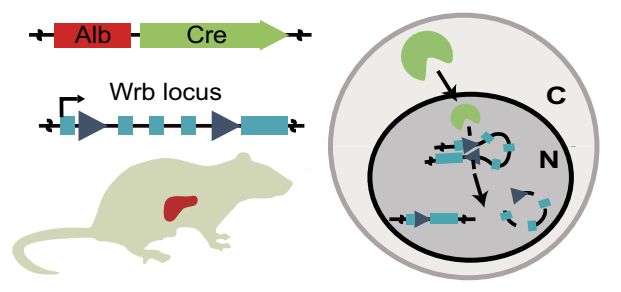

C

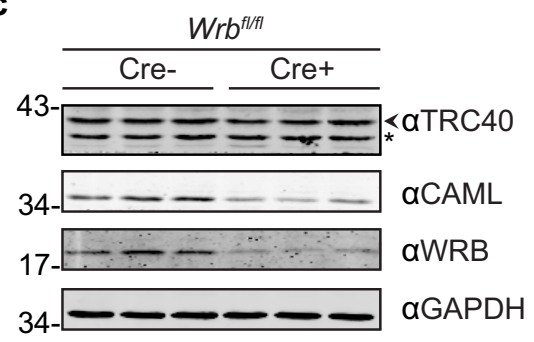

e

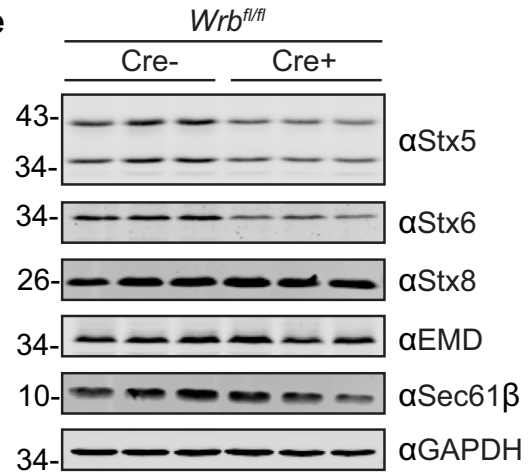

b

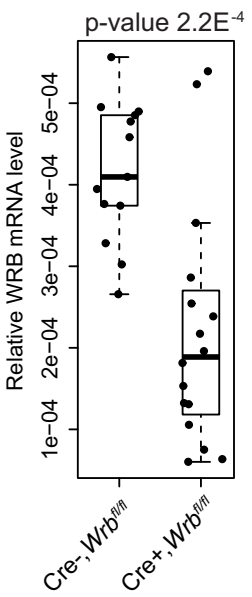

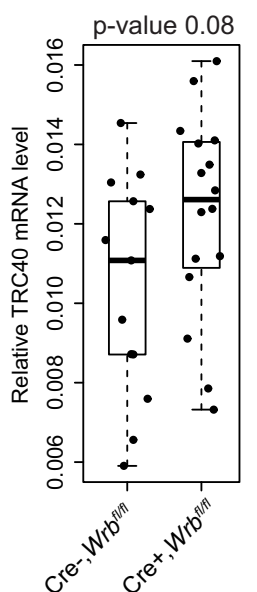

d

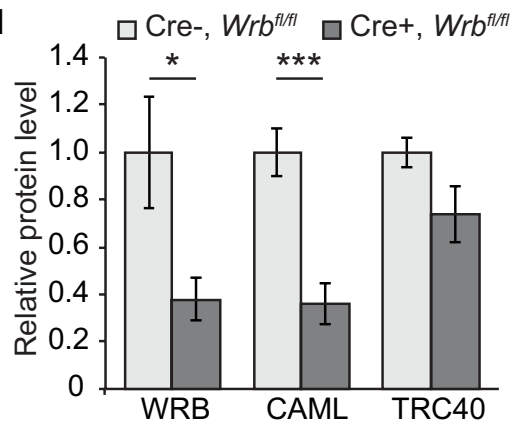

f

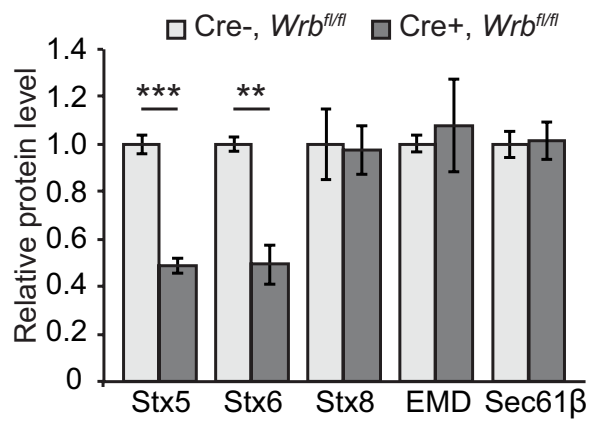

Figure 2. A hepatocyte-specific knockout of WRB leads to a reduction of the TRC40 pathway membrane receptor and substrate TA proteins. (a) Schematic of the hepatocyte specific knockout of WRB. Cre recombinase is expressed under the control of albumin promoter in a $\mathrm{WRB}^{\mathrm{A} / \mathrm{fl}}$ mouse line, allowing recombination of the WRB locus (b) mRNA levels of WRB, CAML and TRC40 were analysed by qPCR. Boxplots depict the levels relative to GAPDH in six-week old Alb-Cre negative and positive littermates. Y-axes show $2^{-\Delta C P}$ values. $p$-values are indicated. (c) Protein lysates from isolated hepatocytes were separated by SDSPAGE and expression level of the main pathway components were evaluated by western blot. Blots of knockout animals in the $25^{\text {th }}$ percentile of the boxplot of WRB mRNA levels in $\mathbf{b}$, and of control animals are shown. GAPDH was used as loading control. An asterisk marks a cross-reactive protein. (d) Quantification of the blots from (c). Bars represent average $-/+$ s.e.m. $\left(n=4\right.$, ${ }^{*}$-value $<0.05$; ${ }^{* * *}$-value $\left.<0.001\right)$. (e) Lysates from isolated hepatocytes were separated by SDS-PAGE and expression level of known substrates of the pathway was evaluated by western blot. Blots of knockout animals in the $25^{\text {th }}$ percentile of the boxplot of WRB mRNA levels in $\mathbf{b}$, and of control animals are shown. GAPDH was used as loading control. (f) Quantifications of the blots from e. Bars represent average $-/+$ s.e.m. $\left(\mathrm{n}=4,{ }^{* *}\right.$ p-value $<0.01{ }^{* * *} \mathrm{p}$-value $\left.<0.001\right)$.

to the action of various chaperones ${ }^{43}$. Importantly, Syn8, the yeast homolog of Stx8, was unaffected (Fig. 3d and Supplementary Fig. 5a,b) as were the 43 other TA proteins screened, including TA proteins that were previously shown to use the GET pathway in vitro such as $\operatorname{Sec} 22^{10}$. Other yeast TA proteins are known to be mislocalized in cells lacking the GET receptor upon over-expression from a strong inducible promoter, such as Sbh1 (homolog of Sec61 $\beta$ ) and Ysy6 (homolog of mammalian Ramp4, also demonstrated to depend on TRC40 for targeting in vitro ${ }^{4}$ ). However, in our strains the tested TA proteins were expressed at levels closer to the endogenous 
a

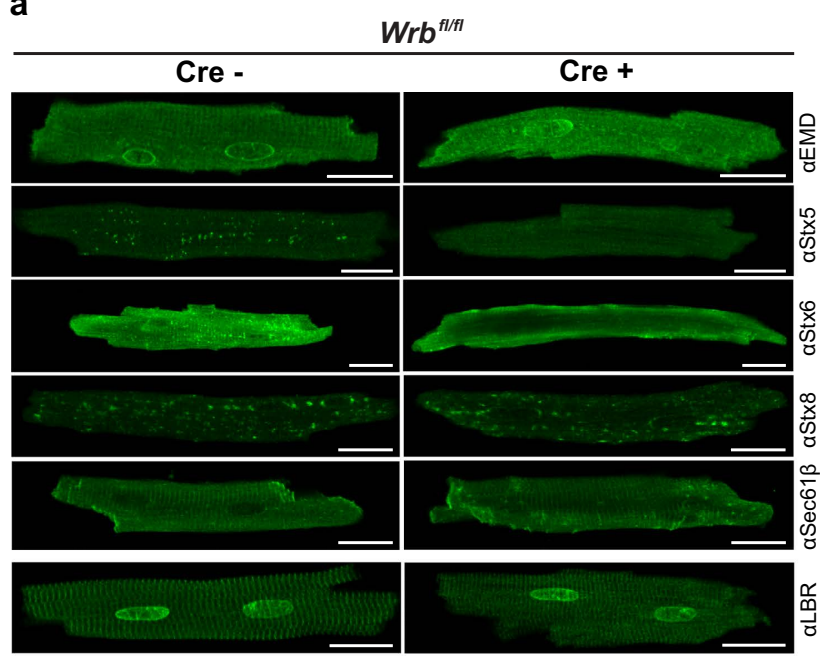

C

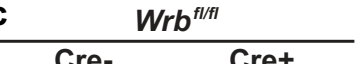

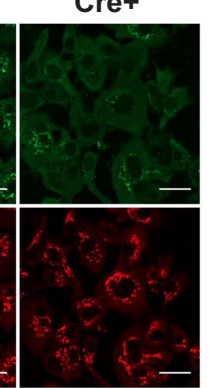
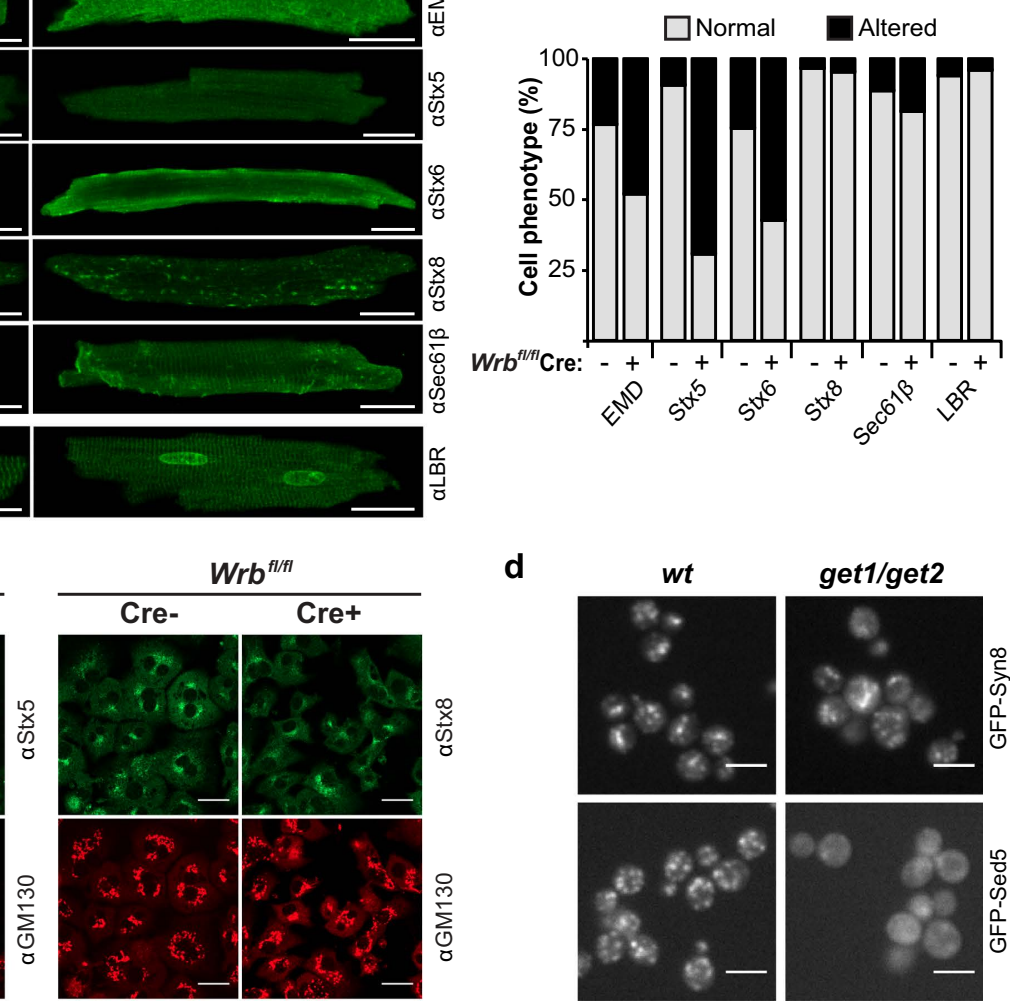

d

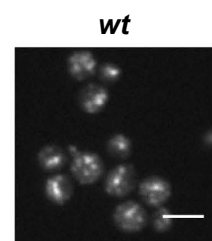

get1/get2
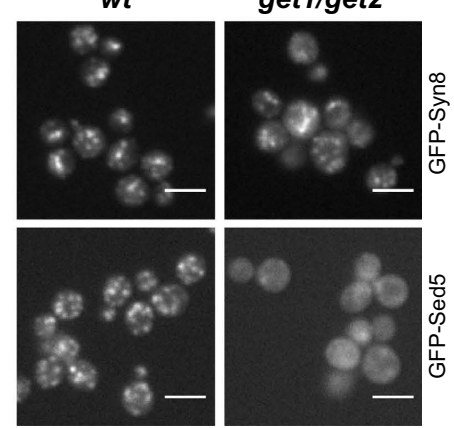

Figure 3. Depletion of the TRC40 pathway receptor results in mislocalization of a subset of TA-proteins in vivo. (a) Ventricular cardiomyocytes from eight-week old MerCreMer- (control) and MerCreMer+ (KO) $\mathrm{Wrb}^{\mathrm{f} / \mathrm{fl}}$ littermates two weeks after tamoxifen induction were isolated and subcellular localization of selected TA proteins (EMD, Stx5, Stx6, Stx8, Sec61 $\beta$ ) was analysed by indirect immunofluorescence. Images were acquired with a confocal microscope. The multi-spanning membrane protein LBR served as control. Scale bar: $20 \mu \mathrm{m}$. (b) Quantification of mislocalization phenotype. For each protein, 22 to 98 cells isolated from 4 to 8 animals were examined. The scoring was performed blindly by three investigators using an image shuffling pipeline and automated genotype/phenotype decoder. The following criteria were applied to assign an "altered" phenotype: for Stx5, Stx6 and Stx8 loss of staining at membraneous structures resembling Golgi or endosomes; for Sec61 $\beta$ loss of staining at cellular striations resembling the sarcoplasmic reticulum; for emerin and LBR loss of staining at the nuclear rim and sarcoplasmic reticulum striations. (c) Isolated hepatocytes from six-week old animals were immunostained for either Stx 5 or Stx 8 and images were acquired with a confocal microscope. GM130 was used as a Golgi marker. Scale bar: $20 \mu \mathrm{m}$. (d) Live-cell microscopy of wild type and get 1/get 2 yeast cells expressing GFP-Sed5 or GFP-Syn8. Scale bar: $5 \mu \mathrm{m}$.

situation and this may explain why they did not show significant variations in the screen. Importantly, the systematic yeast screen confirms a graded dependence of clients on the GET pathway with Stx5 (Sed5) and Stx8 (Syn8) representing the ends of the spectrum.

Stx 5 is not stabilized by proteasomal inhibition. It has been proposed that strongly hydrophobic transmembrane domains strictly require TRC40-mediate targeting whereas more hydrophilic ones can be substrates of alternative pathways ${ }^{44}$. Our results show that Stx 5 is more dependent on the TRC40 pathway despite its transmembrane domain being less hydrophobic than that of Stx8 (47.6 and 61.3 respectively according to the Kyte-Doolittle scale ${ }^{45}$ ). We tested Stx5 and Stx8 in an in-vitro transcription/translation reaction coupled with insertion into canine pancreatic rough microsomes (RM). When the reaction was performed using rabbit reticulocyte lysate depleted of TRC40, membrane integration of both Stx 5 and Stx8 was strongly inhibited (Fig. 4a-c). This result clearly shows that both proteins can be targeted by TRC40 in vitro and reveals that additional molecular mechanisms may govern TA protein biogenesis in vivo.

BAG6 is a component of the TA protein pre-targeting complex but is also known to be involved in the degradation of mislocalized proteins via the ubiquitin-proteasome system ${ }^{6,7}$. Together with our finding that Stx 5 protein levels were lower in mammalian WRB knockout cells (Figs 1e,f and 2e,f) while those of Stx 8 were nearly unchanged, this dual function of the BAG6-containing pre-targeting complex led us to test in HeLa cells whether Stx 5 was targeted for proteasomal degradation in the absence of the TRC40 receptor. Cells were transfected with 
a

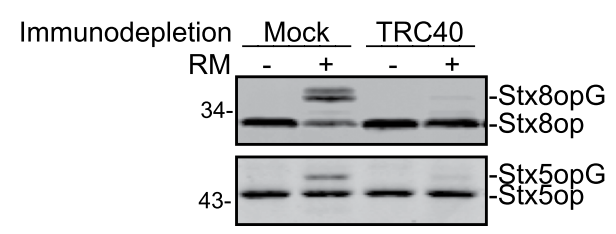

b

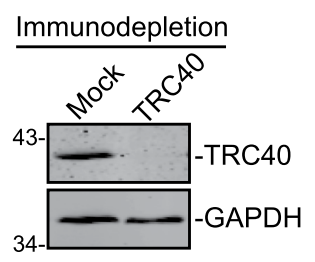

c

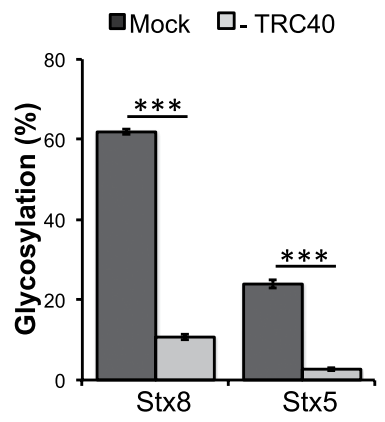

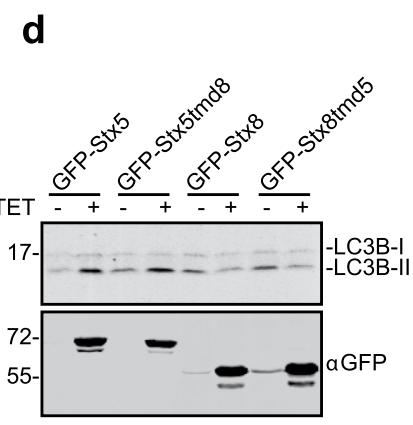

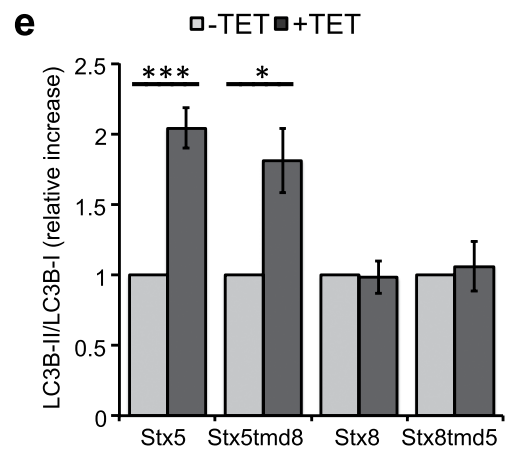

$\mathbf{f}$

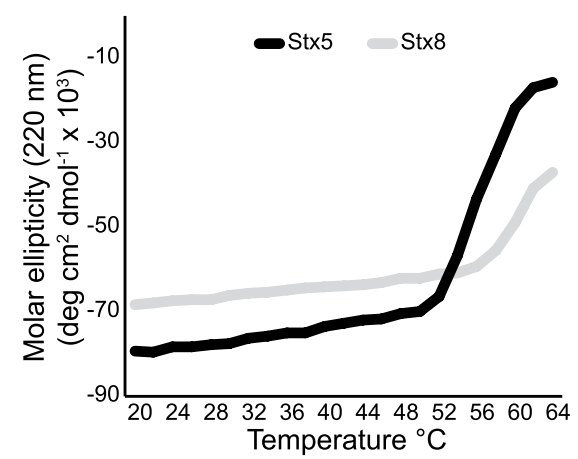

Figure 4. The cytoplasmic domain of Syntaxin 5 is aggregation-prone and can induce autophagy. (a) Stx 5 op and Stx8op were translated in vitro in a rabbit reticulocyte lysate or in a lysate immunodepleted of TRC40. (b) Integration into rough microsomal membranes (RM) was detected by glycosylation of the C-terminal opsin tag (opG) resulting in a slower-migrating protein detected by an opsin-specific antibody. (c) Quantification of glycosylation, percentage of total. Bars represent average $+/-$ s.e.m. $\left(\mathrm{n}=4{ }^{* * *}\right.$ - -value $\left.<0.001\right)$. (d) Stably transfected HEK Flp-In T-REx cells were cultured in absence or presence of tetracycline for 16 hours to induce expression of Stx5/Stx 8 constructs. Cellular lysates were separated by SDS-PAGE and induction was verified by anti-GFP western blot. An increased ratio between the lipidated (LC3B-II) compared to non-lipidated (LC3B-I) LC3B was evaluated by blot and quantified in e Bars represent average $+/$-s.e.m. $\left(\mathrm{n}=4\right.$, ${ }^{*} \mathrm{p}$-value $<0.05{ }^{* * *} \mathrm{p}$-value $\left.<0.001\right)$. (f) Far-UV circular dichroism measured at $220 \mathrm{~nm}$ and increasing temperatures for MBP-Stx 5 cyt (black trace) and MBP-Stx8cyt (gray trace).

siRNA to specifically knock down WRB or TRC40 and were treated with the proteasomal inhibitor oprozomib (OPZ). No significant variations in steady-state levels were observed for Stx 5 or Stx 8 (Supplementary Fig. 6a,b) therefore suggesting that mistargeted Stx 5 is not degraded by the proteasome.

The cytoplasmic domain of Stx 5 is prone to aggregation and induces autophagy. Another cellular process to degrade proteins or even entire organelles is autophagy ${ }^{46}$. We generated HEK293 cells allowing tetracycline-inducible overexpression of Stx5, Stx8, or chimeric proteins with swapped transmembrane domains and monitored autophagy induction via the relative levels of the lipidated form of LC3B, a known autophagosomal marker ${ }^{47}$. Overexpression of Stx 5 or a variant of Stx 5 with the transmembrane domain (TMD) of Stx 8 (Stx5tmd8), but not full-length Stx8 or the inverse chimera, significantly increased the ratio of lipidated versus non-lipidated LC3B, indicating increased levels of autophagosomal membranes (Fig. 4d,e). This result strongly suggests that physicochemical properties of the cytoplasmic domain of Stx 5 are responsible for the observed autophagy induction.

As autophagy is responsible for the clearance of protein aggregates, we wondered if the cytoplasmic domain of Stx 5 has a higher tendency to misfold or aggregate compared to that of Stx8. We purified the cytoplasmic domains of Stx5 (333 amino acids) and Stx8 (216 amino acids) as MBP-tagged recombinant proteins and performed circular dichroism (CD) spectroscopy to monitor changes in secondary structures upon heating. We found that the cytoplasmic domain of Stx 5 had a higher thermal instability and unfolding propensity relative to the same domain in Stx8 (Fig. 4f). An in-vitro aggregation assay confirmed these findings by showing that MBP-Stx 5 cyt incubated at $42^{\circ} \mathrm{C}$ and $55^{\circ} \mathrm{C}$ appeared in the insoluble pellet after centrifugation whereas MBP-Stx 8 cyt remained in the soluble fraction (Supplementary Fig. 6c).

We also detected a strong accumulation of TRC40 and a mild but significant stabilization of Stx 5 in cells treated with chloroquine (CQ), a drug that inhibits lysosomal proteases by preventing acidification of lysosomes ${ }^{48}$. In contrast, variation in Stx8 steady-state levels was not significant (Supplementary Fig. 6d-g). These results indicate that Stx 5 is not only a substrate of lysosome-mediated degradation but can also induce autophagy through distinct properties of its cytoplasmic domain. 

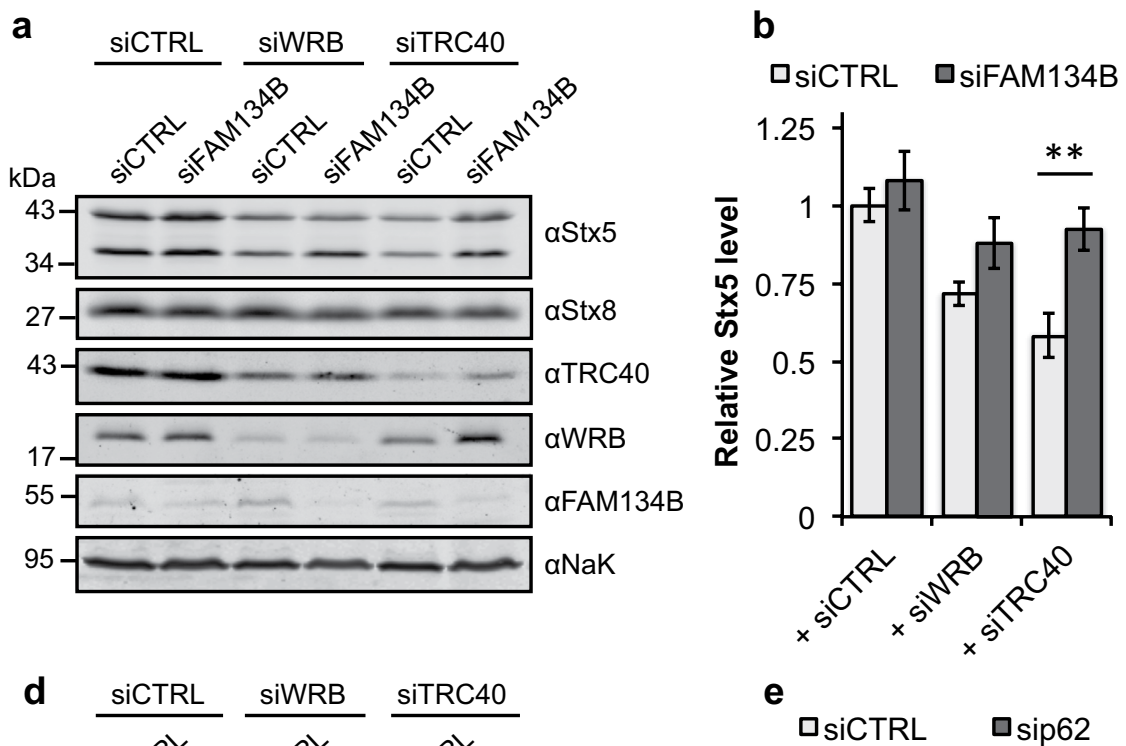

C
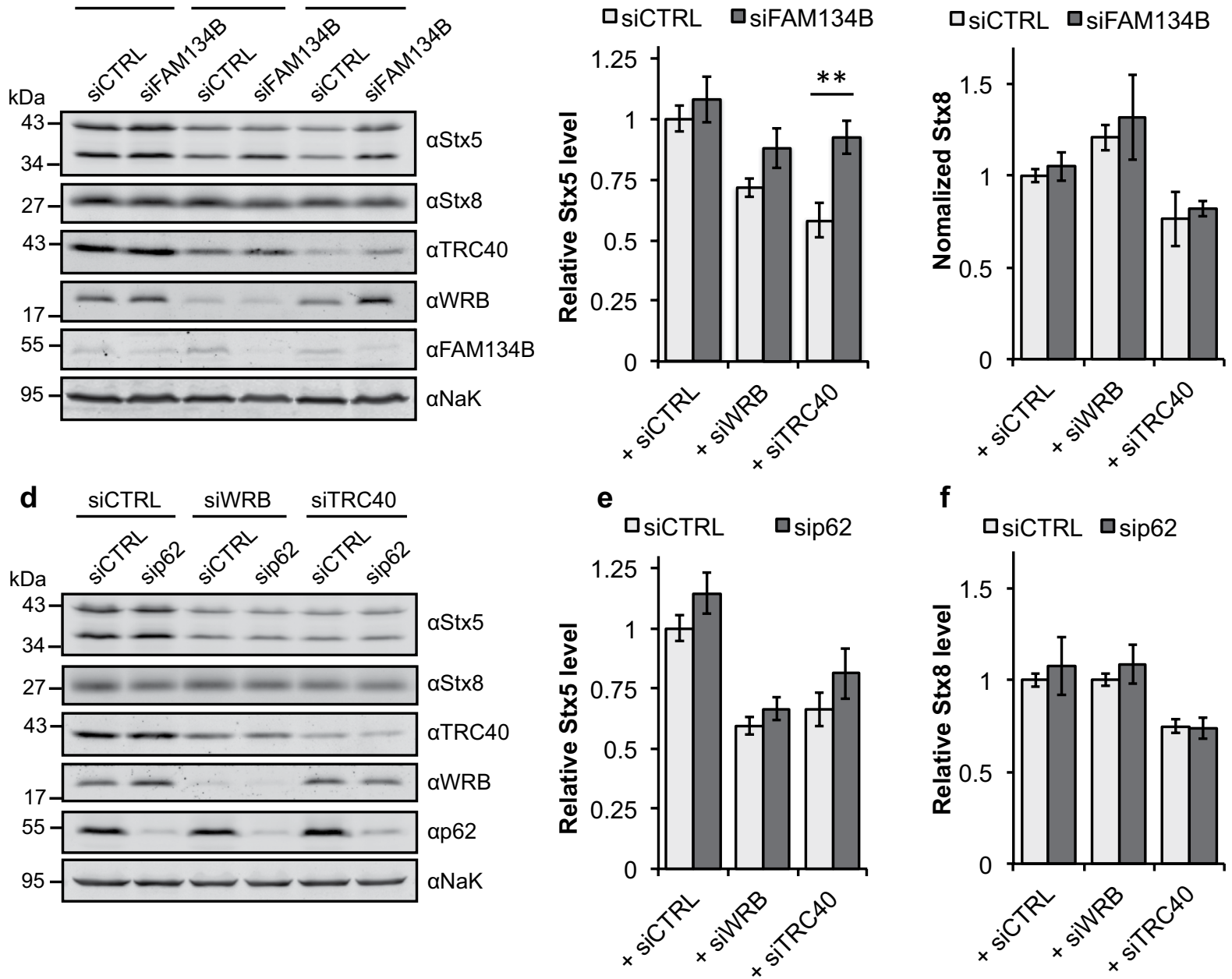

Figure 5. Stx 5 is degraded by a FAM134B-dependent autophagy mechanism. (a) Control-silenced HeLa cells or knockdown cells for WRB or TRC40 were co-transfected with a control siRNA or with a FAM134Bspecific siRNA. Cellular lysates were analyzed by Western blot for the indicated proteins. Graphs in (b,c) show quantification of the expression levels of Stx 5 and Stx8 respectively. Bars represent average $+/-$ s.e.m. $(n=6$, ${ }^{* *}$ p-value $<0.01$ ). (d) Control-silenced HeLa cells or knockdown cells for WRB or TRC40 were co-transfected with a control siRNA or with a p62-specific siRNA. Cellular lysates were analyzed by Western blot for the indicated proteins. Graphs in e,f show quantification of the steady-state levels of Stx 5 and Stx8 respectively. Bars represent average $+/-$ s.e.m. $(\mathrm{n}=4$, variations not significant).

FAM134B is involved in Stx5 degradation. In order to confirm an active role for autophagy in the clearance of Stx5, we used siRNA to selectively down regulate in HeLa cells two autophagy cargo receptors: FAM134B and p62/SQSTM1. FAM134B was recently identified as a member of the reticulon protein family involved in turnover and degradation of the ER and membrane-associated proteins by autophagy ${ }^{49}$. p62/SQSTM1 is a known cargo receptor that mediates autophagosomal degradation of ubiquitinated and non-ubiquitinated cytosolic aggregates $^{50}$.

We observed a significant stabilization of Stx 5 in cells silenced for TRC40 in combination with FAM134B (Fig. 5a,b). Stx8 was not affected by down-regulation of FAM134B (Fig. 5a-c). p62/SQSTM1 silencing stabilized neither Stx 5 nor Stx8 (Fig. 5d-f). These results suggest the existence of a mechanism of Stx 5 degradation after its membrane integration in a FAM134 dependent manner.

\section{Discussion}

The GET/TRC40 pathway was discovered by a combination of experimental strategies using in-vitro biochemical dissection in mammalian lysates, structural biology, and the model organism S. cerevisiae $e^{3,4,10,18}$. These studies identified the key components of the pathway in the cytosol and at the ER membrane. They were also instrumental in demonstrating the interaction of Get3/TRC40 with client TA proteins, with the pre-targeting complex and its ER receptor. These approaches have culminated in a detailed mechanistic model of the targeting process. However, only a small selection of client TA proteins was studied based on practical aspects such as efficient translation in extracts, antibody availability, or ease of recombinant expression and purification. In consequence, the 
actual client spectrum that depends on the pathway in vivo is unknown and so are the properties of clients that make them GET/TRC40 pathway dependent in their targeting.

In higher eukaryotes, TRC40 and the subunits of its receptor WRB and CAML have been investigated based on screens that identified them as being involved in sensory processes ${ }^{25,26,31,32}$. These physiological studies dissect the in-vivo function of the GET/TRC40 pathway proteins from the behavioral phenotype to cellular morphology and detect an impairment of the synaptic endomembrane system that is required for sensory transduction. Interestingly, it was demonstrated that the most critical substrate of WRB-dependent targeting in hair cells of the inner ear is otoferlin. In zebrafish and in mouse, hair-cell specific knockout of WRB lead to hearing deficits that phenocopy loss of otoferlin and can be rescued by overexpression of this TA protein in zebrafish ${ }^{26}$.

We investigated tissue-specific WRB knockout mice to analyze the steady-state levels and subcellular localization of a set of TA proteins including well-established model TA proteins such as Sec61 $\beta$ and different SNAREs in two biochemically amenable differentiated cell types, cardiomyocytes and hepatocytes. We find a graded response of different TA protein clients to loss of the TRC40 receptor with some being much more destabilized and mislocalized than others. In-vitro studies ${ }^{44,51}$ have suggested alternative targeting mechanisms involving cytosolic chaperones such as Hsc70/Hsp40 or SRP and additional SRP-independent targeting pathways for TA proteins may exist ${ }^{19,52}$. Nevertheless, the properties of a substrate that determine its strict dependence on a specific pathway are still unknown.

Together with a recent study showing that Stx 5 and Stx 6 are critically affected TA proteins in pancreatic beta cells lacking TRC $40^{23}$, our results identify Stx 5 as one of the substrates that strictly depends on the TRC40 pathway for its biogenesis. This dependence is conserved in the yeast system and therefore reflects a property of the client protein. We exploited the fact that another SNARE, Stx8, was not affected by loss of WRB in a chimeric approach and found that the cytoplasmic domain of Stx 5 stimulated autophagy in cells overexpressing constructs that included this part of the TA protein irrespective of the transmembrane domain on which it was presented. It has been hypothesized that very hydrophobic transmembrane domains require TRC40 whereas more hydrophilic ones can be substrates of alternative pathways ${ }^{44}$ or capable of spontaneous insertion ${ }^{53}$. Our findings suggest that although properties of the transmembrane domain of TA proteins might be relevant to drive targeting and to ensure the fidelity of membrane integration, the cytoplasmic domain may be even more important for the fate of the protein during biogenesis in vivo.

Accumulation of protein aggregates is a metabolic vulnerability for cells that have to ensure rapid clearance of these aggregates. When membrane targeting via the TRC40 pathway is impaired, due to loss of WRB, the flow of aggregated or misfolded Stx 5 and hence its turnover by autophagy increases. Our results indicate a role of FAM134B in Stx 5 clearance. This protein was recently shown to have an essential function in the turnover of ER by autophagy ${ }^{49}$. Mice lacking FAM134B display an increased ER volume and structure but also a fragmented and elongated Golgi apparatus ${ }^{49}$.

As silencing of FAM134B stabilizes Stx 5 on protein level (Fig. 5a,b) it is conceivable that a certain proportion of Stx 5 is inserted into membrane by alternative targeting mechanisms even in absence of a functional TRC40 pathway. Nevertheless, the observation that Stx 5 is extremely reduced in absence of WRB or TRC40 raises the possibility that Stx 5 targeted to membrane via alternative pathway may be not fully functional. The presence of a non-functional SNARE protein may lead to its active removal and degradation by autophagy. This could be due to many reasons. In the absence of the TRC40 pathway, the targeting fidelity of alternative targeting mechanisms may not support targeting to the right ER domain. It is also conceivable that the TRC40 pathway might mediate the biogenesis of a factor that is essential for the stability and function of Stx5. Another possibility is that Stx 5 is so exquisitely sensitive to loss of the TRC40/GET pathway because it requires the chaperone holdase activity of $\mathrm{TRC} 40 / \mathrm{Get}^{20,43}$ in addition to the TA protein targeting function.

In general, our results indicate that the biogenesis of the vast majority of TA proteins is not acutely affected by deletion of the TRC40/GET pathway in vivo. Indeed, decreasing the number of available receptor sites in vitro ${ }^{18}$ or overexpressing a dominant negative mutant of TRC40 in transfected mammalian cells ${ }^{54}$ slows down membrane integration and increases the amount of stalled TA proteins in the cytosol. Cells may then cope with accumulating TA proteins by promoting their membrane integration via alternative pathways or by ensuring the degradation of clients prone to aggregate or to cause proteotoxic stress (i.e. Stx5). Therefore, it may not be appropriate to reduce the model of TA protein biogenesis to a mere sequence of recognition-targeting-integration events. Instead, it may be necessary to consider the process in the context of the cellular proteostatic network that includes additional mechanisms of quality control and degradation. In this view, the extent to which a TA protein is impaired in its biogenesis by loss of the TRC40/GET pathway will depend on the properties of the whole protein, cytoplasmic domain and transmembrane segment, as well as its abundance in a given cell type. Further tissue specificity will arise from the nature of the pertinent cellular proteostasis network (components and their abundance). The phenotypes observed upon TRC40 pathway knockout in the diverse cell types of multicellular organisms will reflect the most vulnerable and important TA proteins in that cell - as illustrated by the consequences of impairing Stx $5 / 6$ biogenesis in pancreatic beta cells for insulin secretion ${ }^{23}$ or by the defects in otoferlin targeting observed in the WRB inner hair cell knockout ${ }^{26}$. These results expand and refine our knowledge on the TRC40 pathway in eukaryotes and provide a first view on its integration into tissue physiology.

\section{Methods}

Cell lines. HeLa P4 cells were grown in DMEM (Gibco) supplemented with 10\% (v/v) FBS (Gibco). Plasmid and siRNA transfections were performed with BBS-calcium phosphate ${ }^{55}$. For siRNA-mediated knockdowns, cells were transfected twice at 48 hours interval, using RNA oligos specific for WRB (sense AAAUCCAACAGGUAAUUCCAACACC, antisense GGUGUUGGAAUUACCUGUUGGAUUU, Invitrogen), TRC40 (s1675, Ambion), FAM134B (sense AGGUAUCCUGGACUGAUAAUG, antisense CAUUAUCAGUCCAGGAUACCU, Sigma Aldrich), p62/SQSTM1 (sense GCAUUGAAGUUGAUAUCGAU, antisense AUCGAUAUCAACUUCAAUGC, Sigma Aldrich), and non targeting control siRNA (AM4635, Ambion). 
Hek Flp-In T-REx were cotransfected with combinations of the recombinase containing vector pOG44 and the pCDNA5-FRT-TO-N-GFP constructs described in the "Plasmids" section. Recombined clones were selected for resistance to Hygromycin B $(150 \mu \mathrm{g} / \mathrm{ml})$. Expression of Stx 5 , Stx 8 or chimeric proteins was induced by adding $1 \mu \mathrm{g} / \mathrm{ml}$ tetracycline to cell medium for 16 hours.

Mice. All the procedures involving animals were reviewed and approved by the Institutional Animal Care and Use Committee of the University Medical Center Göttingen, in compliance with the humane care and use of laboratory animals.

$\mathrm{WRB}^{\mathrm{f} / \mathrm{fl}}$ line was previously described ${ }^{26}$. Myh6-MerCreMer (B6.FVB(129)-Tg(Myh6-cre/Esr1*)1JmK/J) and Albumin-Cre mice (B6.Cg-Tg(Alb-cre)21Mgn/J) were purchased from the Jackson Laboratory.

MerCreMer dependent recombination was induced in six-week old animals by injection of $40 \mathrm{mg} / \mathrm{kg}$ of tamoxifen (diluted in EtOH-soybean oil) as previously described ${ }^{34}$.

Genotyping. Mice tails were harvested before three weeks of age, and lysed for $14-16$ hours at $56^{\circ} \mathrm{C}$ in DirectPCR-Tail Lysis reagent (Peqlab), plus proteinaseK $(20 \mu \mathrm{g} / \mathrm{mL})$, followed by inactivation at $85^{\circ} \mathrm{C}, 45 \mathrm{~min}$. Supernatant was used as PCR template, with MangoTag (Bioline) and primers for wt and transgene as shown in "Primers" Table. Products were then analysed with standard agarose gel electrophoresis.

Primary cells isolation and culture. At 8 weeks of age and two weeks after tamoxifen induction of the MerCreMer recombinase, ventricular cardiac myocytes were isolated by retrograde perfusion with a modified Langendorff ${ }^{35}$ setup with minor changes. Briefly, hearts were perfused at a rate of $4 \mathrm{ml} / \mathrm{min}$ with $\mathrm{Ca}^{2+}$ free oxygenated perfusion buffer for $4 \mathrm{~min}, 37^{\circ} \mathrm{C}$, followed by perfusion with collagenase type II $(600 \mathrm{U} / \mathrm{ml}$ in perfusion buffer supplemented with $12.5 \mu \mathrm{M} \mathrm{CaCl}_{2}$, Worthington Biochemical Corporation) for $8 \mathrm{~min}$ at $37^{\circ} \mathrm{C}$. Digested ventricles were minced in collagenase-containing buffer and the cardiomyocytes were released by gentle pipetting. Digestion was stopped with $10 \%$ bovine calf serum in perfusion buffer, and the cells were washed twice in the same buffer. The quality of the preparations was verified in a Neubauer chamber, and isolations with less than $60 \%$ rod shape cells were discarded. For indirect immunofluorescence microscopy, isolated cardiomyocytes were plated on laminin (BD Biosciences)-coated glass coverslips.

Primary hepatocytes were isolated from six-week old animals by perfusing livers with collagenase and subjecting the isolated cells to a Percoll gradient. Briefly, the liver was perfused via the Vena cava caudalis, using the Vena portae as outflow with $125 \mathrm{~mL}$ Krebs-Ringer/EGTA, followed by perfusion with Krebs-Ringer substituted with Collagenase/HEPES/CaCl${ }_{2}$ for $4 \mathrm{~min}$. After perfusion, the liver was excised, rinsed in Williams $\mathrm{E}$ (Gibco) and pulled apart with forceps to wash out the cells, followed by a filtering step ( $70 \mu \mathrm{m}$ pore size). The cells were then subjected to a Percoll gradient (GE Healthcare). Hepatocytes were washed once and resuspended in Williams E $(1 \mathrm{~g}$ of cells in $50 \mathrm{~mL})$. The amount of cells was quantified using a counting chamber. For indirect immunofluorescence, cells were plated on collagen-coated glass coverslips.

Blood withdrawal. Animals were anesthetized with isoflurane followed by injection of approx. $400 \mu \mathrm{L}$ Nembutal $(10 \mathrm{mg} / \mathrm{mL})$. Blood was taken from the Vena cava caudalis with a small syringe after opening the body cavity. For obtaining serum for the analysis, the syringe was rinsed with lithium-heparine (Sigma-Aldrich) before drawing blood. Samples were then centrifuged at $500 \mathrm{~g}$ for $30 \mathrm{~min}$ to collect the serum. Blood analysis was carried out by the Department of Clinical Chemistry of the University Medical School Göttingen.

Histology. Livers were embedded in Paraffin and $3 \mu \mathrm{m}$ sections were obtained at a microtome. Sections were stained with hematoxylin-eosin or Goldner staining kit (Engelbrecht).

qPCR. mRNA was isolated from samples using a Roche High Pure RNA isolation kit, following manufacturer's instructions. Equal amounts of total RNA were subjected to cDNA synthesis (SuperScript III First-Strand Synthesis System). qPCRs were performed using a Roche SYBR green Master Mix in a Roche Lightcycler480. mRNA levels were normalized to GAPDH mRNA.

Protein extraction and Western Blot. For mammalian samples, equal amounts of cells were lysed in solubilization buffer (50 mM Tris-HCl pH7.4, 10 mM NaCl, 5 mM EDTA, 2.5 mM EGTA, 1.5\% Triton X-100, 0.75\% Na-deoxycholate, $0.1 \%$ SDS) supplemented with protease inhibitors. Proteins were precipitated by TCA before SDS-PAGE separation and immunoblot analysis.

For yeast protein extraction, cells were recovered by low speed centrifugation and resuspended in $100 \mathrm{mM}$ $\mathrm{NaOH}$ for 10 minutes. Cells were pelleted and lysed in SDS loading buffer.

Primary and secondary antibodies were diluted (as described in the "Antibodies" Table) in blocking buffer (5\% milk in PBS, $0.1 \%$ Tween). Blots were imaged using an Odyssey Sa Infrared imaging system with IRDye LiCOR secondary antibodies. Quantification was performed using the ImageStudio Software (LI-COR).

Indirect immunofluorescence microscopy. For immunofluorescence, cells were fixed as described in the "Antibodies" Table. Samples were blocked with 10\% FCS in PBS for $30 \mathrm{~min}$ and incubated with primary antibodies diluted in blocking buffer at $4{ }^{\circ} \mathrm{C}$ overnight. Incubation with Alexa Fluor secondary antibodies (Thermo Fisher Scientific) was performed for 1 hour at room temperature, and the samples were mounted with Mowiol-DAPI.

Images were taken using a LSM 510-META confocal laser scanning microscope (Zeiss) with a $63 \mathrm{x}$ Plan-Neofluar 1.3 NA water-corrected objective and appropriate filter settings. Images were processed using ImageJ software (https://imagej.nih.gov/ij/). 
Blind annotation KNIME workflow. Using a KNIME workflow, images were copied and given a random name after which they were manually annotated. The results of the annotation were decoded using the same workflow. The workflow was written in KNIME 3.2.1 (https://www.knime.org) and requires the KNIME File Handling Nodes (3.2.2.v201609201941) plugin.

Yeast strains. Yeast strains were selected from the SWAp-Tag library ${ }^{40}$. SGA crosses were carried out as described previously ${ }^{56}$ by crossing the strains with a query strain (MAT $\alpha$ his $3 \Delta 1$ leu $2 \Delta 0$ met $15 \Delta 0$ ura $3 \Delta 0$ can $1 \Delta$ $:: S T E 2$ pr-spHIS5 lyp $1 \Delta:: S T E 3 p r-L E U 2)$ carrying combinations of get $1 \Delta:: \mathrm{KAN}$, get $2 \Delta:: \mathrm{NAT}$, and get $3 \Delta:: \mathrm{BLE}$ to yield get $1 /$ get 2 , get 3 , and get $1 /$ get $2 /$ get 3 strains, as indicated. The query strains with and without GET1/GET2/GET3 replacements were also used for Western blots, as indicated.

Live-cell imaging. Logarithmic phase cultures of indicated yeast strains grown in synthetic dropout media were diluted and transferred into BioConext (United Chemical Technologies) and Concanavalin A (Sigma-Aldrich) coated 384-well glass-bottom plates (Matriplate, Brooks Life Science Systems). Microtiter plates were automatically imaged at $30^{\circ} \mathrm{C}$ on an Imaging Machine 03-dual (Acquifer) widefield high content screening microscope, equipped with a white LED array for bright field imaging, an LED fluorescence excitation light source, an sCMOS $(2048 \times 2048$ pixel $)$ camera, a temperature-controlled incubation chamber, and a stationary plate holder in combination with movable optics. Images were acquired with $470 \mathrm{~nm}$ filter cubes (Ex 469/35, Em 525/39, dichroic 497) using $3 \mathrm{z}$-slices $(\mathrm{dz}=1 \mu \mathrm{m})$ and a 40x CFI Super Plan Fluor ELWD N.A. 0.60 (Nikon), using $500 \mathrm{~ms}$ integration time. The focal plane was detected in the bright field channel using a yeast autofocus algorithm.

Flow cytometry. Yeast strains were cultured in synthetic complete media at $30^{\circ} \mathrm{C}$ overnight, diluted to $\mathrm{OD}_{600} 0.2$, cultured for 4 hours at $30^{\circ} \mathrm{C}$, washed and resuspended in PBS. Fluorescence intensity was measured with a BD FACSCanto flow cytometer (BD biosciences). Data were analyzed with custom R scripts using the Bioconductor package FlowCore ${ }^{57}$. GFP fluorescence intensities were represented as Kernel density plots using the ggplot2 graphing package ${ }^{58}$.

In vitro transcription/translation. Stx5op and Stx8op were synthesized using a TnT Quick Coupled Transcription/Translation System (Promega) according to manufacturer instructions. Briefly, 200 ng of plasmid containing the coding sequence of the protein of interest were incubated with $10 \mu \mathrm{l}$ of reaction mixture. After 45 minutes, rough microsomes were added and the reaction was incubated for 45 more minutes. Immunodepletion of TRC40 was performed as previously described ${ }^{59}$.

Protein purification. pQE80-MBPtevStx5cyt and pQE80-MBPtevStx8cyt were transformed into E. coli $\mathrm{BL} 21 \mathrm{AI}$. Induction of recombinant proteins was performed at $30^{\circ} \mathrm{C}$ by addition of $0.5 \mathrm{mM}$ IPTG for 2 hours. Cells were lysed in PBS with an Avestin-Emulsiflex and lysates cleared by centrifugation at 100,000 g for 45 minutes. The lysates were incubated with an amylose resin (NEB) for one hour at $4{ }^{\circ} \mathrm{C}$. The resin was washed three times with ten volumes of PBS and finally the proteins eluted in PBS supplemented with $20 \mathrm{mM}$ maltose.

Aggregation assay and circular dichroism spectrocopy. $\quad 10 \mu \mathrm{M}$ of MBPtevStx 5 cyt or MBPtevStx 8 cyt were incubated at different temperatures (as shown in Supplementary Fig. 6c) for one hour. Insoluble material was separated by centrifugation at $25,000 \mathrm{~g}$ for 20 minutes. Fractions of soluble and insoluble proteins were analysed by SDS-PAGE and stained by coomassie blue.

Changes in the secondary structure of the cytosolic domains of Stx 5 and Stx 8 were determined by recording far-UV CD spectra at $2{ }^{\circ} \mathrm{C}$ temperature intervals (from $20^{\circ} \mathrm{C}$ to $62^{\circ} \mathrm{C}$ ) using a Chirascan qCD (Applied Photophysics). For these measurements, proteins were dialyzed against CD buffer ( $30 \mathrm{mM}$ Na-phosphate $\mathrm{pH} 7$, $300 \mathrm{mM}$ NaF, $0.1 \mathrm{mM}$ TCEP) and diluted to $10 \mu \mathrm{M}$.

\section{References}

1. Kutay, U., Hartmann, E. \& Rapoport, T. A. A class of membrane proteins with a C-terminal anchor. Trends in Cell Biol. 3, 72-75 (1993).

2. Borgese, N. \& Fasana, E. Targeting pathways of C-tail-anchored proteins. Biochim Biophys Acta 1808, 937-946 (2011).

3. Stefanovic, S. \& Hegde, R. S. Identification of a targeting factor for posttranslational membrane protein insertion into the ER. Cell 128, 1147-1159 (2007).

4. Favaloro, V., Spasic, M., Schwappach, B. \& Dobberstein, B. Distinct targeting pathways for the membrane insertion of tail-anchored (TA) proteins. J. Cell. Sci. 121, 1832-1840 (2008)

5. Mariappan, M. et al. A ribosome-associating factor chaperones tail-anchored membrane proteins. nature 466, 1120-1124 (2010).

6. Hessa, T. et al. Protein targeting and degradation are coupled for elimination of mislocalized proteins. nature 475, 394-397 (2011).

7. Krysztofinska, E. M. et al. Structural and functional insights into the E3 ligase, RNF126. Sci Rep 6, 26433 (2016).

8. Vilardi, F., Lorenz, H. \& Dobberstein, B. WRB is the receptor for TRC40/Asna1-mediated insertion of tail-anchored proteins into the ER membrane. J. Cell. Sci. 124, 1301-1307 (2011).

9. Yamamoto, Y. \& Sakisaka, T. Molecular machinery for insertion of tail-anchored membrane proteins into the endoplasmic reticulum membrane in mammalian cells. Mol. Cell 48, 387-397 (2012).

10. Schuldiner, M. et al. The GET complex mediates insertion of tail-anchored proteins into the ER membrane. Cell 134, 634-645 (2008).

11. Wang, F., Chan, C., Weir, N. R. \& Denic, V. The Get1/2 transmembrane complex is an endoplasmic-reticulum membrane protein insertase. nature 512, 441-444 (2014)

12. Mateja, A. et al. Protein targeting. Structure of the Get3 targeting factor in complex with its membrane protein cargo. Science 347, $1152-1155$ (2015).

13. Stefer, S. et al. Structural basis for tail-anchored membrane protein biogenesis by the Get3-receptor complex. Science 333, 758-762 (2011).

14. Mariappan, M. et al. The mechanism of membrane-associated steps in tail-anchored protein insertion. nature 477, 61-66 (2011). 
15. Chartron, J. W., Suloway, C. J., Zaslaver, M. \& Clemons, W. M. J. Structural characterization of the Get4/Get5 complex and its interaction with Get3. Proc. Natl. Acad. Sci. USA 107, 12127-12132 (2010).

16. Chang, Y. W. et al. Crystal structure of Get4-Get5 complex and its interactions with Sgt2, Get3, and Ydj1. The Journal of Biological Chemistry 285, 9962-9970 (2010).

17. Kalbfleisch, T., Cambon, A. \& Wattenberg, B. W. A bioinformatics approach to identifying tail-anchored proteins in the human genome. Traffic 8, 1687-1694 (2007).

18. Favaloro, V., Vilardi, F., Schlecht, R., Mayer, M. P. \& Dobberstein, B. Asna1/TRC40-mediated membrane insertion of tail-anchored proteins. J. Cell. Sci. 123, 1522-1530 (2010).

19. Ast, T., Cohen, G. \& Schuldiner, M. A network of cytosolic factors targets SRP-independent proteins to the endoplasmic reticulum. Cell 152, 1134-1145 (2013).

20. Voth, W. et al. The protein targeting factor Get3 functions as ATP-independent chaperone under oxidative stress conditions. Mol. Cell 56, 116-127 (2014).

21. Mukhopadhyay, R., Ho, Y. S., Swiatek, P. J., Rosen, B. P. \& Bhattacharjee, H. Targeted disruption of the mouse Asnal gene results in embryonic lethality. FEBS Lett. 580, 3889-3894 (2006).

22. Tran, D. D., Russell, H. R., Sutor, S. L., van Deursen, J. \& Bram, R. J. CAML is required for efficient EGF receptor recycling. Dev. Cell 5, 245-256 (2003).

23. Norlin, S., Parekh, V. S., Naredi, P. \& Edlund, H. Asna1/TRC40 Controls $\beta$-Cell Function and Endoplasmic Reticulum Homeostasis by Ensuring Retrograde Transport. Diabetes 65, 110-119 (2016).

24. Tran, D. D. et al. CAML is a p56Lck-interacting protein that is required for thymocyte development. Immunity 23, 139-152 (2005).

25. Bryda, E. C. et al. Conditional deletion of calcium-modulating cyclophilin ligand causes deafness in mice. Mamm. Genome 23, 270-276 (2012)

26. Vogl, C. et al. Tryptophan-rich basic protein (WRB) mediates insertion of the tail-anchored protein otoferlin and is required for hair cell exocytosis and hearing. The EMBO Journal, doi: 10.15252/embj.201593565 (2016).

27. Egeo, A. et al. Identification and characterization of a new human cDNA from chromosome 21q22.3 encoding a basic nuclear protein. Hum Genet 102, 289-293 (1998).

28. Colombo, S. F. et al. Tail-anchored protein biogenesis in mammals: function and reciprocal interactions of the two subunits of the TRC40 receptor. The Journal of Biological Chemistry, doi: 10.1074/jbc.M115.707752 (2016).

29. Murata, K., Degmetich, S., Kinoshita, M. \& Shimada, E. Expression of the congenital heart disease 5/tryptophan rich basic protein homologue gene during heart development in medaka fish, Oryzias latipes. Dev Growth Differ 51, 95-107 (2009).

30. Sojka, S. et al. Congenital heart disease protein 5 associates with CASZ1 to maintain myocardial tissue integrity. Development 141, 3040-3049 (2014).

31. Daniele, L. L., Emran, F., Lobo, G. P., Gaivin, R. J. \& Perkins, B. D. Mutation of wrb, a Component of the Guided Entry of TailAnchored Protein Pathway, Disrupts Photoreceptor Synapse Structure and Function. Invest. Ophthalmol. Vis. Sci. 57, 2942-2954 (2016).

32. Lin, S.-Y. et al. The zebrafish pinball wizard gene encodes WRB, a tail-anchored-protein receptor essential for inner-ear hair cells and retinal photoreceptors. J. Physiol. (Lond.) 594, 895-914 (2016).

33. Sohal, D. S. et al. Temporally regulated and tissue-specific gene manipulations in the adult and embryonic heart using a tamoxifeninducible Cre protein. Circ. Res. 89, 20-25 (2001).

34. Lexow, J., Poggioli, T., Sarathchandra, P., Santini, M. P. \& Rosenthal, N. Cardiac fibrosis in mice expressing an inducible myocardialspecific Cre driver. Dis Model Mech 6, 1470-1476 (2013).

35. Bell, R. M., Mocanu, M. M. \& Yellon, D. M. Retrograde heart perfusion: the Langendorff technique of isolated heart perfusion. J. Mol. Cell. Cardiol. 50, 940-950 (2011).

36. Vilardi, F., Stephan, M., Clancy, A., Janshoff, A. \& Schwappach, B. WRB and CAML are necessary and sufficient to mediate tailanchored protein targeting to the ER membrane. PLoS ONE 9, e85033 (2014).

37. Pfaff, J. et al. Emery-Dreifuss muscular dystrophy mutations impair TRC40-mediated targeting of emerin to the inner nuclear membrane. J. Cell. Sci. 129, 502-516 (2016).

38. Postic, C. et al. Dual roles for glucokinase in glucose homeostasis as determined by liver and pancreatic beta cell-specific gene knock-outs using Cre recombinase. J. Biol. Chem. 274, 305-315 (1999).

39. Taub, R. Liver regeneration: from myth to mechanism. Nat. Rev. Mol. Cell Biol. 5, 836-847 (2004).

40. Yofe, I. et al. One library to make them all: streamlining the creation of yeast libraries via a SWAp-Tag strategy. Nat. Methods 13, 371-378 (2016).

41. Jonikas, M. C. et al. Comprehensive characterization of genes required for protein folding in the endoplasmic reticulum. Science 323, 1693-1697 (2009).

42. Gatta, A. T. et al. A new family of StART domain proteins at membrane contact sites has a role in ER-PM sterol transport. Elife 4, (2015).

43. Powis, K. et al. Get3 is a holdase chaperone and moves to deposition sites for aggregated proteins when membrane targeting is blocked. J. Cell. Sci. 126, 473-483 (2013).

44. Rabu, C., Wipf, P., Brodsky, J. L. \& High, S. A Precursor-specific Role for Hsp40/Hsc70 during Tail-anchored Protein Integration at the Endoplasmic Reticulum. The Journal of Biological Chemistry 283, 27504-27513 (2008).

45. Kyte, J. \& Doolittle, R. F. A simple method for displaying the hydropathic character of a protein. J. Mol. Biol. 157, 105-132 (1982).

46. Kaur, J. \& Debnath, J. Autophagy at the crossroads of catabolism and anabolism. Nat. Rev. Mol. Cell Biol. 16, 461-472 (2015).

47. Tanida, I., Ueno, T. \& Kominami, E. LC3 and Autophagy. Methods Mol. Biol. 445, 77-88 (2008).

48. Mizushima, N., Yoshimori, T. \& Levine, B. Methods in mammalian autophagy research. Cell 140, 313-326 (2010).

49. Khaminets, A. et al. Regulation of endoplasmic reticulum turnover by selective autophagy. nature 522, 354-358 (2015).

50. Katsuragi, Y., Ichimura, Y. \& Komatsu, M. p62/SQSTM1 functions as a signaling hub and an autophagy adaptor. FEBS J. 282, $4672-4678$ (2015).

51. Abell, B. M., Rabu, C., Leznicki, P., Young, J. C. \& High, S. Post-translational integration of tail-anchored proteins is facilitated by defined molecular chaperones. J. Cell. Sci (2007).

52. Aviram, N. et al. The SND proteins constitute an alternative targeting route to the endoplasmic reticulum. Nature, doi: 10.1038/ nature20169.

53. Brambillasca, S. et al. Transmembrane topogenesis of a tail-anchored protein is modulated by membrane lipid composition. The EMBO Journal 24, 2533-2542 (2005).

54. Claessen, J. H. L., Mueller, B., Spooner, E., Pivorunas, V. L. \& Ploegh, H. L. The transmembrane segment of a tail-anchored protein determines its degradative fate through dislocation from the endoplasmic reticulum. The Journal of Biological Chemistry 285, 20732-20739 (2010).

55. Chen, C. \& Okayama, H. High-efficiency transformation of mammalian cells by plasmid DNA. Mol. Cell. Biol. 7, 2745-2752 (1987).

56. Tong, A. H. Y. \& Boone, C. Synthetic genetic array analysis in Saccharomyces cerevisiae. Methods Mol. Biol. 313, 171-192 (2006).

57. Hahne, F. et al. flowCore: a Bioconductor package for high throughput flow cytometry. BMC Bioinformatics 10, 106 (2009).

58. Wickham, H. ggplot2. (Springer, 2016).

59. Leznicki, P., Clancy, A., Schwappach, B. \& High, S. Bat3 promotes the membrane integration of tail-anchored proteins. J. Cell. Sci. 123, 2170-2178 (2010). 


\section{Acknowledgements}

We thank E. Arakel, R. Kehlenbach, King Faisal Yambire, and Nuno Raimundo for extensive discussion and help throughout the study and for valuable comments on the manuscript, T. Moser for generously granting access to the floxed WRB mouse line and for discussion, S. Lehnart for discussion, S. Weber for advice on mouse breeding and liver biology, A. Wolf for technical support of the yeast screen, the UMG animal Facility for excellent mouse husbandry, R. Jahn for access to the CD spectrophotometer and discussion, and N. Lemus-Diaz for comments on the manuscript. The work was supported by SFB1002, TPB01/A07 to B.S. and F.V. and TPA06/A09 to T. Kohl, the Jacob-Henle-Programm to S.G., the ITN TAMPting to J.R.M., J.C.V. and B.S. (funded by the People Progamme (Marie Curie Actions) of the European Union's Seventh Framework Programme (FP7/2007-2013/) under the Research Executive Agency [grant number 607072]), and SFB1190, TP04 to A.C., A.F., and B.S. and TP11 to U.W., M.S., D.P.C. is a Howard Hughes Medical Institute Investigator. M.S. is an Incumbent of the Dr. Gilbert Omenn and Martha Darling Professorial Chair in Molecular Genetics.

\section{Author Contributions}

J.R.M., L.M., A.F., A.C., B.S., and F.V. designed the research, J.R.M., L.M., K.U.-F., A.F., A.C., S.G., T.K., P.S. and F.V. performed experiments, J.R.M., L.M., A.F., A.C., J.C.V., S.G., T.K., P.S., M.S., B.S., and F.V. analyzed data, K.U.-F. maintained the mouse colony including genotyping, S.Y.L. and D.P.C. provided the $\mathrm{WRB}^{\mathrm{f} / \mathrm{fl}}$ mouse line, U.W. and M.S. provided the SWAp-Tag yeast library, J.R.M., L.M., A.F., A.C., B.S., and F.V. wrote the manuscript and all authors commented on the text.

\section{Additional Information \\ Supplementary information accompanies this paper at http://www.nature.com/srep}

Competing financial interests: The authors declare no competing financial interests.

How to cite this article: Rivera-Monroy, J. et al. Mice lacking WRB reveal differential biogenesis requirements of tail-anchored proteins in vivo. Sci. Rep. 6, 39464; doi: 10.1038/srep39464 (2016).

Publisher's note: Springer Nature remains neutral with regard to jurisdictional claims in published maps and institutional affiliations.

(c) T) This work is licensed under a Creative Commons Attribution 4.0 International License. The images
or other third party material in this article are included in the article's Creative Commons license, unless indicated otherwise in the credit line; if the material is not included under the Creative Commons license, users will need to obtain permission from the license holder to reproduce the material. To view a copy of this license, visit http://creativecommons.org/licenses/by/4.0/

(C) The Author(s) 2016 


\section{Supplementary Information}

Mice lacking WRB reveal differential biogenesis requirements of tailanchored proteins in vivo

Jhon Rivera-Monroy ${ }^{+}$, Lena Musiol ${ }^{+}$, Kirsten Unthan-Fechner, Ákos Farkas, Anne Clancy, Javier Coy-Vergara, Uri Weill, Sarah Gockel, Shuh-Yow Lin, David P Corey, Tobias Kohl, Philipp Ströbel, Maya Schuldiner, Blanche Schwappach ${ }^{*}$ and Fabio Vilardi

${ }^{+}$These authors contributed equally to this work.

* Correspondence should be addressed to blanche.schwappach@med.uni-goettingen.de and fabio.vilardi@med.uni-goettingen.de 


\section{Materials}

\section{Plasmids}

For pGem3Z-Stx5op, the coding sequence of Stx5 was amplified from heart cDNA using primers \#1 and \#2. The Kpnl/HindIII fragment was then cloned into pGEM3Z (Promega). pGem3Z-Stx8op was obtained similarly using primers \#3 and \#4

For pQE80-MBP-Stx5cyt, a Kpnl/Xhol fragment was obtained using pGem3ZStx5op as template and primers \#1 and \#5. The fragment was cloned into pQE80-MBPtev ${ }^{1}$. pQE80-MBP-Stx8cyt was obtained similarly using pGem3ZStx8op as template and primers \#3 and \#6.

pCDNA5-FRT-TO-GFPStx5 was obtained by amplification of pGem3Z-Stx5op template with primers \#1 and \#11. The Kpnl/Xhol digested fragment was cloned into pCDNA5-FRT-TO-N-GFP (Invitrogen). pCDNA5-FRT-TOGFPStx8 was obtained similarly using pGem3Z-Stx8op as template and primers \#2 and \#11.

The chimeric Stx5-Stx8 constructs were obtained by fusion PCR.

For pCDNA5-FRT-TO-GFPStx5tmd8 the coding sequence of the cytosolic domain of Stx5 was amplified from pGem3Z-Stx5op using primers \#1 and \#7 whereas the region encoding the transmembrane segment of Stx8 was amplified from pGem3Z-Stx8op using primers \#8 and \#11. The PCR products were mixed in equimolar ratio and used as template for the fusion PCR using primers \#1 and \#11. A Kpnl/Xhol fragment obtained from this final PCR was cloned into pCDNA5-FRT-TO-N-GFP. 
For pCDNA5-FRT-TO-GFPStx8tmd5 the coding sequence of the cytosolic domain of Stx8 was amplified from pGem3Z-Stx8op using primers \#3 and \#9 whereas the region encoding the transmembrane segment of Stx8 was amplified from pGem3Z-Stx5op using primers \#10 and \#11. The PCR products were mixed in equimolar ratio and used as template for the fusion PCR using primers \#1 and \#11. A Kpnl/Xhol fragment obtained from this final PCR was cloned into pCDNA5-FRT-TO-N-GFP.

\section{Supplementary Reference}

1. Favaloro, V., Vilardi, F., Schlecht, R., Mayer, M. P. \& Dobberstein, B. Asna1/TRC40-mediated membrane insertion of tail-anchored proteins. J. Cell. Sci. 123, 1522-1530 (2010). 
Supplementary Table 1: Primers

\begin{tabular}{|c|c|c|c|}
\hline \# & Name & Sequence (5'-3') & Application \\
\hline 1 & Kpnl-Stx5-F & ATACTAGGTACCATGATCCCGCGGAAACGC & cloning \\
\hline 2 & Stx5op-HindIII-R & $\begin{array}{l}\text { TGATATAAGCTTTCAGCCCGTCTTGTTGGAGAAAGGCACGTAGAAGTTT } \\
\text { GGGCCGGCAAGGAAGACCAC }\end{array}$ & cloning \\
\hline 3 & Kpnl-Stx8-F & TATACTGGTACCATGGCCCCAGACCCCTGG & cloning \\
\hline 4 & Stx8op-HindIII-R & $\begin{array}{l}\text { TGATATAAGCTTTCAGCCCGTCTTGTTGGAGAAAGGCACGTAGAAGTTT } \\
\text { GGGCCGTTGGTTGGCCACACTGC }\end{array}$ & cloning \\
\hline 5 & Stx5-Nterm-Xhol-R & ATACTACTCGAGTCACCGATTGGAGGTAAC & cloning \\
\hline 6 & Stx8-Nterm-Xhol-R & ATACTACTCGAGTCACCCACAGGAAGCTGA & cloning \\
\hline 7 & Stx5-Stx8-R & GCAATAAGATCACCATTATCATCCGATTGGAGGTAACTGACTGG & cloning \\
\hline 8 & Stx5-Stx8-F & CCAGTCAGTTACCTCCAATCGGATGATAATGGTGATCTTATTGC & cloning \\
\hline 9 & Stx8-Stx5-R & GGAAGATTTTGACCATGAGCCACCCACAGGAAGCTGACTTTCTG & cloning \\
\hline 10 & Stx8-Stx5-F & CAGAAAGTCAGCTTCCTGTGGGTGGCTCATGGTCAAAATCTTCC & cloning \\
\hline 11 & opsinSTOP-Xhol-R & TATACACTCGAGTCAGCCCGTCTTGTTGGAGAAAGG & cloning \\
\hline 12 & mWRB-Ex1-F & AGCTTCGTGTTCGGGTGTAAC & qPCR \\
\hline 13 & mWRB-Ex2-R & TTGACGGTGGACAGCTCCTG & qPCR \\
\hline 14 & mWRB-Ex3/4-F & CTACATACTACAAGCAGCGCTGATG & qPCR \\
\hline 15 & mWRB-Ex5-R & ATCCAACAGGTGATTCCAATTCCAC & qPCR \\
\hline 16 & mTRC40-F & GTCCTTGTACGAGACGGAGC & qPCR \\
\hline 17 & mTRC40-R & TTGCAGGGTTTCTCAGGGTC & qPCR \\
\hline 18 & mCAML-F & CTCACCACGGCCTAGAACAG & qPCR \\
\hline 19 & mCAML-R & AACTCTTCCGCGGTACTTCC & qPCR \\
\hline 20 & mGAPDH-F & CCAATGTGTCCGTCGTGGATCT & qPCR \\
\hline 21 & mGAPDH-R & GTTGAAGTCGCAGGAGACAACC & qPCR \\
\hline 22 & $\mathrm{mStx5-F}$ & GCGGAAACGCTACGGATCTA & qPCR \\
\hline 23 & $\mathrm{mStx5-R}$ & GAGTGCTGTCACTGCTGCTA & qPCR \\
\hline 24 & mStx6-F & TCTCACGAGTTGGAGAGCAC & qPCR \\
\hline 25 & mStx6-R & AGAGGATGGCTATGGCACAC & qPCR \\
\hline 26 & mStx8-F & GGAGGGGGATCGAAGACAGA & qPCR \\
\hline 27 & $\mathrm{mStx8}-\mathrm{R}$ & GCTTCTTCGCTCATCAGGCT & qPCR \\
\hline 28 & mEMD-F & GACCACCAAGACATACGGGG & qPCR \\
\hline 29 & mEMD-R & AGTGTGCGATGCTCTGGTAG & qPCR \\
\hline 30 & $\mathrm{mSec} 61 \beta-\mathrm{F}$ & ATGTGGCGATTCTACACGGAA & qPCR \\
\hline 31 & $\mathrm{mSec} 61 \beta-\mathrm{R}$ & GCAGCATAAATACAGCAGCGA & qPCR \\
\hline 32 & mSTT3B-F & CTCCAGTCCAAGTGTGGTCC & qPCR \\
\hline 33 & mSTT3B-R & TGATAGCCGTAGTCCCACCA & qPCR \\
\hline 34 & wt-Forward & TTGGTGAGGCTTGTCTCTGGCGATTT & Genotyping \\
\hline 35 & wt-Reverse & GTTGATCTTCCTTTCCAGCCTGGCGTAT & Genotyping \\
\hline 36 & dneo-Forward & AGTGTACACGCGTACTAGTCTAGCGAA & Genotyping \\
\hline 37 & dneo-Reverse & ACTCAAAGGCAACAGCCAAGATAAGCA & Genotyping \\
\hline 38 & olMR3798 & AGGTGGACCTGATCATGGAG & Genotyping \\
\hline 39 & olMR7338 & CTAGGCCACAGAATTGAAAGATCT & Genotyping \\
\hline 40 & olMR7339 & GTAGGTGGAAATTCTAGCATCATCC & Genotyping \\
\hline 41 & olMR8346 & ATACCGGAGATCATGCAAGC & Genotyping \\
\hline 42 & cre-only-F & ACTGACCGTACACCAAAATTTGC & Genotyping \\
\hline 43 & cre-only-R & CCGCATAACCAGTGAAACAGC & Genotyping \\
\hline 44 & Alb-pr2-F & GTATATTAGAGCGAGTCTTTC & Genotyping \\
\hline 45 & cre2_R & ACCTCATCACTCGTTGCATC & Genotyping \\
\hline
\end{tabular}


Supplementary Table 2: Antibodies

\begin{tabular}{|c|c|c|c|c|c|c|c|c|}
\hline \multirow{2}{*}{ Antigen } & \multirow{2}{*}{ Species } & \multirow{2}{*}{ Source } & \multirow{2}{*}{ Cat. No. } & \multirow{2}{*}{ Lot No. } & \multirow{2}{*}{ Dil. (WB) } & \multicolumn{3}{|c|}{ Immunofluorescence dilution, Fixation } \\
\hline & & & & & & Cardiomyocytes & Hepatocytes & HeLa \\
\hline $\begin{array}{l}\mathrm{Na}+/ \mathrm{K}+\mathrm{ATPase} \\
\text { alpha1 subunit }\end{array}$ & Mouse & $\begin{array}{l}\text { Santa Cruz } \\
\text { Biotehnology, inc. }\end{array}$ & SC-21712 & G3004 & 1:1000 & & & \\
\hline GAPDH & Mouse & Biotrend Chemikalien & $5 G 4$ & $\begin{array}{l}\text { 13/06- } \\
\text { G4-C5 }\end{array}$ & $1: 120000$ & & & \\
\hline WRB & Rabbit & Synaptic systems & 324002 & & $1: 500$ & & & \\
\hline $\begin{array}{l}\text { CAML (Mouse } \\
\text { cells) }\end{array}$ & $\begin{array}{l}\text { Guinea } \\
\text { pig }\end{array}$ & Synaptic systems & 359004 & & $1: 500$ & & & \\
\hline TRC40 (Asna1) & Rabbit & Proteintech & 15450-1-AP & & 1:1000 & & & \\
\hline Stx5 & Rabbit & Synaptic systems & 110053 & $\begin{array}{l}110053 / \\
13\end{array}$ & $1: 2000$ & 1:200, PFA & $\begin{array}{l}\text { 1:100, } \\
\mathrm{MeOH}\end{array}$ & $\begin{array}{l}\text { 1:300, } \\
\text { FA-Suc }\end{array}$ \\
\hline Stx6 & Rabbit & Synaptic systems & 110062 & & $1: 1000$ & 1:200, PFA & & \\
\hline Stx8 & Rabbit & Synaptic systems & 110083 & $\begin{array}{l}110083 / \\
9\end{array}$ & 1:1000 & 1:200, PFA & $\begin{array}{l}\text { 1:300, } \\
\mathrm{MeOH}\end{array}$ & $\begin{array}{l}\text { 1:300, } \\
\text { FA-Suc }\end{array}$ \\
\hline Emerin & Rabbit & $\begin{array}{l}\text { Santa Cruz } \\
\text { Biotehnology, inc. }\end{array}$ & sc-15378 & D2412 & 1:1000 & $1: 150, \mathrm{MeOH}$ & & \\
\hline Sec61 $\beta$ & Rabbit & $\begin{array}{l}\text { Bernhard Dobberstein, } \\
\text { Heidelberg }\end{array}$ & & & $1: 500$ & $1: 300, \mathrm{MeOH}$ & & \\
\hline LBR & Rabbit & Proteintech & 12398-1-AP & & $1: 1000$ & 1:500, PFA & & \\
\hline STT3B & Rabbit & $\begin{array}{l}\text { Stephen High, } \\
\text { Manchester }\end{array}$ & & & 1:1000 & & & \\
\hline GM130 & Mouse & $\begin{array}{l}\text { BD Transduction } \\
\text { Laboratories }\end{array}$ & 610823 & 24277 & & & $\begin{array}{l}\text { 1:200, } \\
\mathrm{MeOH}\end{array}$ & $\begin{array}{l}\text { 1:300, } \\
\text { FA-Suc }\end{array}$ \\
\hline p115 & Mouse & $\begin{array}{l}\text { BD Transduction } \\
\text { Laboratories }\end{array}$ & 612260 & & & 1:400, PFA & & \\
\hline Opsin & Mouse & $\begin{array}{l}\text { Bernhard Dobberstein, } \\
\text { Heidelberg }\end{array}$ & & & $1: 1000$ & & & \\
\hline $\begin{array}{l}\text { Poly-ubiquitin } \\
\text { conjugate }\end{array}$ & Mouse & ENZO & $\begin{array}{l}\text { BML- } \\
\text { PW8810 }\end{array}$ & 5021240 & 1:1000 & & & \\
\hline LC3B & Rabbit & Cell Signaling & 3868 & 0009 & $1: 1000$ & & & \\
\hline GFP & Mouse & $\begin{array}{l}\text { Santa Cruz } \\
\text { Biotehnology, inc. }\end{array}$ & sc-9996 & F0713 & $1: 1000$ & & & \\
\hline FAM134B & Rabbit & Ivan Dikic, Frankfurt & & & $1: 1000$ & & & \\
\hline p62/SQSTM1 & Mouse & abcam & ab56416 & $\begin{array}{l}\text { GR2826 } \\
02-1\end{array}$ & $1: 5000$ & & & \\
\hline Sed5 & Rabbit & $\begin{array}{l}\text { Hans Dieter Schmidt, } \\
\text { Göttingen }\end{array}$ & & & $1: 5000$ & & & \\
\hline Vma2 & Mouse & Molecular probes & A-6427 & & $1: 1000$ & & & \\
\hline
\end{tabular}




\section{Supplementary Figure Legends}

Supplementary Figure 1 Quantitative analysis of TA protein mRNAs. mRNA levels of investigated TRC40-pathway substrates relative to GAPDH cardiomyocytes and hepatocytes were determined by qPCR. Bars represent average +/- s.e.m. Variations are not significant by the student's $t$ test.

Supplementary Figure 2 Control experiments addressing putative effects of tamoxifen-induced MerCreMer translocation to the nucleus in a WRB nonfloxed (Wrb ${ }^{\text {wt/wt }}$ ) Cre+ control mouse line. (a) TRC40-pathway components of ventricular cardiomyocytes isolated from 8-week old MerCreMer-positive, tamoxifen-induced control animals were analyzed by Western blot using GAPDH as loading control. An asterisk marks a cross-reactive protein. (b) Quantification of blots as in a. Bars represent average +/- s.e.m. ( $n=5$, * $p$ value $<0.05$ ). (c) TA proteins of ventricular cardiomyocytes isolated from the MerCreMer-positive control animals were analysed by western blot using GAPDH as loading control. (d) Quantification of blots as in c; bars represent average +/- s.e.m. $(n=5)$. (e) Cardiomyocytes were subjected to indirect immunofluorescence staining of TA proteins and confocal images were acquired. LBR, a multi-spanning membrane protein, served as a negative control. Scale bar: $20 \mu \mathrm{m}$.

Supplementary Figure 3 Colocalization of SNARE proteins with a Golgi marker. Cardiomyocytes isolated from WRB f//fl, Cre- and WRB ${ }^{\mathrm{fl} / f 1}$, Cre+ littermates were subjected to indirect immunofluorescence for Stx5, Stx6 and Stx8 in combination with the Golgi marker protein p115. Images were acquired using a confocal microscope. Scale bar: $20 \mu \mathrm{m}$.

Supplementary Figure 4 Characterization of the consequences of WRB knockout in the liver. (a) Paraffin sections of liver were subjected to Goldner or HE staining. Representative images of a WRB ${ }^{\mathrm{fl} / \mathrm{fl}}$, Cre- and a WRB ${ }^{\mathrm{fl} / \mathrm{fl}}$, Cre+ 8-week old animal are shown. Original magnifications: images with Goldner staining 400x, images with HE staining 200x. Goldner staining was used to 
reveal connective tissue. Nuclei appear in dark purple, cytosol in pink, and collagen in light blue. Hematoxylin and Eosin (HE) staining was used to assess general morphology. Nuclei are stained in dark purple, cytosol is stained in pink, and erythrocytes appear red. (b) Blood values of the liver enzymes Aspartate Transaminase (AST) and Alanine Transaminase (ALT) were measured in $\mathrm{WRB}^{\mathrm{fl} / \mathrm{fl}} \mathrm{Cre}$ - and Cre+ six-week old littermates. Graphs show average -/+ s.e.m. ( $n=9$ (Cre-), $n=7 \quad(\text { Cre }+)^{* * *}$-value<0.001). (c) Cholesterol and triglyceride concentration in plasma of WRB ${ }^{f / f l} \mathrm{Cre}-$ and Cre+ six-week old littermates. Graphs show average $-/+$ s.e.m. $(n=4$, the variations are not significant by student's $t$ test). (d) Western blots assessing the correlation between residual WRB mRNA levels and the steady-state protein levels of different TRC40 pathway components in hepatocytes isolated from six-week old animals. Blots of Cre+ littermates of the $25^{\text {th }}(++)$ and $50^{\text {th }}(+)$ percentile of the boxplot in Fig. 2b, together with two Cre- animals are shown. Protein amounts were quantified relative to GAPDH and shown as a bar diagram for each individual blot. An asterisk marks a cross-reactive protein. (e) Western blots assessing the correlation between residual WRB mRNA levels and the steady-state protein levels of the TA proteins Stx5 and Stx8 in hepatocytes isolated from six-week old animals. The same samples as in c were used and analysis was performed as described for $\mathrm{c}$.

Supplementary Figure $\mathbf{5}$ Steady-state protein levels and localization of TA proteins in get mutants lacking either the GET receptor, Get3, or both. (a) Live-cell microscopy images showing localization of selected genomically $\mathrm{N}$ terminally GFP-tagged TA proteins in $S$. cerevisiae get1/get2, get3, and get1/get2/get3 mutant strains. (b) Yeast strains depicted in panel (a) were analyzed by flow cytometry and fluorescence intensity distribution in wild type and get mutant strains are shown. BY4741 strain (gray trace) not expressing GFP was used as a negative control. (c) List of $S$. cerevisiae homologs of predicted human TA proteins indicating the localization of their genomically $\mathrm{N}$ terminally GFP-tagged versions in wt and get1/get2, get3, and get1/get2/get3 yeast strains based on live-cell microscopy. (d) Protein levels of native Sed5 were tested by western blot in wt, get1/get2, get3, and get1/get2/get3 yeast 
strains. The protein Vma2 was used as a loading control. (e) Quantification of blots from c. Bars represent average $-/+$ s.e.m. $\left(n=3,{ }^{* *} p-v a l u e<0.01\right)$.

Supplementary Figure 6 Comparison of Stx5 and Stx8 reveals different requirements for their cellular degradation. (a) Control siRNA treated HeLa cells or siRNA-mediated knockdown cells for WRB or TRC40 were incubated for 4 hours with $10 \mu \mathrm{M}$ oprozomib (OPZ), an inhibitor of the proteasome, or DMSO as control. Cellular lysates were analyzed by Western blot for the indicated proteins. Graph in (b) shows quantification of the steady-state levels of Stx5 and Stx8 relative to the Na/K-ATPase. Bars represent average +/s.e.m. $(n=3)$ (c) Recombinant MBP-Stx5cyt and MBP-Stx8cyt were incubated at the indicated temperature. Soluble and insoluble proteins were separated by centrifugation. Fractions were loaded onto an SDS-PAGE gel and visualized by Coomassie staining. (d) Control silenced HeLa cells or knockdown cells for WRB or TRC40 were treated for 6 hours in the presence or absence of $100 \mu \mathrm{M}$ chloroquine (CQ). Cellular lysates were analyzed by western blot for the indicated proteins. Graphs in (e-g) show quantification of the steady-state levels of TRC40, Stx5, and Stx8. Bars represent average +/s.e.m. $\left(n=6,{ }^{*} p\right.$-value $<0.05,{ }^{* *} p$-value $\left.<0.01\right)$. 


\section{Supplementary Figure 1}
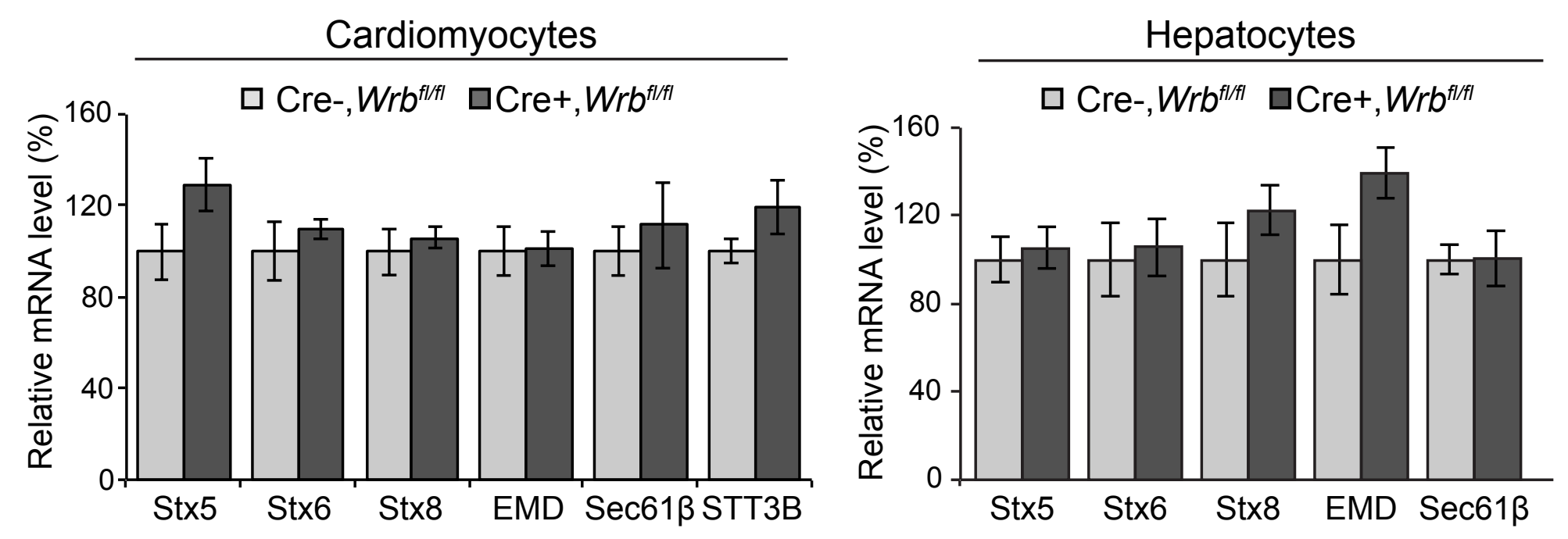


\section{Supplementary Figure 2}

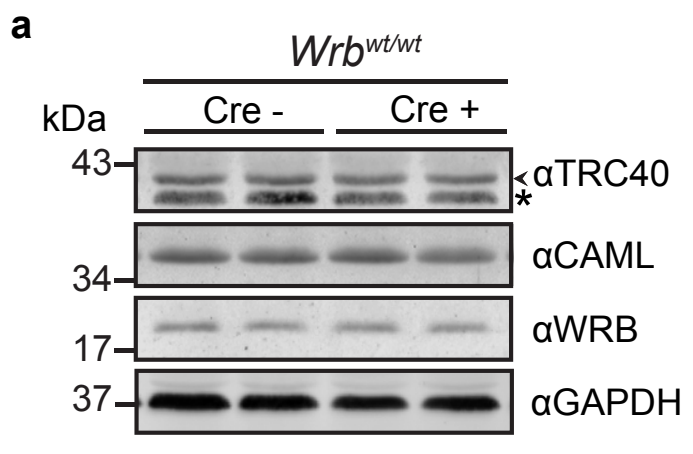

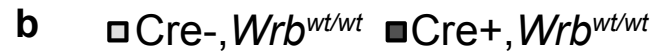

C

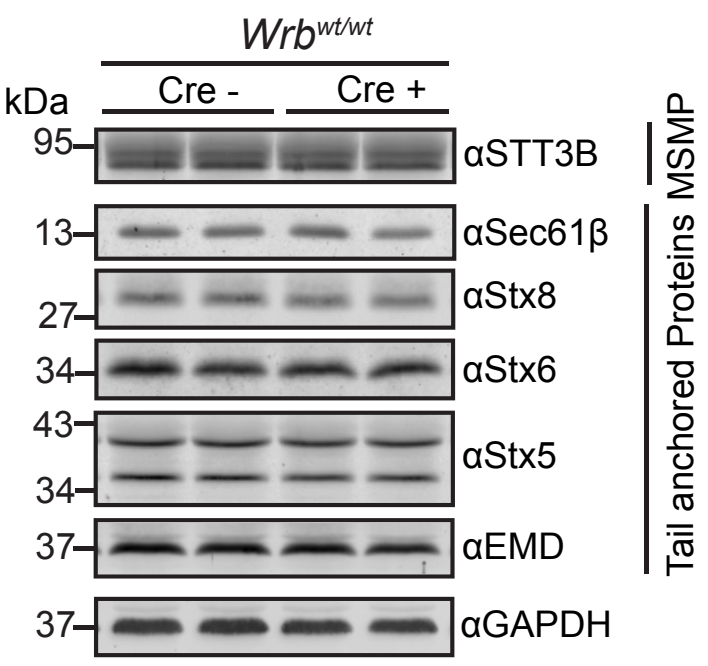

d

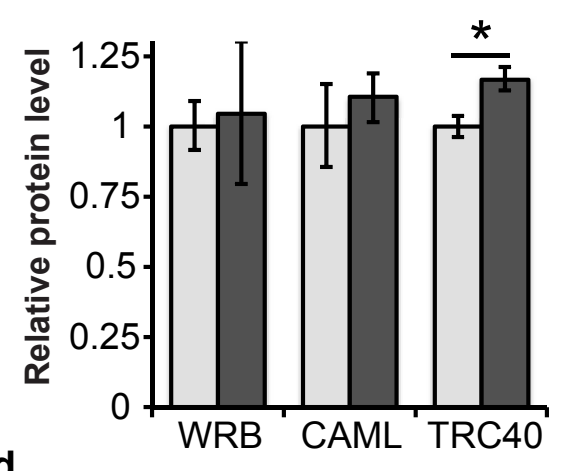

口Cre-,Wrb ${ }^{\text {wt/wt }}$ aCre+,Wrb ${ }^{\text {wt } / w t}$

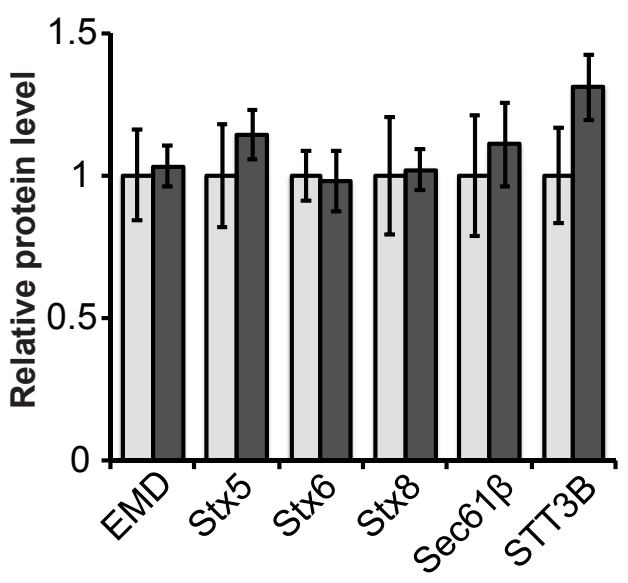

e

$W_{r} b^{w t / w t}$

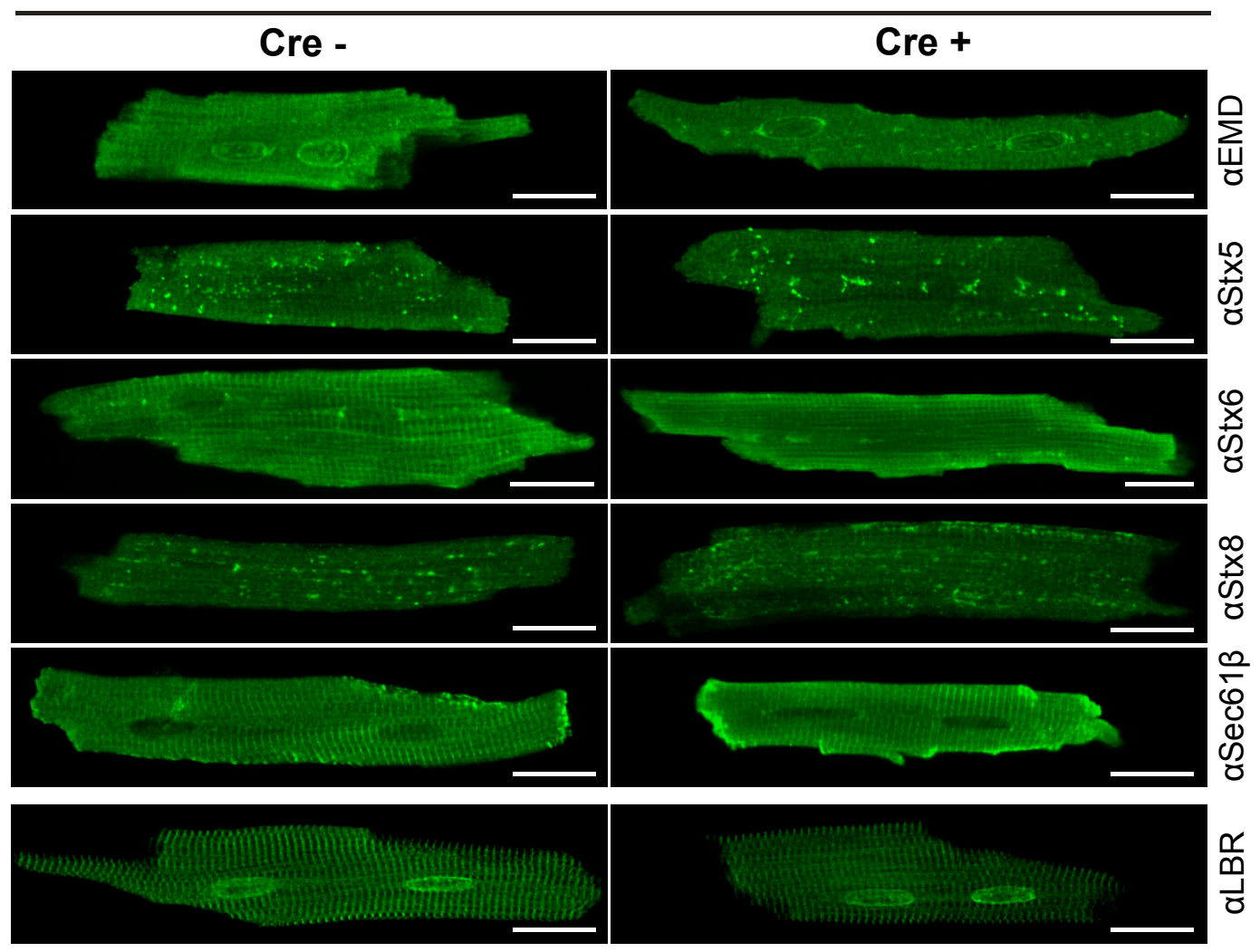


Supplementary Figure 3

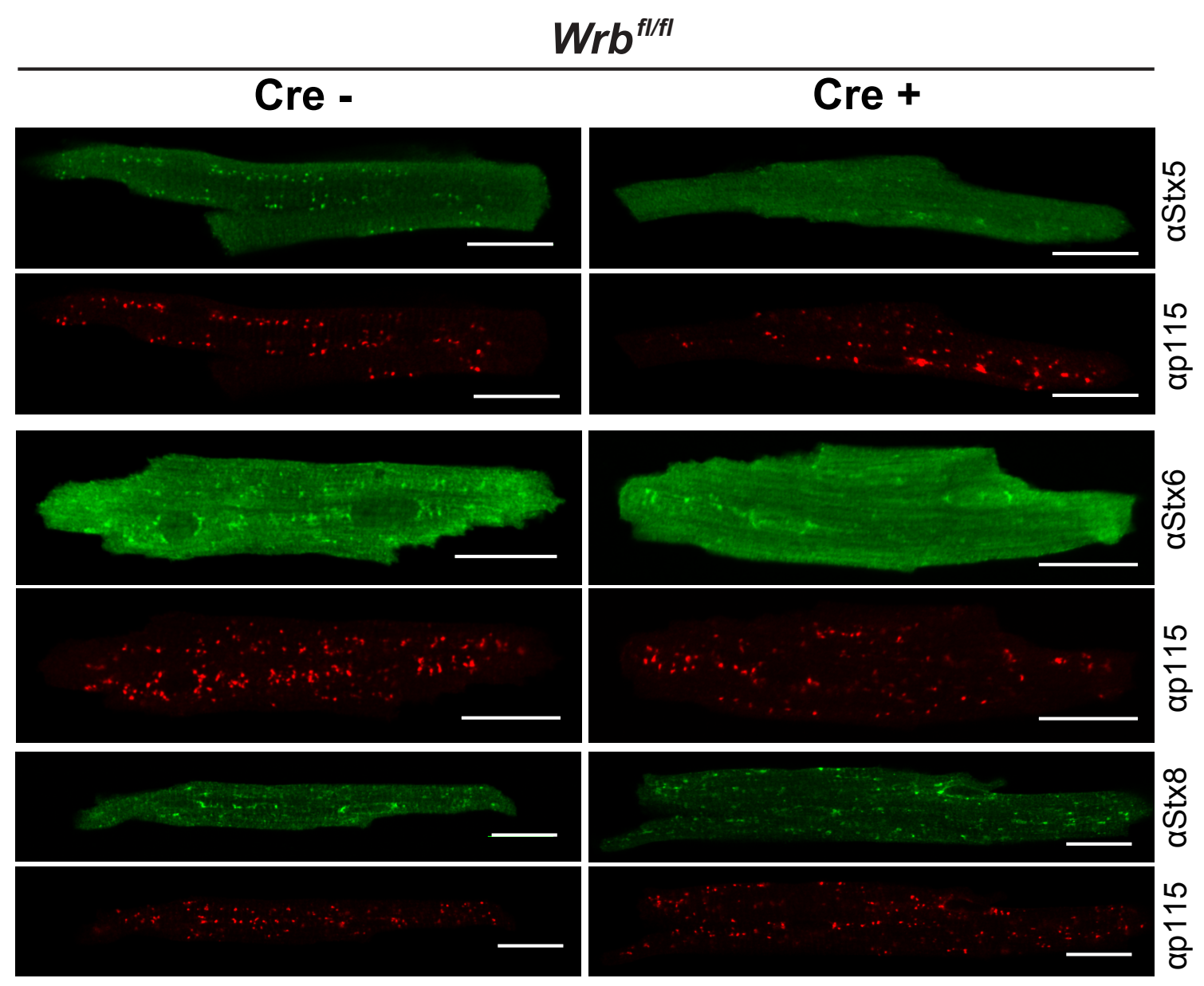




\section{Supplementary Figure 4}

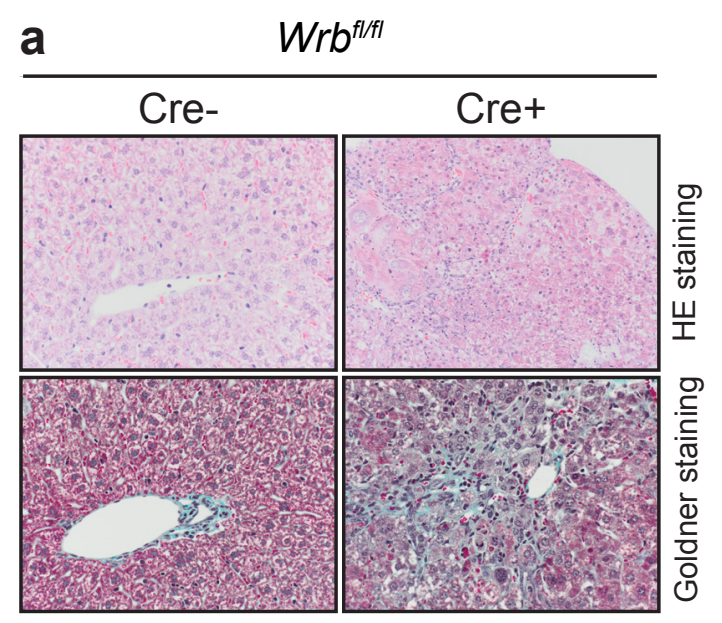

b

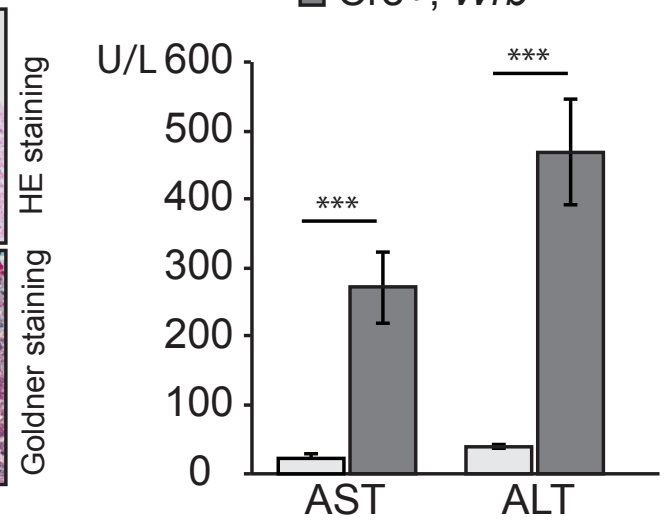

C

$\mathrm{mg} / \mathrm{dL} 80 \square \mathrm{Cre}+, W r b^{f / f t}$

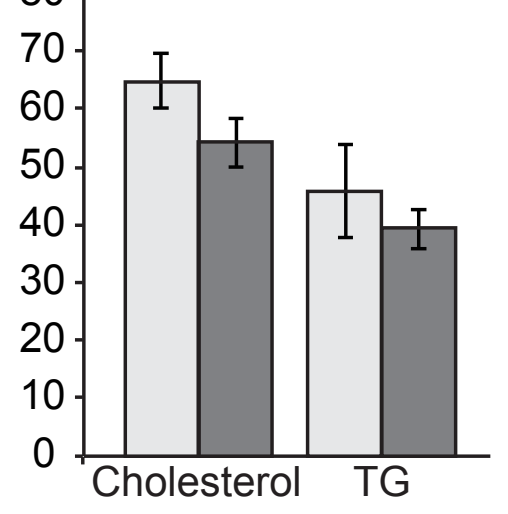

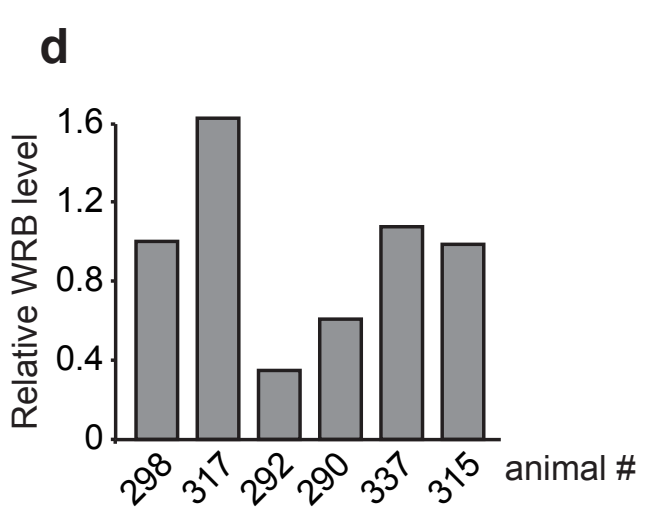
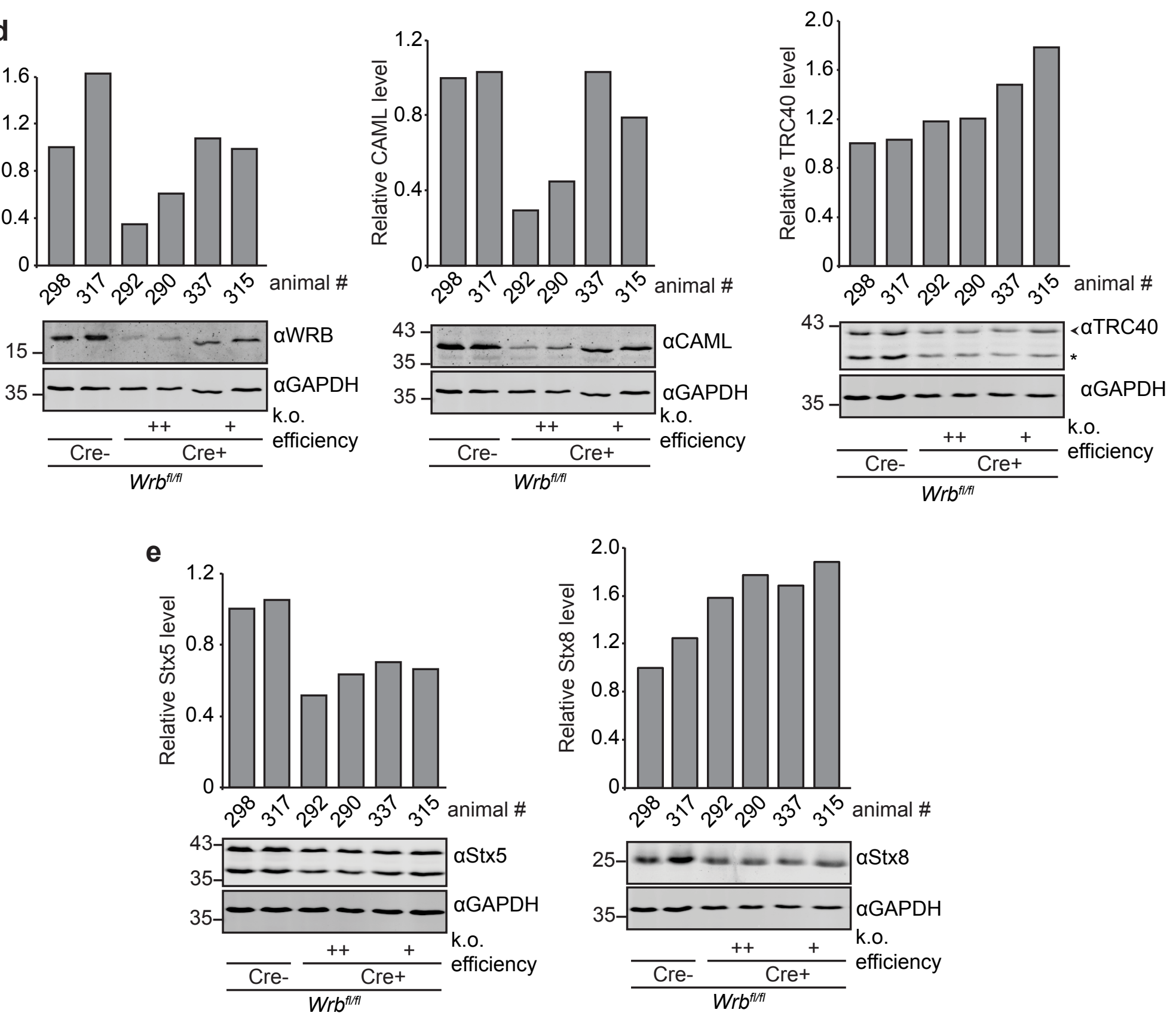


\section{Supplementary Figure 5}

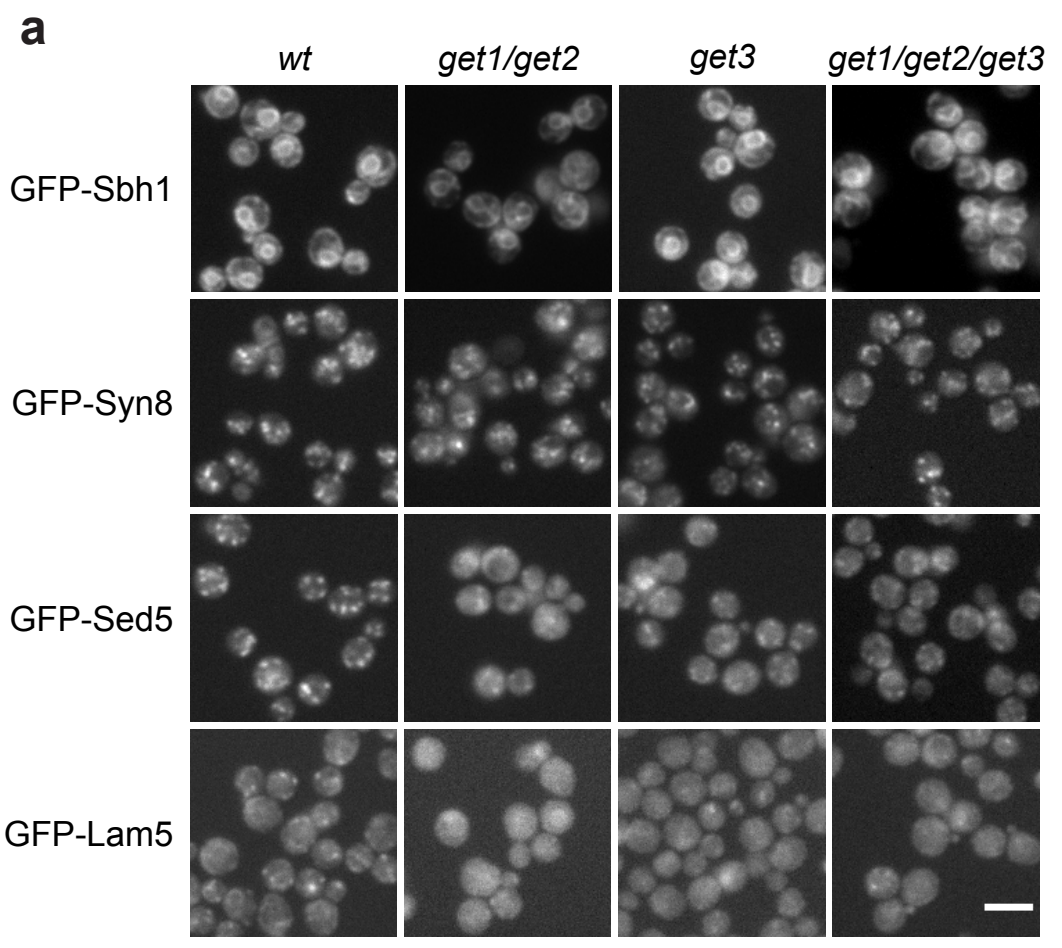

b
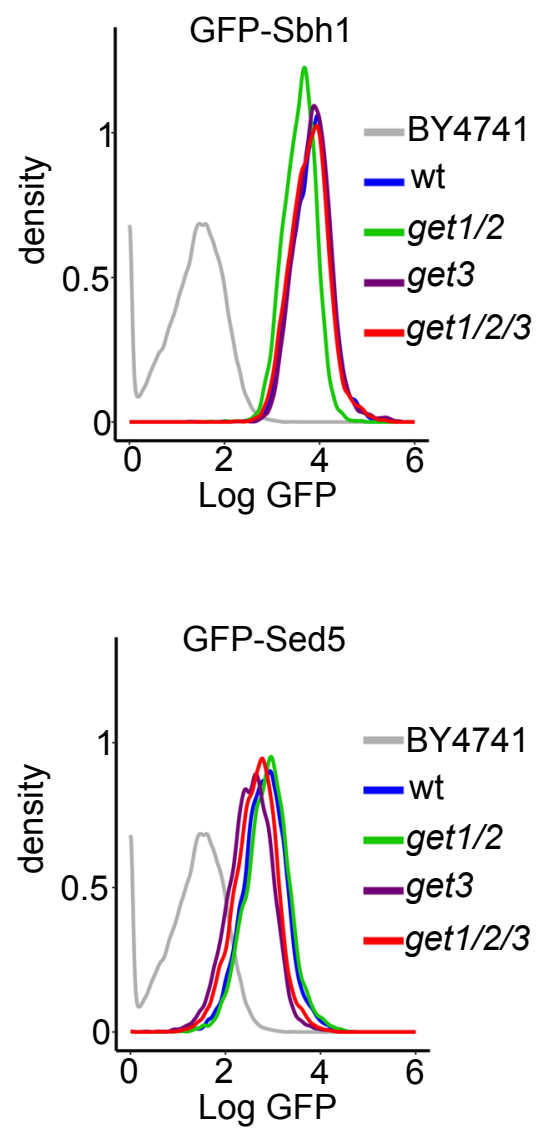
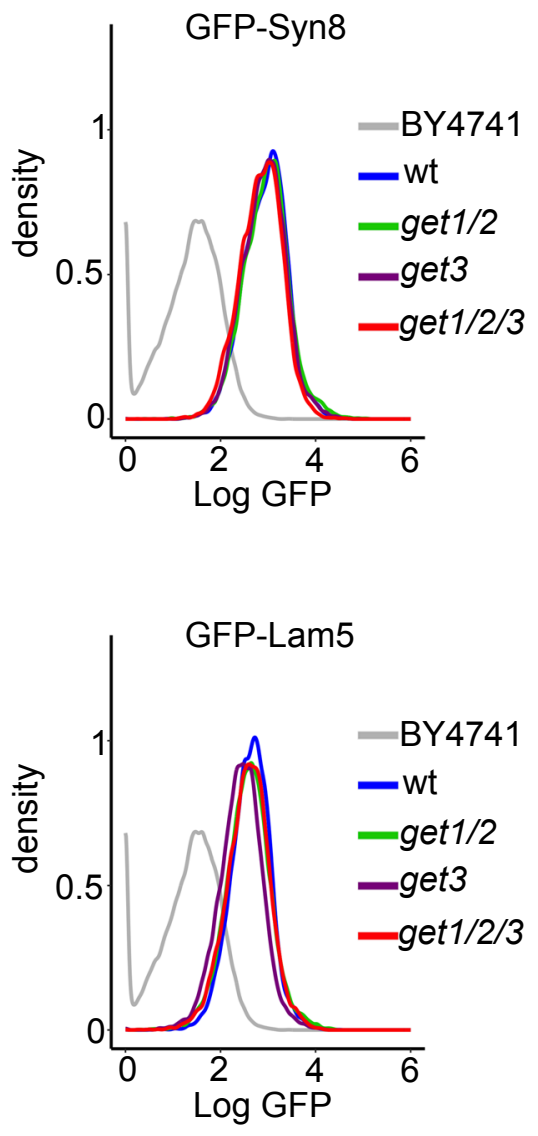

C

\begin{tabular}{|c|c|c|}
\hline Gene & $\begin{array}{c}\text { Localization in } \\
\text { SWAT library } \\
\end{array}$ & $\begin{array}{c}\text { Change in localization in get } 1 / \text { get } 2 \text {, } \\
\text { get } 3, \text { get } 1 / \text { get } 2 / \text { get } 3 \text { strains }\end{array}$ \\
\hline LAM5 & punctate, ER & partial loss of puncta and ER staining \\
\hline FMP32 & faint & mitochondria \\
\hline SED5 & punctate & partial loss of puncta \\
\hline BET1 & punctate & no visible change \\
\hline BOS1 & punctate & no visible change \\
\hline CYB5 & ER & no visible change \\
\hline FIS1 & mitochondria & no visible change \\
\hline GEM1 & mitochondria & no visible change \\
\hline GOS1 & punctate & no visible change \\
\hline HFD1 & ER & no visible change \\
\hline LAM6 & punctate & no visible change \\
\hline NYV1 & vacuole & no visible change \\
\hline PEP12 & punctate & no visible change \\
\hline $\mathrm{SBH} 1$ & ER & no visible change \\
\hline SCS2 & ER & no visible change \\
\hline SCS22 & cytosol & no visible change \\
\hline SEC20 & ER & no visible change \\
\hline SEC22 & ER & no visible change \\
\hline SFT1 & cytosol & no visible change \\
\hline SNC1 & punctate, cytosol & no visible change \\
\hline SNC2 & punctate & no visible change \\
\hline SSO1 & vacuole & no visible change \\
\hline $\mathrm{SSO} 2$ & cell periphery & no visible change \\
\hline SSS1 & ER & no visible change \\
\hline SYN8 & punctate & no visible change \\
\hline TLG1 & punctate & no visible change \\
\hline TLG2 & punctate & no visible change \\
\hline TOM7 & mitochondria & no visible change \\
\hline UBC6 & ER & no visible change \\
\hline USE1 & ER & no visible change \\
\hline VAM3 & vacuole & no visible change \\
\hline VPS64 & ER & no visible change \\
\hline VTI1 & punctate & no visible change \\
\hline YDL012C & bud tip, bud neck & no visible change \\
\hline YDR034W-B & cell periphery & no visible change \\
\hline YLR283W & ER & no visible change \\
\hline YSY6 & ER & no visible change \\
\hline FAR10 & faint & no visible change \\
\hline UFE1 & faint & no visible change \\
\hline YSP2 & faint & no visible change \\
\hline CoX16 & not in library & not in library \\
\hline HMX1 & not in library & not in library \\
\hline LAM4 & not in library & not in library \\
\hline $\mathrm{SBH} 2$ & not in library & not in library \\
\hline YDR210W & not in library & not in library \\
\hline
\end{tabular}

d kDa wt get1/2 get3 get1/2/3 $\alpha$ Sed5 $\alpha$ a

e

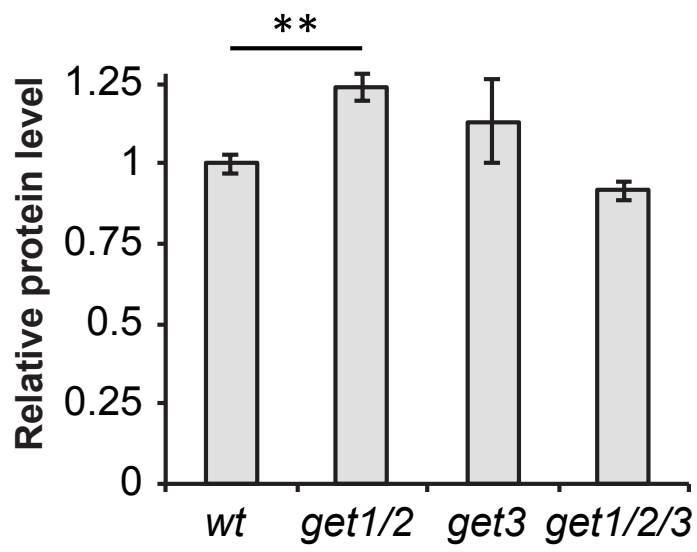




\section{Supplementary Figure 6}

a

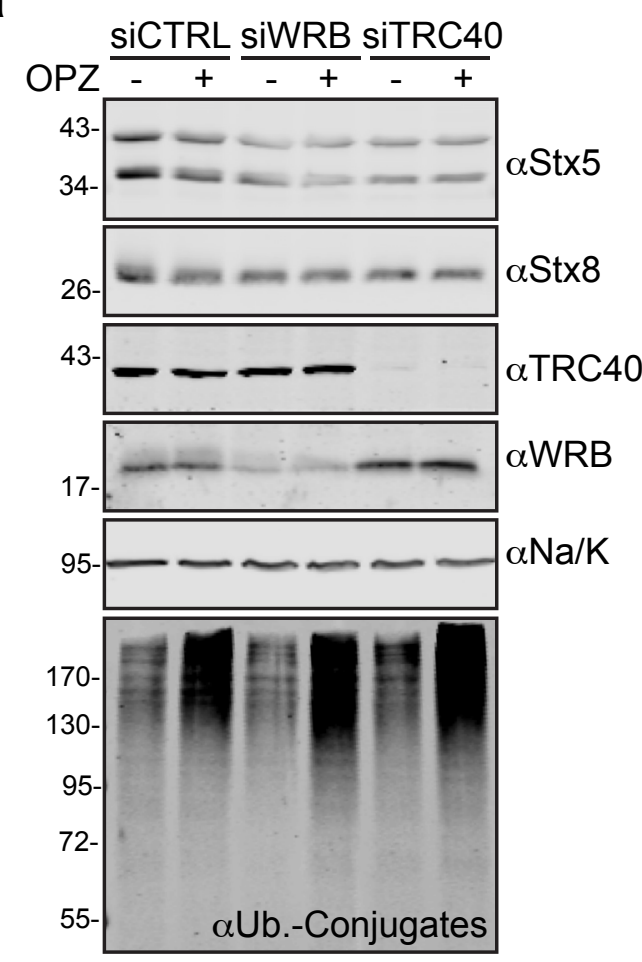

d

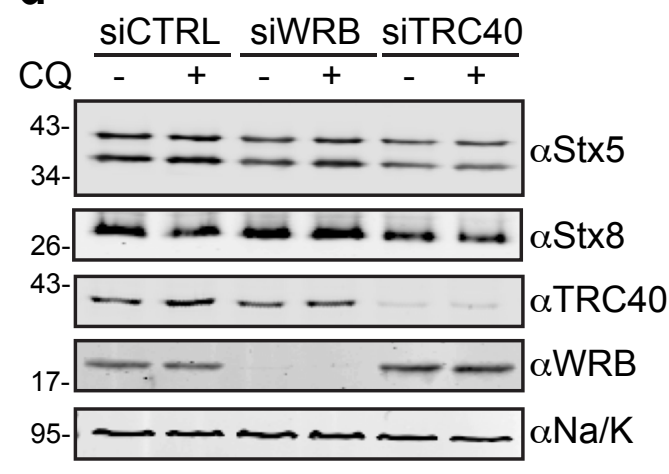

f

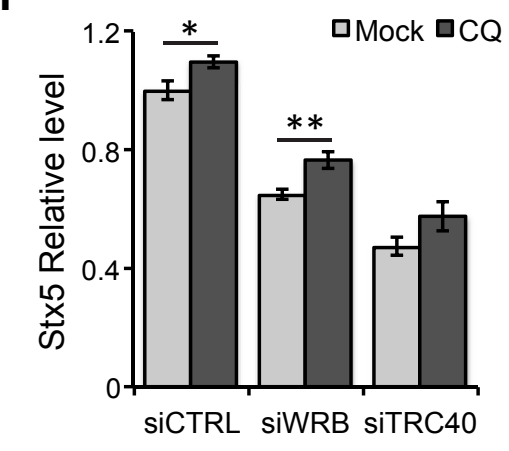

b

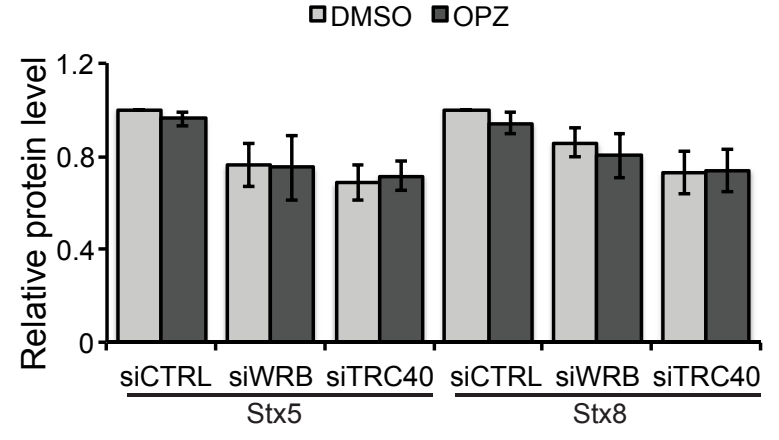

C

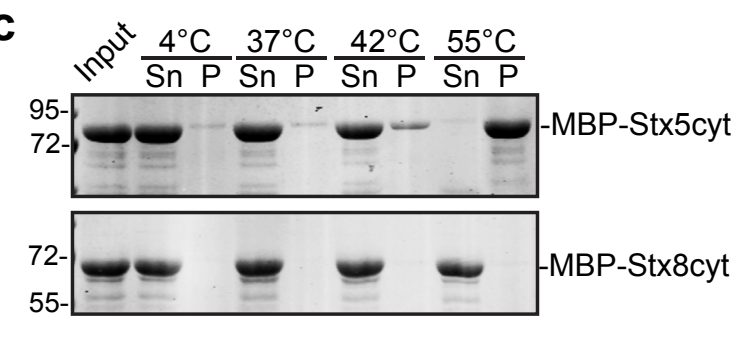

e

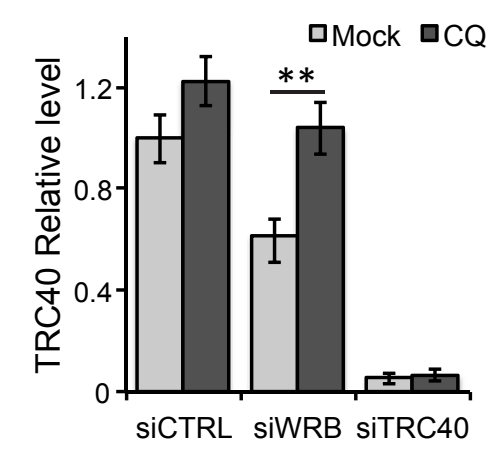

g

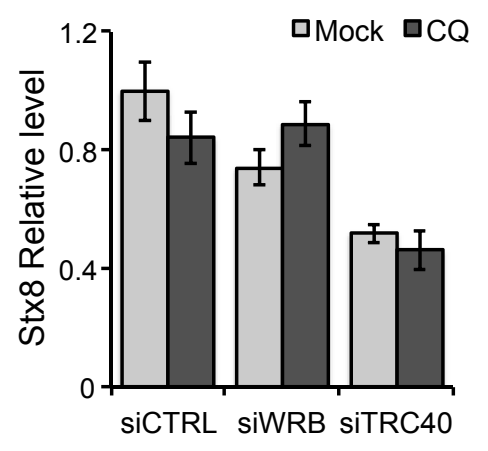




\section{Discussion}

In order to investigate the role of the TRC40 pathway in heart physiology and disease development, a cardiomyocyte-specific, tamoxifen inducible knockout of WRB was generated by crossing two mouse lines: (i) a MerCreMer recombinase line under the control of the cardiac Myh6 promoter (from Jackson Laboratory) and (ii) a previously described $w r b^{f / f f l}$ line, where exons 2 to 4 in the wrb locus are flanked by LoxP recombination sites (Vogl et al., 2016). In parallel, a second mouse line was generated in order to control for putative unspecific effects due the MerCreMer recombinase rather than the depletion of WRB (manuscript 2).

I focused particularly on heart muscle due to the association of WRB with congenital heart disease in Down syndrome patients (Egeo et al., 1998) as well as the severe cardiac abnormalities observed in the constitutive WRB knockdown models in medaka fish (Murata et al., 2009) and Xenopus laevis (Sojka et al., 2014) embryos.

Although several molecular aspects of the TRC40 pathway have been described by in vitro approaches over the years, it is not known how the different TRC40 pathway components respond in vivo once the receptor function is impaired. In order to gain insights into this, the components TRC40 and CAML were evaluated by Western blot in cardiomyocytes from the WRB knockout and cre recombinase control lines. The analysis confirmed an efficient knockout of WRB in heart upon tamoxifen injection. Interestingly, CAML and TRC40 were also decreased in the knockout line (manuscript 2). RNA analysis of these components showed that TRC40 and CAML transcript levels are unaffected, suggesting that the effect of WRB depletion on these components occurs in a post-translational 
manner. In yeast, the deletion of Get1 or Get2 causes the decrease of the second receptor component (Mariappan et al., 2011; Stefer et al., 2011). Moreover, Colombo et al. (2016) recently observed that the silencing of WRB or CAML causes a reduction in the mRNA and protein levels of the other subunit in HeLa cells (Colombo et al., 2016). Nevertheless, a downregulation of CAML mRNA upon WRB depletion was not observed in the present study in cardiomyocytes or hepatocytes, suggesting that WRB might affect CAML protein turnover in those tissues (manuscript 2).

In order to gain insight into the mechanism of TRC40 protein turnover upon WRB depletion, experiments with inhibitors of the proteasome (oprozomib) and lysosomal function (chloroquine) were carried out in HeLa cells. While inhibition of poteasomal protein degradation had no effect, the inhibition of lysosomes leaded to the recovery of TRC40 levels (manuscript 2). This result suggests that autophagy is involved in the clearance of TRC40 in the absence of its receptor. It is worth to highlight that these findings are completely in line with results by Colombo et al. (2016), where a reduction of TRC40 by depleting CAML and WRB was also observed, although the turnover mechanism was not evaluated. It is possible to speculate that the degradation of TRC40 might allow the recognition and targeting of nascent TA proteins by alternative targeting pathways, favouring the their normal membrane insertion.

Taking into account that little is known about the spectrum of substrates that depend on TRC40 pathway, the fate of several endogenous TA proteins (i.e. syntaxins $-5,-6$ and -8 , emerin and Sec61 $\beta$ ) upon depletion of the TRC40 receptor was analysed by Western blot and indirect immunofluorescence (manuscript 2). Among them, syntaxin-5 (Stx5) isoforms ( $35 \mathrm{kDa}$ and $42 \mathrm{kDa}$ ) and emerin (also known as EMD, shown to be able to use the TRC40 pathway in manuscript 1) were significantly decreased in the WRB knockout animals. Stx5, known as Sed5 in yeast, is an essential Qa-SNARE involved in traffic within the Golgi apparatus and 
between Golgi and the ER (Hardwick and Pelham, 1992; Jahn and Scheller, 2006). Early yeast two-hybrid screens identified Sed5 as a prominent client of the GET system that requires Get3 and the Get1/Get2 receptor for its proper biogenesis (Schuldiner et al., 2008). In mammals, Norlin et al. (2015) showed that loss of TRC40 in $\beta$-cells leads to reduction of Stx5 and other TA proteins involved in Golgi-to-ER transport. These results show that the dependency of Stx5/Sed5 on the TRC40/GET pathway is conserved from yeast to mammals. Our results published in manuscript 2 confirm this conservation and highlight that the strong dependence on the TRC40 pathway is a unique feature of a small number of TA proteins including Stx5/Sed5.

In contrast, the steady state protein levels or the subcellular distribution of Sec61 $\beta$, an auxiliary component of the ER translocon and previously considered a model client of the TRC40 pathway based on in vitro insertion assays, and Syntaxin 8 (Stx8), a Qc-SNARE in the endosomal membrane system and highly expressed in heart (Kasai and Akagawa, 2001; Subramaniam et al., 2000), were not significantly affected in the cardiac WRB knockout line. Moreover, a Qbc SNARE Syntaxin 6 (Stx6), involved in vesicular traffic in the trans-Golgi network (Mallard et al., 2002), presented a change in subcellular localization without alteration of steady-state protein levels (manuscript 2).

To further investigate our finding that TRC40 pathway dependence is not a general feature of all TA proteins in vivo, a microscopy-based yeast screen was carried out in our lab on a larger number of TA proteins. A sub-library of $46 \mathrm{~N}$-terminally GFP-Tagged TA proteins was analysed by Ákos Farkas and Anne Clancy from the recently generated SWAp-Tag library (Yofe et al., 2016) based on the fact that they have human homologues (manuscript 2). This approach revealed that only two TA proteins, the well characterized Sed5 and Lam5, a protein involved in sterol transfer at membrane contact site (Gatta et al., 2015) are severely 
affected in terms of subcellular localization by deletion of GET system components (get3, get1,2 and get1,2,3 knockouts).

Our results in mammalian cells and yeast show that there is no correlation between the hydrophobicity of the TA protein TMDs and the dependency on TRC40/GET pathways in vivo (manuscript 2), as can be expected from previous in-vitro studies with model TA proteins (Favaloro et al., 2010; Jonikas et al., 2009; Leznicki et al., 2010; Rabu et al., 2008; Schuldiner et al., 2008; Stefanovic and Hegde, 2007). In order to understand the role of the TRC40 pathway in the biogenesis of TA proteins, the ER membrane insertion of Stx5 and Stx8, one strongly and one less affected substrate respectively, was analysed in vitro in TRC40-depleted reticulocyte lysates. Interestingly, the insertion of both TA proteins into ER-derived membranes was impaired in a considerable but not in a total manner by TRC40 depletion, suggesting that in this system TRC40 contributes substantially but not exclusively to their targeting. Based on these results, we hypothesize that alternative pathways/components such as $\mathrm{Hsp} 40 / \mathrm{Hsc70}$ (Rabu et al., 2008) or the recently described SND components (Aviram et al., 2016) might effectively take over the targeting of TA proteins like Stx8 or Sec61 $\beta$ but not TA proteins like Stx5 once the TRC40 pathway is impaired.

The RNA analysis of TA proteins in cardiomyocytes showed that there is no effect on the steady-state levels of mRNAs encoding the investigated TA proteins in the WRB knockout line (manuscript 2), suggesting that the effect on Stx5 or EMD proteins is a consequence of a post-transcriptional event, probably a result of an increased in the turnover of those proteins in the KO. Experiments with proteasomal and lysosomal inhibitors in HeLa cells showed that the knockout of TRC40 or WRB promotes the degradation Stx5 in the lysosomes and not in proteasomes. Previous invitro studies have shown that the Bag6 pretargeting complex actively mediates the ubiquitylation and subsequent proteasomal degradation of 
TA proteins (i.e. Sec61 $\beta$ ) that fail to engage TRC40 (Hessa et al., 2011). Additionally, MSP1, a mitochondrial/peroxisomal AAA-ATPase also present in mammals (ATAD1) mediates the clearance of mislocalized tailanchored in mitochondria once the GET pathway is impaired, through a mechanism that presumably involves the proteasome (Chen et al., 2014; Okreglak and Walter, 2014). However, our results with Stx5 in vivo clearly demonstrate that not all the TA proteins are targeted to proteasomal degradation in the absence of TRC40 or WRB. Considering that Bag6 is not present in yeast and that MSP1 is thought to act exclusively at the outer mitochondrial membrane and possibly at peroxisomal membranes, the existence of an additional clearing mechanism that relies on lysosomal degradation is not surprising.

In order to confirm a direct role of autophagy in the degradation of tail anchored proteins during their biogenesis, we performed siRNA-mediated downregulation in HeLa cells of FAM134B and p62/SQSTM, autophagy cargo receptors involved in selective autophagy of ER membrane "ERphagy" (Khaminets et al., 2015) or cytosolic aggregates (Pankiv et al., 2007), respectively (manuscript 2). Down-regulation of FAM134B lead to a significant recovery of Stx 5 levels in cells where TRC40 was also depleted. Previous studies have shown that FAM134B is an evolutionarily conserved component that is able to regulate the turnover and structure of the ER and possible the Golgi apparatus (Khaminets et al., 2015; Mochida et al., 2015). Moreover, the role of FAM134B in ER-phagy relies on two features of the protein: a reticulon domain, which promotes membrane remodelling, and an LC3-interacting-region (LIR motif) that is able to target ER fragments to autophagosomes via LC3-like modifiers.

Because we observed an effect of downregulating ER-phagy on the stability of Stx5 in the WRB knockout cells our results suggest that the degradation of Stx 5 upon TRC40 depletion occurs after its insertion into the ER membrane (manuscript 2), implying that alternative pathways 
might also operate during Stx5 biogenesis in the absence of the TRC40 system (Fig. 6). However, it is important to highlight that the delivery of Stx 5 to the ER membrane by TRC40 reduces Stx 5 turnover by autophagy, suggesting that TRC40 might perform additional roles during Stx5 biogenesis. For instance, apart from Stx5 TMD shielding, TRC40 might prevent Stx5 misfolding in its $\mathrm{N}$-terminal, cytoplasmic domain until delivery to the membrane has occurred.

Manuscript 1 provides a fundamental characterization of emerin as a TRC40-dependent TA protein client. This information was essential for the experiments performed in manuscript 2 where we were able to confirm emerin as a TRC40 pathway-dependent TA protein client in vivo. Furthermore, the work on emerin allowed us to study TA proteins of the inner nuclear membrane. Emerin is an ubiquitous serine-rich highly phosphorylated TA protein of the nuclear envelope, tightly associated with the nuclear lamina. Mutations of emerin gene $(E M D)$ cause recessive $X-$ linked Emery-Dreifuss Muscular Dystrophy (EDMD) (Bione et al., 1994), a neuromuscular disease characterized by weakening of specific skeletal muscles, contractures, cardiomyopathy, and cardiac conduction system defects that can cause sudden death (Ellis et al., 1998; Wulff et al., 1996). 


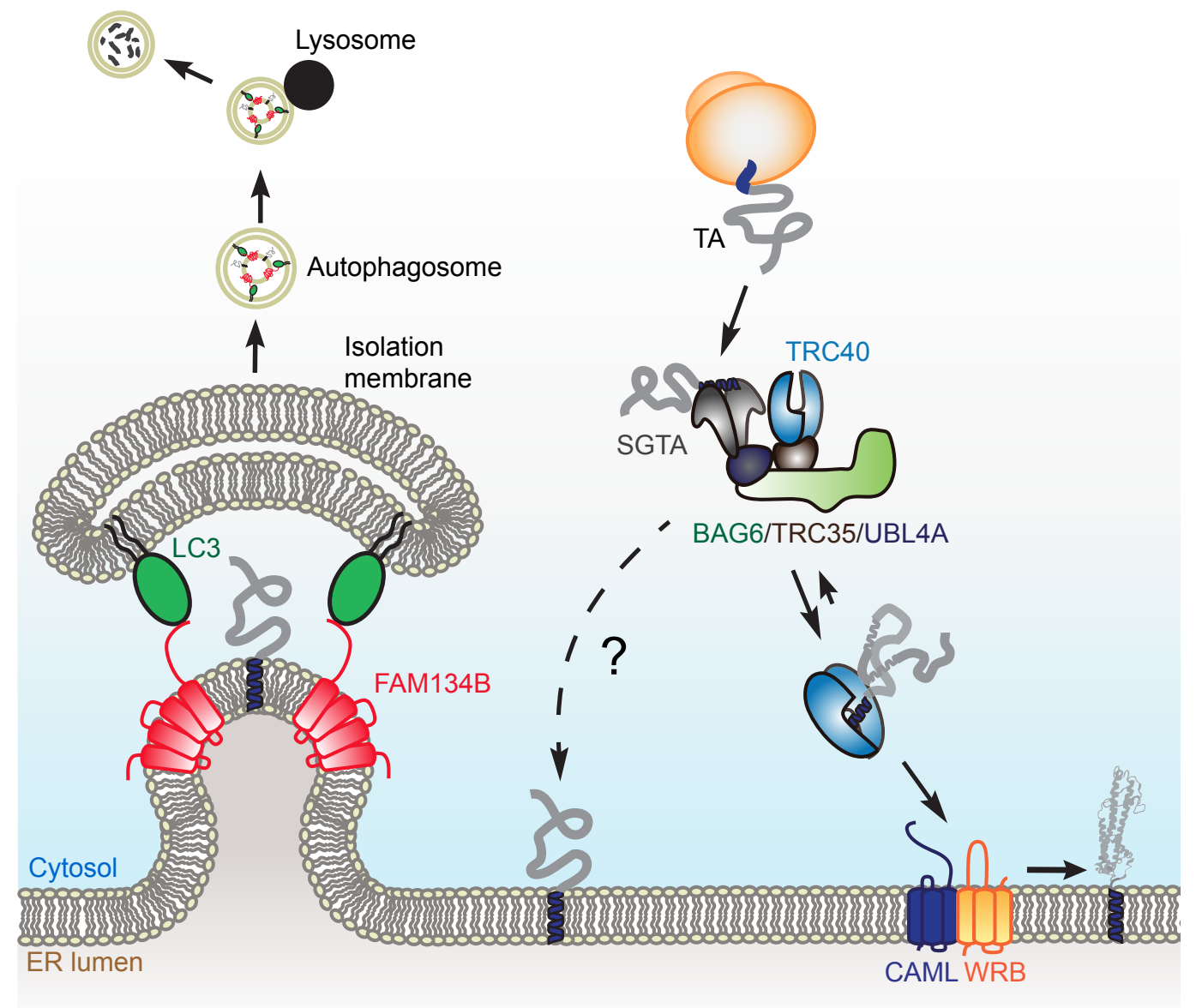

Figure 6. Model of the Integration of the TRC40 pathway into the cellular proteostasis network in vivo. In normal conditions, TRC40 targets nascent TA proteins to the WRB/CAML receptor at the ER. In the absence of the pathway, although the vast majority of TA proteins can still reach the membrane by alternative pathways, a subset of them are degraded by autophagy, in a FAM134B-dependent manner.

We confirmed that emerin is a client of the TRC40 pathway by in vitro and in vivo approaches (manuscript 1). First of all, recombinant emerin and TRC40 could be co-purified in a stable complex. Second, the integration of emerin in ER-enriched membranes depended on TRC40 and ATP presence and could be abolished by the addition of the cytosolic domains of WRB and CAML, suggesting that the ER receptor is also involved. Third, experiments with TRC40-depleted HeLa cells (manuscript 1) and in 
our WRB KO cardiomyocytes (manuscript 2) demonstrated that the protein is affected in terms of localization when the pathway is disrupted.

A subset of EDMD-derived mutations within the region that contains emerin TMD was also analyzed. As expected, emerin mutants with drastic changes within the TMD localized to the cytosol in HeLa and displayed a strong reduction in terms of binding to TRC40 and membrane insertion. Interestingly, the Pro183Thr and Pro183His variants presented a significant reduction in membrane insertion and were prone to form cytoplasmic aggregates, even thought their TMDs are intact. The results with the Pro183 mutants are valuable as there are no reports describing effects of TA protein cytoplasmic domains on integration efficiency. This finding raises the possibility that regions upstream the TA-protein TMD could affect the interaction with TRC40.

In collaboration with Janine Pfaff, the targeting of the different emerin mutants to the INM was evaluated by two independent approaches: (i) differential membrane permeabilization in cells transfected with the different mutants and (ii) rapamycin-induced dimerization of an FRB domain tagged to the emerin mutants with a nucleoplasmic GFP-tagged version of the FKBP12 protein. Both methodologies showed that emerin Pro183 mutants and the Phe240His-FS mutant present an impaired targeting to the INM, even though these variants can still reach the outer nuclear membrane and present similar staining patters as wild type emerin when analysed by indirect immunofluorescence (manuscript 1). Intriguingly, the insertion efficiency of the Phe240His-FS mutant via the TRC40 pathway was markedly low, raising the possibility that the pathway affects the correct targeting of emerin to its final destination. 


\section{Conclusions and perspectives}

This thesis demonstrates a role of the TRC40 system during the insertion of emerin, the first described TA protein of the INM that requires the pathway. Moreover, the analysis of emerin disease mutants shows that there are membrane insertion differences and evidence of mislocalization, phenomena that could contribute to the pathological phenotype occurring in some EDMD patients.

The thesis also provides evidence for a role of the TRC40 pathway in the cellular proteostasis network in vivo. First, I found that the depletion of WRB in heart tissue leads to a significant decrease at steady state level of other components of the pathway, namely CAML and TRC40. It was demonstrated that in the case of TRC40, this reduction is due to an increase in its turnover by lysosomal degradation. Unlike a previous report in HeLa cells (Colombo et al., 2016), depletion of WRB in heart tissue had no effect in the mRNA levels of CAML. However, further studies addressing the causes of TRC40 and CAML reduction are required, in order to test whether such a reduction allow the targeting of TA proteins by alternative pathways/components (e.g. HSP40/HSC70 pathway or SND components). Second, I observed that a subset of TA proteins (i.e. Stx5, Stx6, emerin) is affected in their steady-state levels and their subcellular localization upon WRB knockout in ventricular cardiomyocytes.

It is important to highlight that a correlation between hydrophobicity of TA protein TMDs and TRC40 dependency was not observed when comparing the steady state levels and subcellular localization of the endogenous TA proteins evaluated. These findings in mammalian cells were also supported by a microscopic screen in yeast for GET-dependent TA proteins carried out by our lab. Based on the low number of TRC40dependent TA protein clients identified, it is possible that alternative 
pathways/components substitute the function of the TRC40 pathway as has been previously proposed (Aviram et al., 2016; Rabu et al., 2008), thus targeting the majority of TA proteins to the ER membrane. Nevertheless, my work delineates TA protein clients such as Stx5 or emerin as critically dependent on the TRC40 pathway for their stability and correct targeting. Based on these results it will be possible in the future to elucidate the relative physiological contributions of different membrane protein targeting pathways.

With respect to the integration of the TRC40 pathway into the cellular proteostasis network, the work presented in this thesis provides evidence of a preponderant role of FAM134B in the clearance of TA proteins by selective autophagy once the TRC40-pathway is impaired. Therefore, together with Bag6/RNF126 and ATAD1, FAM134B contributes to TA protein quality control and cellular proteostasis. However, in the future it will be important to clarify the way TA-proteins are recognized and degraded by selective autophagy. 


\section{References}

Abell, B. M., Pool, M. R., Schlenker, O., Sinning, I. and High, S. (2004). Signal recognition particle mediates post-translational targeting in eukaryotes. The EMBO Journal 23, 2755-2764.

Abell, B. M., Rabu, C., Leznicki, P., Young, J. C. and High, S. (2007). Post-translational integration of tail-anchored proteins is facilitated by defined molecular chaperones. J Cell Sci 120, 1743-1751.

Akahane, T., Sahara, K., Yashiroda, H., Tanaka, K. and Murata, S. (2013). Involvement of Bag6 and the TRC pathway in proteasome assembly. Nat Commun 4, 2234.

Amsterdam, A., Nissen, R. M., Sun, Z., Swindell, E. C., Farrington, S. and Hopkins, N. (2004). Identification of 315 genes essential for early zebrafish development. Proc. Natl. Acad. Sci. U.S.A. 101, 1279212797.

Ast, T., Cohen, G. and Schuldiner, M. (2013). A Network of Cytosolic Factors Targets SRP-Independent Proteins to the Endoplasmic Reticulum. Cell 152, 1134-1145.

Auld, K. L., Hitchcock, A. L., Doherty, H. K., Frietze, S., Huang, L. S. and Silver, P. A. (2006). The Conserved ATPase Get3/Arr4 Modulates the Activity of Membrane-Associated Proteins in Saccharomyces cerevisiae. Genetics 174, 215-227.

Aviram, N. and Schuldiner, M. (2014). Embracing the void--how much do we really know about targeting and translocation to the endoplasmic reticulum? Curr Opin Cell Biol 29, 8-17.

Aviram, N., Ast, T., Costa, E. A., Arakel, E. C., Chuartzman, S. G., Jan, C. H., Hassenteufel, S., Dudek, J., Jung, M., Schorr, S., et al. (2016). The SND proteins constitute an alternative targeting route to the endoplasmic reticulum. Nature 540, 134-.

Battle, A., Jonikas, M. C., Walter, P., Weissman, J. S. and Koller, D. (2010). Automated identification of pathways from quantitative genetic interaction data. Mol Syst Bio/ 6, -379.

Benjamin, E. J., Blaha, M. J., Chiuve, S. E., Cushman, M., Das, S. R., Deo, R., de Ferranti, S. D., Floyd, J., Fornage, M., Gillespie, C., et al. (2017). Heart Disease and Stroke Statistics-2017 Update: A Report From the American Heart Association. Circulation. 
Bione, S. S., Maestrini, E. E., Rivella, S. S., Mancini, M. M., Regis, S. S., Romeo, G. G. and Toniolo, D. D. (1994). Identification of a novel $\mathrm{X}$-linked gene responsible for Emery-Dreifuss muscular dystrophy. Nat Genet 8, 323-327.

Blobel, G. and Dobberstein, B. (1975a). Transfer to proteins across membranes. II. Reconstitution of functional rough microsomes from heterologous components. J. Cell Biol. 67, 852-862.

Blobel, G. and Dobberstein, B. (1975b). Transfer of proteins across membranes. I. Presence of proteolytically processed and unprocessed nascent immunoglobulin light chains on membrane-bound ribosomes of murine myeloma. J. Cell Biol. 67, 835-851.

Borgese, N. and Fasana, E. (2011). Targeting pathways of C-tailanchored proteins. Biochim. Biophys. Acta 1808, 937-946.

Boskovic, J., Soler-Mira, A., García-Cantalejo, J. M., Ballesta, J. P., Jiménez, A. and Remacha, M. (1996). The sequence of a 16,691 bp segment of Saccharomyces cerevisiae chromosome IV identifies the DUN1, PMT1, PMT5, SRP14 and DPR1 genes, and five new open reading frames. Yeast 12, 1377-1384.

Bryda, E. C., Johnson, N. T., Ohlemiller, K. K., Besch-Williford, C. L., Moore, E. and Bram, R. J. (2012). Conditional deletion of calciummodulating cyclophilin ligand causes deafness in mice. Mamm. Genome 23, 270-276.

Chacinska, A., Koehler, C. M., Milenkovic, D., Lithgow, T. and Pfanner, N. (2009). Importing mitochondrial proteins: machineries and mechanisms. Cell 138, 628-644.

Chang, Y.-W., Chuang, Y.-C., Ho, Y.-C., Cheng, M.-Y., Sun, Y.-J., Hsiao, C.-D. and Wang, C. (2010). Crystal structure of Get4-Get5 complex and its interactions with Sgt2, Get3, and Ydj1. J Biol Chem 285, 9962-9970.

Chang, Y.-W., Lin, T.-W., Li, Y.-C., Huang, Y.-S., Sun, Y.-J. and Hsiao, C.-D. (2012). Interaction surface and topology of Get3-Get4-Get5 protein complex, involved in targeting tail-anchored proteins to endoplasmic reticulum. J Biol Chem 287, 4783-4789.

Chaoui, R., Heling, K. S., Sarioglu, N. and Schwabe, M. (2005). Aberrant right subclavian artery as a new cardiac sign in second- and third-trimester fetuses with Down syndrome. American journal of ....

Chartron, J. W., Clemons, W. M. and Suloway, C. J. M. (2012). The complex process of GETting tail-anchored membrane proteins to the ER. Curr Opin Struct Biol 22, 217-224. 
Chartron, J. W., Suloway, C. J. M., Zaslaver, M. and Clemons, W. M. J. (2010). Structural characterization of the Get4/Get5 complex and its interaction with Get3. Proc. Natl. Acad. Sci. U.S.A. 107, 12127-12132.

Chen, Y. C., Umanah, G. K. E., Dephoure, N., Andrabi, S. A., Gygi, S. P., Dawson, T. M., Dawson, V. L. and Rutter, J. (2014). Msp1/ATAD1 maintains mitochondrial function by facilitating the degradation of mislocalized tail-anchored proteins. The EMBO Journal 33, 1548-1564.

Colombo, S. F., Cardani, S., Maroli, A., Vitiello, A., Soffientini, P., Crespi, A., Bram, R. F., Benfante, R. and Borgese, N. (2016). Tailanchored protein biogenesis in mammals: function and reciprocal interactions of the two subunits of the TRC40 receptor. J Biol Chem.

Colombo, S. F., Longhi, R. and Borgese, N. (2009). The role of cytosolic proteins in the insertion of tail-anchored proteins into phospholipid bilayers. J Cell Sci 122, 2383-2392.

Daniele, L. L., Emran, F., Lobo, G. P., Gaivin, R. J. and Perkins, B. D. (2016). Mutation of wrb, a Component of the Guided Entry of TailAnchored Protein Pathway, Disrupts Photoreceptor Synapse Structure and Function. Invest. Ophthalmol. Vis. Sci. 57, 2942-2954.

Desmots, F., Russell, H. R., Lee, Y., Boyd, K. and McKinnon, P. J. (2005). The reaper-binding protein scythe modulates apoptosis and proliferation during mammalian development. Mol. Cell. Biol. 25, 10329-10337.

Desmots, F., Russell, H. R., Michel, D. and McKinnon, P. J. (2008). Scythe regulates apoptosis-inducing factor stability during endoplasmic reticulum stress-induced apoptosis. J Biol Chem 283, 3264-3271.

Egeo, A., Mazzocco, M., Sotgia, F., Arrigo, P., Oliva, R., Bergonòn, S., Nizetic, D., Rasore-Quartino, A. and Scartezzini, P. (1998). Identification and characterization of a new human cDNA from chromosome 21q22.3 encoding a basic nuclear protein. Hum. Genet. 102, 289-293.

Ellis, J. A. J., Craxton, M. M., Yates, J. R. J. and Kendrick-Jones, J. J. (1998). Aberrant intracellular targeting and cell cycle-dependent phosphorylation of emerin contribute to the Emery-Dreifuss muscular dystrophy phenotype. J Cell Sci 111 ( Pt 6), 781-792.

Favaloro, V., Spasic, M., Schwappach, B. and Dobberstein, B. (2008). Distinct targeting pathways for the membrane insertion of tail-anchored (TA) proteins. J Cell Sci 121, 1832-1840. 
Favaloro, V., Vilardi, F., Schlecht, R., Mayer, M. P. and Dobberstein, B. (2010). Asna1/TRC40-mediated membrane insertion of tailanchored proteins. J Cell Sci 123, 1522-1530.

Garg, S. G. and Gould, S. B. (2016). The Role of Charge in Protein Targeting Evolution. Trends in Cell Biology 26, 894-905.

Gatta, A. T., Wong, L. H., Sere, Y. Y., Calderón-Noreña, D. M., Cockcroft, S., Menon, A. K. and Levine, T. P. (2015). A new family of StART domain proteins at membrane contact sites has a role in ERPM sterol transport. Elife 4.

Giannopoulou, E.-A., Emmanouilidis, L., Sattler, M., Dodt, G. and Wilmanns, M. (2016). Towards the molecular mechanism of the integration of peroxisomal membrane proteins. Biochim. Biophys. Acta 1863, 863-869.

Goodship, J., Cross, I., LiLing, J. and Wren, C. (1998). A population study of chromosome 22q11 deletions in infancy. Arch. Dis. Child. 79, 348-351.

Grudnik, P., Bange, G. and Sinning, I. (2009). Protein targeting by the signal recognition particle. Biological Chemistry 390, 775-782.

Hardwick, K. G. and Pelham, H. R. (1992). SED5 encodes a 39-kD integral membrane protein required for vesicular transport between the ER and the Golgi complex. J. Cell Biol. 119, 513-521.

Hegde, R. S. and Keenan, R. J. (2011). Tail-anchored membrane protein insertion into the endoplasmic reticulum. Nat. Rev. Mol. Cell Biol. 12, 787-798.

Hessa, T., Sharma, A., Mariappan, M., Eshleman, H. D., Gutierrez, E. and Hegde, R. S. (2011). Protein targeting and degradation are coupled for elimination of mislocalized proteins. Nature 475, 394-397.

Huh, W.-K., Falvo, J. V., Gerke, L. C., Carroll, A. S., Howson, R. W., Weissman, J. S. and O'Shea, E. K. (2003). Global analysis of protein localization in budding yeast. Nat. Cell Biol. 425, 686-691.

Jahn, R. and Scheller, R. H. (2006). SNAREs - engines for membrane fusion. Nat. Rev. Mol. Cell Biol. 7, 631-643.

Johnson, N., Vilardi, F., Lang, S., Leznicki, P., Zimmermann, R. and High, S. (2012). TRC40 can deliver short secretory proteins to the Sec61 translocon. J Cell Sci 125, 3612-3620.

Jonikas, M. C., Collins, S. R., Denic, V., Oh, E., Quan, E. M., Schmid, V., Weibezahn, J., Schwappach, B., Walter, P., Weissman, J. S., et al. (2009). Comprehensive characterization of genes required for 
protein folding in the endoplasmic reticulum. Science 323, 1693-1697.

Kalbfleisch, T., Cambon, A. and Wattenberg, B. W. (2007). A bioinformatics approach to identifying tail-anchored proteins in the human genome. Traffic 8, 1687-1694.

Karkaria, C. E., Chen, C. M. and Rosen, B. P. (1990). Mutagenesis of a nucleotide-binding site of an anion-translocating ATPase. J Biol Chem 265, 7832-7836.

Kasai, K. and Akagawa, K. (2001). Roles of the cytoplasmic and transmembrane domains of syntaxins in intracellular localization and trafficking. J Cell Sci 114, 3115-3124.

Kaur, P. and Rosen, B. P. (1992). Mutagenesis of the C-terminal nucleotide-binding site of an anion-translocating ATPase. J Biol Chem 267, 19272-19277.

Khaminets, A., Heinrich, T., Mari, M., Grumati, P., Huebner, A. K., Akutsu, M., Liebmann, L., Stolz, A., Nietzsche, S., Koch, N., et al. (2015). Regulation of endoplasmic reticulum turnover by selective autophagy. Nature 522, 354-.

Krysztofinska, E. M., Martínez-Lumbreras, S., Thapaliya, A., Evans, N. J., High, S. and Isaacson, R. L. (2016). Structural and functional insights into the E3 ligase, RNF126. Sci Rep 6, 26433.

Kutay, U., Hartmann, E. and Rapoport, T. (1993). A class of membrane proteins with a C-terminal anchor. Trends in Cell Biology 3, 72-75.

Lee, M. J. and DohIman, H. G. (2008). Coactivation of G Protein Signaling by Cell-Surface Receptors and an Intracellular Exchange Factor. Current Biology 18, 211-215.

Leznicki, P. and High, S. (2012). SGTA antagonizes BAG6-mediated protein triage. Proc. Natl. Acad. Sci. U.S.A. 109, 19214-19219.

Leznicki, P., Clancy, A., Schwappach, B. and High, S. (2010). Bat3 promotes the membrane integration of tail-anchored proteins. J Cell Sci 123, 2170-2178.

Leznicki, P., Korac-Prlic, J., Kliza, K., Husnjak, K., Nyathi, Y., Dikic, I. and High, S. (2015). Binding of SGTA to Rpn13 selectively modulates protein quality control. J Cell Sci 128, 3187-3196.

Leznicki, P., Roebuck, Q. P., Wunderley, L., Clancy, A., Krysztofinska, E. M., Isaacson, R. L., Warwicker, J., Schwappach, B. and High, S. (2013). The association of BAG6 with SGTA and tail-anchored proteins. PLOS ONE 8, e59590. 
Lin, S.-Y., Vollrath, M. A., Mangosing, S., Shen, J., Cardenas, E. and Corey, D. P. (2016). The zebrafish pinball wizard gene encodes WRB, a tail-anchored-protein receptor essential for inner-ear hair cells and retinal photoreceptors. J. Physiol. (Lond.) 594, 895-914.

Mallard, F., Tang, B. L., Galli, T., Tenza, D., Saint-Pol, A., Yue, X., Antony, C., Hong, W. J., Goud, B. and Johannes, L. (2002). Early/recycling endosomes-to-TGN transport involves two SNARE complexes and a Rab6 isoform. J. Cell Biol. 156, 653-664.

Mariappan, M., Li, X., Stefanovic, S., Sharma, A., Mateja, A., Keenan, R. J. and Hegde, R. S. (2010). A ribosome-associating factor chaperones tail-anchored membrane proteins. Nature 466, 1120 1124.

Mariappan, M., Mateja, A., Dobosz, M., Bove, E., Hegde, R. S. and Keenan, R. J. (2011). The mechanism of membrane-associated steps in tail-anchored protein insertion. Nature 477, 61-U69.

Mateja, A., Paduch, M., Chang, H. Y., Szydlowska, A., Kossiakoff, A. A., Hegde, R. S. and Keenan, R. J. (2015). Structure of the Get3 targeting factor in complex with its membrane protein cargo. Science 347, 1152-1155.

Mochida, K., Oikawa, Y., Kimura, Y., Kirisako, H., Hirano, H., Ohsumi, Y. and Nakatogawa, H. (2015). Receptor-mediated selective autophagy degrades the endoplasmic reticulum and the nucleus. Nature 522, 359-362.

Mock, J.-Y., Chartron, J. W., Zaslaver, M., Xu, Y., Ye, Y. and Clemons, W. M. (2015). Bag6 complex contains a minimal tail-anchor-targeting module and a mock BAG domain. Proc. Natl. Acad. Sci. U.S.A. 112, 106-111.

Mukhopadhyay, R., Ho, Y.-S., Swiatek, P. J., Rosen, B. P. and Bhattacharjee, H. (2006). Targeted disruption of the mouseAsna1gene results in embryonic lethality. FEBS Lett 580, 38893894.

Murata, K., Degmetich, S., Kinoshita, M. and Shimada, E. (2009). Expression of the congenital heart disease 5/tryptophan rich basic protein homologue gene during heart development in medaka fish, Oryzias latipes. Dev. Growth Differ. 51, 95-107.

Norlin, S., Parekh, V. S., Naredi, P. and Edlund, H. (2015). Asna1/TRC40 controls beta cell function and ER homeostasis by ensuring retrograde transport. Diabetes.

Okreglak, V. and Walter, P. (2014). The conserved AAA-ATPase Msp1 
confers organelle specificity to tail-anchored proteins. Proc. Natl. Acad. Sci. U.S.A. 111, 8019-8024.

Pankiv, S., Clausen, T. H., Lamark, T., Brech, A., Bruun, J.-A., Outzen, H., Øvervatn, A., Bjørkøy, G. and Johansen, T. (2007). p62/SQSTM1 binds directly to Atg8/LC3 to facilitate degradation of ubiquitinated protein aggregates by autophagy. J Biol Chem $\mathbf{2 8 2}$, 24131-24145.

Philp, L. K., Day, T. K., Butler, M. S., Laven-Law, G., Jindal, S., Hickey, T. E., Scher, H. I., Butler, L. M. and Tilley, W. D. (2016). Small Glutamine-Rich Tetratricopeptide Repeat-Containing Protein Alpha (SGTA) Ablation Limits Offspring Viability and Growth in Mice. Sci Rep 6, 28950.

Pool, M. R. (2005). Signal recognition particles in chloroplasts, bacteria, yeast and mammals (review). Mol. Membr. Biol. 22, 3-15.

Powis, K., Schrul, B., Tienson, H., Gostimskaya, I., Breker, M., High, S., Schuldiner, M., Jakob, U. and Schwappach, B. (2013a). Get3 is a holdase chaperone and moves to deposition sites for aggregated proteins when membrane targeting is blocked. J Cell Sci 126, 473483.

Powis, K., Schrul, B., Tienson, H., Gostimskaya, I., Breker, M., High, S., Schuldiner, M., Jakob, U. and Schwappach, B. (2013b). Get3 is a holdase chaperone and moves to deposition sites for aggregated proteins when membrane targeting is blocked. J Cell Sci 126, 473483.

Rabu, C., Schmid, V., Schwappach, B. and High, S. (2009). Biogenesis of tail-anchored proteins: the beginning for the end? J Cell Sci 122, 3605-3612.

Rabu, C., Wipf, P., Brodsky, J. L. and High, S. (2008). A precursorspecific role for Hsp40/Hsc70 during tail-anchored protein integration at the endoplasmic reticulum. J Biol Chem 283, 27504-27513.

Rome, M. E., Rao, M., Clemons, W. M. and Shan, S.-O. (2013). Precise timing of ATPase activation drives targeting of tail-anchored proteins. Proceedings of the ....

Rosen, B. P., Weigel, U., Karkaria, C. and Gangola, P. (1988). Molecular characterization of an anion pump. The arsA gene product is an arsenite(antimonate)-stimulated ATPase. J Biol Chem 263, 3067-3070.

Schuldiner, M., Metz, J., Schmid, V., Denic, V., Rakwalska, M., Schmitt, H. D., Schwappach, B. and Weissman, J. S. (2008). The 
GET complex mediates insertion of tail-anchored proteins into the ER membrane. Cell 134, 634-645.

Shao, S. and Hegde, R. S. (2011). Membrane protein insertion at the endoplasmic reticulum. Annu. Rev. Cell Dev. Biol. 27, 25-56.

Shao, S. and Hegde, R. S. (2016). Target Selection during Protein Quality Control. Trends Biochem. Sci. 41, 124-137.

Simpson, P. J., Schwappach, B., Dohlman, H. G. and Isaacson, R. L. (2010). Structures of Get3, Get4, and Get5 provide new models for TA membrane protein targeting. Structure 18, 897-902.

Sojka, S., Amin, N. M., Gibbs, D., Christine, K. S., Charpentier, M. S. and Conlon, F. L. (2014). Congenital heart disease protein 5 associates with CASZ1 to maintain myocardial tissue integrity. Development.

Stefanovic, S. and Hegde, R. S. (2007). Identification of a targeting factor for posttranslational membrane protein insertion into the ER. Cell 128, 1147-1159.

Stefer, S., Reitz, S., Wang, F., Wild, K., Pang, Y.-Y., Schwarz, D., Bomke, J., Hein, C., Loehr, F., Bernhard, F., et al. (2011). Structural Basis for Tail-Anchored Membrane Protein Biogenesis by the Get3Receptor Complex. Science 333, 758-762.

Subramaniam, V. N., Loh, E., Horstmann, H. and Habermann, A. (2000). Preferential association of syntaxin 8 with the early endosome. $\checkmark$ Cell Sci.

Tran, D. D., Edgar, C. E., Heckman, K. L., Sutor, S. L., Huntoon, C. J., van Deursen, J., McKean, D. L. and Bram, R. J. (2005). CAML Is a p56Lck-Interacting Protein that Is Required for Thymocyte Development. Immunity 23, 139-152.

Tran, D. D., Russell, H. R., Sutor, S. L., van Deursen, J. and Bram, R. J. (2002). CAML Is Required for Efficient EGF Receptor Recycling. Dev Cell 5, 245-256.

Vilardi, F., Lorenz, H. and Dobberstein, B. (2011). WRB is the receptor for TRC40/Asna1-mediated insertion of tail-anchored proteins into the ER membrane. J Cell Sci 124, 1301-1307.

Vilardi, F., Stephan, M., Clancy, A., Janshoff, A. and Schwappach, B. (2014). WRB and CAML Are Necessary and Sufficient to Mediate TailAnchored Protein Targeting to the ER Membrane. PLOS ONE 9, e85033.

Vogl, C., Panou, I., Yamanbaeva, G., Wichmann, C., Mangosing, S. J., 
Vilardi, F., Indzhykulian, A. A., Pangršič, T., Santarelli, R., Rodriguez-Ballesteros, M., et al. (2016). Tryptophan-rich basic protein (WRB) mediates insertion of the tail-anchored protein otoferlin and is required for hair cell exocytosis and hearing. The EMBO Journal 35, 2536-2552.

Voth, W., Schick, M., Gates, S., Li, S., Vilardi, F., Gostimskaya, I., Southworth, D. R., Schwappach, B. and Jakob, U. (2014). The Protein Targeting Factor Get3 Functions as ATP-Independent Chaperone under Oxidative Stress Conditions. Mol. Cell 56, 116-127.

Wallin, E. and Heijne, von, G. (1998). Genome-wide analysis of integral membrane proteins from eubacterial, archaean, and eukaryotic organisms. Protein Sci 7, 1029-1038.

Wang, F., Brown, E. C., Mak, G., Zhuang, J. and Denic, V. (2010). A Chaperone Cascade Sorts Proteins for Posttranslational Membrane Insertion into the Endoplasmic Reticulum. Mol. Cell 40, 159-171.

Wang, F., Chan, C., Weir, N. R. and Denic, V. (2014). The Get1/2 transmembrane complex is an endoplasmic-reticulum membrane protein insertase. Nature 512, 441-444.

Wereszczynski, J. and McCammon, J. A. (2012). Nucleotide-dependent mechanism of Get3 as elucidated from free energy calculations. Proc. Natl. Acad. Sci. U.S.A. 109, 7759-7764.

Winnefeld, M., Grewenig, A., Schnoelzer, M., Spring, H., Knoch, T. A., Gan, E. C., Rommelaere, J. and Cziepluch, C. (2006). Human SGT interacts with Bag-6/Bat-3/Scythe and cells with reduced levels of either protein display persistence of few misaligned chromosomes and mitotic arrest. Exp. Cell Res. 312, 2500-2514.

Wulff, K., Parrish, J. E., Herrmann, F. H. and Wehnert, M. (1996). Six novel mutations in the emerin gene causing $X$-linked Emery-Dreifuss muscular dystrophy. Hum Mutat 9, 526-530.

Wunderley, L., Leznicki, P., Payapilly, A. and High, S. (2014). SGTA regulates the cytosolic quality control of hydrophobic substrates. J Cell Sci 127, 4728-4739.

Yamamoto, Y. and Sakisaka, T. (2012). Molecular machinery for insertion of tail-anchored membrane proteins into the endoplasmic reticulum membrane in mammalian cells. Mol. Cell 48, 387-397.

Yofe, I., Weill, U., Meurer, M., Chuartzman, S., Zalckvar, E., Goldman, O., Ben-Dor, S., Schütze, C., Wiedemann, N., Knop, M., et al. (2016). One library to make them all: streamlining the creation of yeast libraries via a SWAp-Tag strategy. Nat Methods 13, 371-378. 
Zhao, Y., Lin, Y., Zhang, H., Mañas, A., Tang, W., Zhang, Y., Wu, D., Lin, A. and Xiang, J. (2015). Ubl4A is required for insulin-induced Akt plasma membrane translocation through promotion of Arp2/3dependent actin branching. Proc. Natl. Acad. Sci. U.S.A. 112, 96449649. 


\section{PERSONAL INFORMATION}

$\begin{array}{ll}\text { Surname, Name } & \text { Rivera Monroy, Jhon Erick } \\ \text { Date of Birth } & \text { 29 October 1984 } \\ \text { Nationality } & \text { Colombian } \\ \text { Emails } & \text { jhonriv@gmail.com } \\ & \text { jhon.riveramonroy@med.uni-goettingen.de }\end{array}$

EDUCATION

2009-2011 M.Sc. Biochemistry Universidad Nacional de Colombia, Bogotá Accumulative grade point average: 4.4/5.0

2003-2008 B.Sc. Chemistry Universidad Nacional de Colombia, Bogotá Accumulative grade point average: $\mathbf{3 . 8 / 5 . 0}$

\section{PUBLICATIONS}

- Rivera-Monroy J.*, Musiol L*. et al. (2016) Mice lacking WRB reveal differential biogenesis requirements of tail-anchored proteins in vivo. Scientific Reports. 6, 39464; doi: 10.1038/srep39464.

- Pfaff J.*, Rivera Monroy J.*, Jamieson C., Rajanala K., Vilardi F., Schwappach B., Kehlenbach R. (2016) Emery-Dreifuss muscular dystrophy mutations impair TRC40-mediated targeting of emerin to the inner nuclear membrane. J Cell Sci 129: 502516; doi: 10.1242/jcs.179333

- Castillo N., Ibáñez M., Beltrán E., Rivera-Monroy J, Ochoa J., PáezCastillo M, Posada-Buitrago M., Sulyok M, Hernández F. (2016) Identification of mycotoxins by UHPLC-QTOF MS in airborne fungi and fungi isolated from industrial paper and antique documents from the Archive of Bogotá. Environ Res, Volume 144, Part A, Pages 130-138, ISSN 0013-9351

- Spinel C., Herrera M., Rivera J. (2012) Thyroid Culture from Monolayer to Closed Follicles, Thyroid Hormone, Neeraj Kumar Agrawal (Ed.), InTech. ISBN: 978-953-51-0678-4 Available from: http://www.intechopen.com/books/thyroid-hormone/thyroid-culture-frommonolayer-to-closed-follicles

- Rivera J., Low-Calle A., Páez M., Loaiza M. (2012) El ambiente y su importancia para la conservación del patrimonio documental de la 


\section{Curriculum Vitae}

ciudad. De memoria 1: 42-47. ISSN 2322-7265

- Loaiza M., Rivera J. (2012) Identificación de microorganismos en cuero y pergamino de recursos documentales del Archivo de Bogotá. Innovación y Ciencia 19: 12-19. ISSN 0121-5140

- Herrera M., Rivera J., Curtidor H., Ondo A., Spinel C. (2009) Cultivo tridimensional sin matrix extracellular de glándula tiroides. Rev. Asoc. Col. Cienc. Biol. (Col.), 21: 73-86. ISSN 0120-4173

*Equal contribution

\section{PROFESSIONAL AND RESEARCH EXPERIENCE}

11.2013 to 05.2017

01.2011 to 09.2013

02.2009 to 10.2011
PhD project, Department of Molecular Biology, University Medicine Göttingen.

Prof. Dr. Blanche Schwappach

- Role of WRB protein in cardiac function.

Researcher at Laboratory of Chemistry and Biology, Archive of Bogotá.

Supervisors: Dr. Martha Lucía Posada.

- Detection of mycotoxigenic and cellulolytic fungi in documents and related indoor environments of public archives of Bogotá (Colombia).

- Molecular identification of filamentous fungi isolated from documentary material of the Archive of Bogota (Colombia).

Master in Science Biochemistry, Laboratory of Biophysics, International Physics Center-Universidad Nacional de Colombia.

Prof. Dr. Clara Spinel

- Morpho-functional analysis of rat and pig isolated thyroid follicles under iodide and thyrotropin effects.

09.2007 to Diploma thesis. Laboratory of Biophysics, International 09.2008
Physics Center-Universidad Nacional de Colombia

Prof. Dr. Clara Spinel

- lodide uptake and organification in pig Thyroid tridimensional cultures under thyrotropin effect. 
Curriculum Vitae

\section{SCHOLARSHIPS AND AWARDS}

People Progamme -Marie Curie Actions of the European Union's Seventh Framework Programme (FP7/20072013/)

Young Scientists and Innovators "Virginia

Gutiérrez de Pineda"

Scholarship (2009)

Exemption of academic fees

National Award in Basic Medical Science (October 2008)
Early-stage research fellowship given to the work "Role of WRB in cardiac function (PhD project)".

This scholarship was given to the work "Functional study of human thyroid follicle cultures that reproduce in vivo function" by the Administrative Department of Science, Technology and Innovation COLCIENCIAS.

During my master's degree studies, I was exempted of academic fees in three occasions by the School of Sciences of the National University of Colombia.

This award was given to the work "Threedimensional culture of thyroid gland without extracellular matrix" by the Colombian Association of Biological Science. 